\title{
Physical aspects of ferroelectric semiconductors for photovoltaic solar energy conversion
}

\author{
Pilar Lopez-Varo, ${ }^{1}$ Luca Bertoluzzi, ${ }^{2}$ Juan Bisquert, ${ }^{* 2,3}$ Marin Alexe, ${ }^{4}$ Mariona \\ Coll $^{5}$, Jinsong Huang, ${ }^{6}$ Juan Antonio Jimenez-Tejada, ${ }^{1}$ Thomas Kirchartz, ${ }^{7,8}$ Riad \\ Nechache, ${ }^{9}$ Federico Rosei, ${ }^{9}$ Yongbo Yuan ${ }^{6}$
}

${ }^{1}$ Departamento de Electrónica y Tecnología de Computadores, CITIC-UGR, Universidad de Granada, 18071 Granada, Spain

${ }^{2}$ Institute of Advanced Materials (INAM), Universitat Jaume I, 12006 Castelló, Spain

${ }^{3}$ Department of Chemistry, Faculty of Science, King Abdulaziz University, Jeddah, Saudi Arabia

${ }^{4}$ Department of Physics, University of Warwick, Coventry CV4 7AL, UK.

${ }^{5}$ Institut de Ciència de Materials de Barcelona (ICMAB-CSIC), Campus UAB, 08193, Bellaterra, Catalonia, Spain

${ }^{6}$ Department of Mechanical and Materials Engineering and Nebraska Center for Materials and Nanoscience, University of Nebraska-Lincoln, Lincoln, Nebraska 685880656, USA

${ }^{7}$ IEK5-Photovoltaik, Forschungszentrum Jülich, 52425 Jülich, Germany

${ }^{8}$ Faculty of Engineering and CENIDE, University of Duisburg-Essen, Carl-Benz-Str. 199, 47057 Duisburg, Germany

${ }^{9}$ INRS - Centre Énergie, Matériaux et Télécommunications, Boulevard Lionel-Boulet, Varennes, Québec, J3X 1S2, Canada

Email: bisquert@uji.es

20 July 2016

\section{Content}

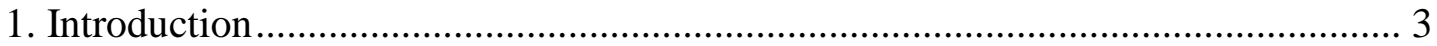

2. Photovoltaic mechanisms in conventional solar cells.......................................... 7

3. Phenomenology of ferroelectric solar cells......................................................... 19

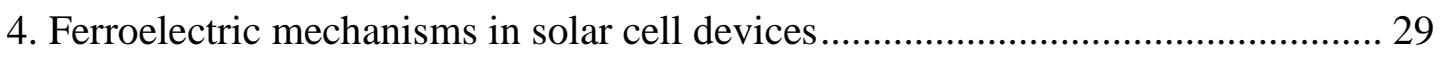

4.1. Change of injection barriers and depolarization field ................................. 30

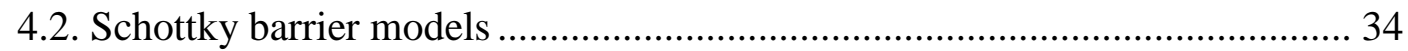

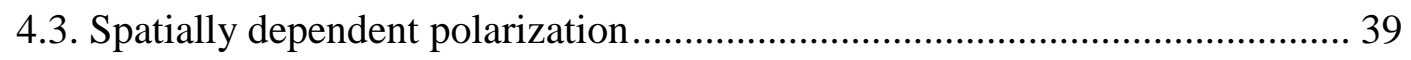

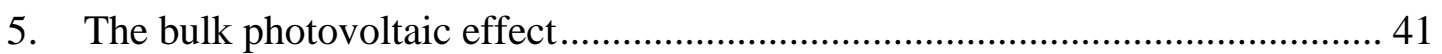

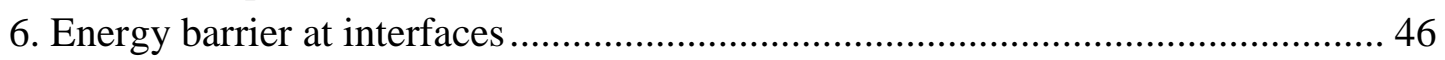

7. Advanced materials concepts in ferroic photovolatics ................................... 52

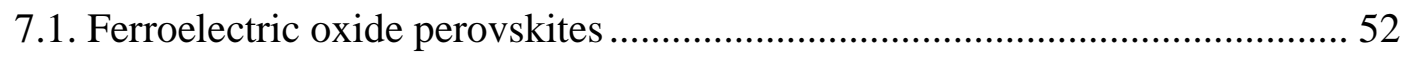


7.2. Multiferroic materials 54

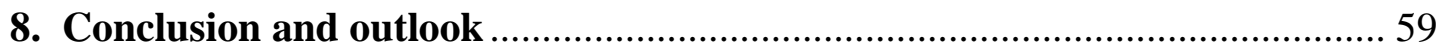

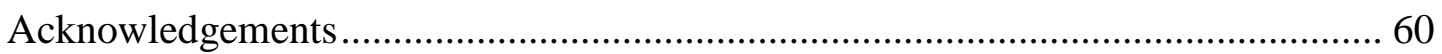

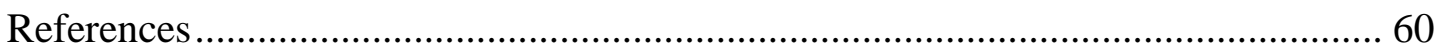

\section{Abstract}

Solar energy conversion using semiconductors to fabricate photovoltaic devices relies on efficient light absorption, charge separation of electron-hole pair carriers or excitons, and fast transport and charge extraction to counter recombination processes. Ferroelectric materials are able to host a permanent electrical polarization which provides control over electrical field distribution in bulk and interfacial regions. In this review, we provide a critical overview of the physical principles and mechanisms of solar energy conversion using ferroelectric semiconductors and contact layers, as well as the main achievements reported so far. In a ferroelectric semiconductor film with ideal contacts, the polarization charge would be totally screened by the metal layers and no charge collection field would exist. However, real materials show a depolarization field, smooth termination of polarization, and interfacial energy barriers that do provide the control of interface and bulk electric field by switchable spontaneous polarization. We explore different phenomena as the polarization-modulated Schottky-like barriers at metal/ferroelectric interfaces, depolarization fields, vacancy migration, and the switchable rectifying behavior of ferroelectric thin films. Using a basic physical model of a solar cell, our analysis provides a general picture of the influence of ferroelectric effects on the actual power conversion efficiency of the solar cell device, and we are able to assess whether these effects or their combinations are beneficial or counterproductive. We describe in detail the bulk photovoltaic effect and the contact layers that modify the built-in field and the charge injection and separation in bulk heterojunction organic cells as well as in photocatalytic and water splitting devices. We also review the dominant families of ferroelectric materials that have been most extensively investigated and have provided the best photovoltaic performance. 


\section{Introduction}

The recent decades have witnessed a large surge of research on solar conversion technologies. Photovoltaics (PV) is considered a most promising renewable energy technology for the coming decades, but substantial improvements are yet required to improve efficiencies and reduce the fabrication costs of present day materials. Despite the rapid increase in the PV performance of ferroelectric based solar cells, substantial efforts to explore optimal device configuration and maximize carrier generation and collection are still required to deploy commercial technologies. PV markets are currently dominated by solar cells based on crystalline silicon, with highest efficiencies of about $18 \%$ in single junction devices. This technology is well established, however its comparatively high cost with respect to carbon fossil fuel based energies still limits further development. This is mainly due to the high cost of crystalline silicon and to additional complex and expensive fabrication and processing steps. Several efforts are being undertaken to address these issues, in particular to (i) decrease the cost of silicon fabrication, (ii) increase cell efficiency and (iii) develop new generations of solar cells based on alternative materials. There is still a need for solar technologies leading to high conversion efficiencies by using simplified components, architectures, fabrication and processing.

Ferroelectricity is an appealing property for PV applications as it allows one to control the internal electric fields and injection barriers, which lie at the heart of the PV mechanism. When ferroelectric materials are sandwiched between electrodes and illuminated, they can exhibit a variety of rich phenomena including the generation of photo-excited charge carriers. The ferroelectric layer provides a polarization-induced internal electric field which can help in separating the photo-excited carriers, generating effective built-in fields at the ferroelectric/electrode interface due to Schottky barriers that can deplete the ferroelectric layers, etc. In addition, the ferroelectric materials give rise to unique PV behavior based on atomistic asymmetry of current generation, the bulk PV effect. The ferroelectric oxides are an intriguing class of photovoltaic materials, known to produce a very high photovoltage, up to orders of magnitude larger than the bandgap, but rather a small photocurrent. Thus power conversion efficiencies that have been orders of magnitude smaller than for classical photovoltaic devices for many decades. However, in recent years there has been a significant enhancement in efficiencies, as indicated in Fig. 1. The most significant advance has been obtained with $\mathrm{Bi}_{2} \mathrm{FeCrO}_{6}$ (BFCO), which is a multiferroic material, as proposed by Spaldin and coworkers (Baettig et al., 2005,Baettig and Spaldin, 2005), that exhibits both a small bandgap and a large photocurrent density $\left(11.7 \mathrm{~mA} \mathrm{~cm}^{-2}\right)$, yielding a power-conversionefficiency (PCE) as high as 3.3\% in single layers (Nechache et al., 2015). By controlling the $\mathrm{Fe} / \mathrm{Cr}$ cationic order and their distribution in the films, it was reported that the band gap of BFCO can be tuned in the range of 2.1 to $1.4 \mathrm{eV}$. This led to the design of a multi-absorber system based on BFCO, obtained by stacking three layers with different bandgaps, leading to a breakthrough solar cell with a PCE of $8.1 \%$, where the normal 
PV effect has been aided by the ferroelectric property to create a better barrier.

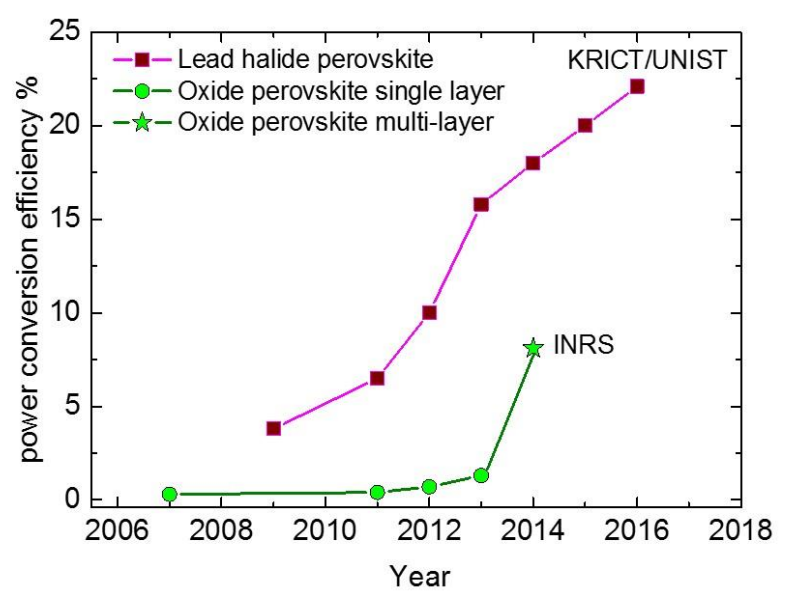

Fig. 1. Evolution of top energy conversion efficiency of perovskite solar cells.

Building on this success, lower cost materials and device fabrication approaches for high efficiency ferroelectric PV should be developed for practical and reliable applications. Attaining this objective requires an in depth understanding of the operation mechanism of the ferroelectric PV effect in such materials, which in turn will require close collaboration between experiment and theory. Depending on the response mechanism that is triggered in ferroelectric layers, they may be used for potential applications in photovoltaics, high resolution imaging, sensing and many more functional technologies. A variety of interesting phenomena arise from the photoinduced effects in ferroelectrics, for example, the deformation induced by irradiation of light which can be explained as the combination of photovoltaic and piezoelectric effects. The better understanding of these mechanisms should be in the focus of the community in order to enhance the performance of ferroelectric solar cells. Nowadays, there is still an intense debate regarding the mechanism which underpins the photovoltaic effect observed in ferroelectric materials and how to improve the power conversion efficiency. The main objective of this review is to provide a broad assessment of the current situation in this growing area of research.

Ferroelectricity is characterized by permanent electric dipoles in the material that remain in the absence of an applied electric field. In contrast to ordinary dipolar polarization, the material is always in a polarized state in which a remnant polarization $P_{r}$ exists even if the applied electric field $F$ is removed. Furthermore, the polarization direction can be reoriented (switched) by an applied field larger than a certain value which is denoted coercive field, $F_{c}$. Due to symmetry reasons, a certain degree of crystallinity is required for materials to display a ferroelectric behavior. Simple perovskites are a broad class of materials considered for PV with a structure $\mathrm{ABO}_{3}$, where $\mathrm{A}$ and $\mathrm{B}$ are cations and $\mathrm{O}$ anions as oxygen, nitrogen or trihalides. In some of 
these systems, polarization arises from the relative displacements of ions that provide a stable configuration, see the displacements calculated in Fig. 2a, and the correspondent value of polarization, $P_{s}$, the latter being dependent not only on the existing electric field but also on its history, yielding a hysteresis loop, as can be observed in Fig. 2b. Typically, ferroelectric materials undergo a phase transition from a high symmetry phase, where no spontaneous polarization may exist, to a lower symmetry phase below the Curie temperature. In addition to ionic displacement and distortions that cause the ferroelectric effect, the modern theory of polarization based on quantum calculation indicates subtle effects of electron-electron interactions and/or coupling of electronic and ionic effects. For example, ferroelectricity in $\mathrm{BiFeO}_{3}$ originates from the dynamic $\mathrm{O}$ $p$-Bi $p$ hybridization (Neaton et al., 2005,Clark and Robertson, 2007) (Lines and Glass, 2001).
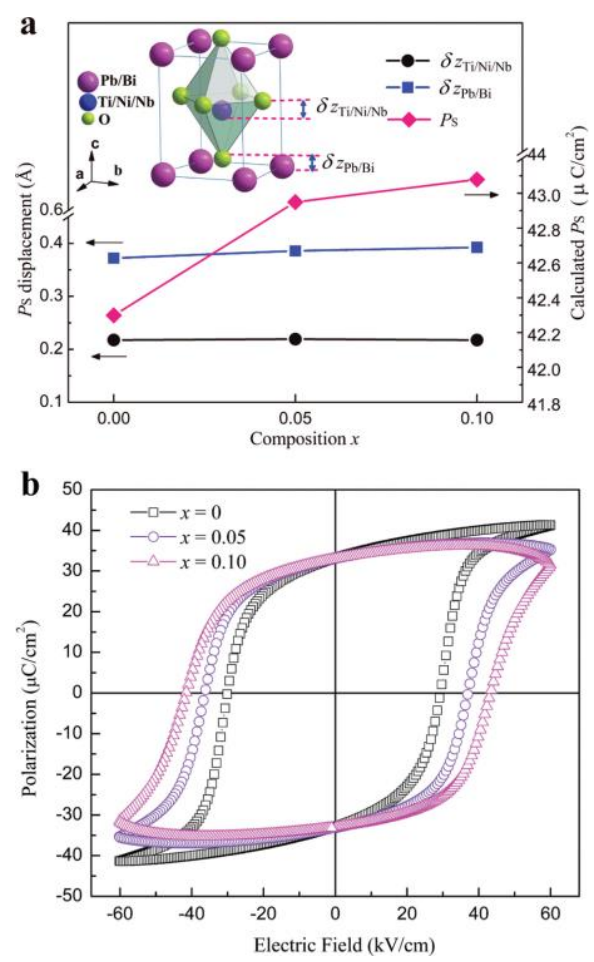

Figure 2. The figure shows a generic behaviour of a ferroelectric oxide perovskite with a general formula $\mathrm{ABO}_{3}$. (a) The displacement and the calculated $P_{s}$ of 0.65 $\mathrm{PbTiO}_{3}-0.35 \mathrm{Bi}\left(\mathrm{Ni} 2 / 3+\mathrm{x} \mathrm{Nb}_{1 / 3-\mathrm{x}}\right) \mathrm{O}_{3-\delta}$ as function of $\mathrm{x}$. The inset displays the spontaneous polarization displacement at $\mathrm{A}$ and $\mathrm{B}$ sites in the perovskite lattice. (b) Hysteresis loops of $0.65 \mathrm{PbTiO}_{3}-0.35 \mathrm{Bi}\left(\mathrm{Ni}_{2 / 3+\mathrm{x}} \mathrm{Nb}_{1 / 3-\mathrm{x}}\right) \mathrm{O}_{3-\delta}(\mathrm{x}=0,0.05$, and 0.10$)$ ceramics measured at a frequency of $1 \mathrm{~Hz}$. Reprinted from (Liu et al., 2015).

From a theoretical point of view, the ferroelectric polarization affects the transport behavior in semiconductors by means of changes in the band bending and electrical field effects (Batra et al., 1973,Blom et al., 1994,Qin et al., 2009,Suryanarayana and Bhattacharya, 2012). It has been observed in experimental and numerical works (Ge et 
al., 2011,Wang et al., 2011,Yi et al., 2011) that polarization-modulated Schottky-like barriers at metal/ferroelectric interfaces produce a switchable rectifying behavior in a ferroelectric thin film. Thus, controlling the polarization with an external field allows electrical tuning of charge transport and hence achieving unidirectional electric conduction. Other phenomena relevant to ferroelectric solar cells that will also be considered in are the depolarization field, the bulk photovoltaic current, ion migration or domain walls.

The majority of the studies on ferroelectric photovoltaics has been centered on a restricted set of oxide perovskites and their variational compositions: $\mathrm{LiNbO}_{3}$ (LNO), $\mathrm{BaTiO}_{3}$ (BTO), $\mathrm{Pb}(\mathrm{Zr}, \mathrm{Ti}) \mathrm{O}_{3}\left(\mathrm{PZT}\right.$ ), and $\mathrm{BiFeO}_{3}$ (BFO) (Fan et al., 2015) (Jalaja and Dutta, 2015). A characteristic configuration is obtained by growing samples of epitaxial quality on a $\mathrm{SrRuO}_{3}$ layer (SRO) or $\mathrm{SrTiO}_{3}$ (STO) substrate, and completing the device with a sputtered semitransparent layer of a metal such as Pt. Another route of investigation is to introduce ferroelectric effects in organic photovoltaic devices (OPV). The main organic ferroelectric materials are PVDF [polyvinylidene fluoride; $\left(\mathrm{CH}_{2} \mathrm{CF}_{2}\right) \mathrm{n}$ ] and its copolymer P(VDF-TrFE) [poly(vinylidene fluoridetrifluoroethylene); $\left.\left(\mathrm{CH}_{2} \mathrm{CF}_{2}\right) n-\left(\mathrm{CHFCF}_{2}\right)_{\mathrm{m}}\right]$ due to easy processability and stability (Naber et al., 2010). These have been applied in organic solar cells, in order to enhance charge collection and charge separation (Yang et al., 2012) (Yuan et al., 2011).

Organic-inorganic perovskite thin films of $\mathrm{CH}_{3} \mathrm{NH}_{3} \mathrm{PbI}_{3}$, containing an organic cation such as methylammonium $\left(\mathrm{MA}^{+}\right)$in between octahedral cages formed by $\mathrm{Pb}^{2+}$ and a halide cation anion such as $\mathrm{I}^{-}$, have formed a new class of low cost and highly efficient PV devices, in a few years of research, starting with the report of (Kojima et al., 2009), see Fig. 1. Although a considerable debate exists in the literature about the ferroelectric property of $\mathrm{MAPbI}_{3}$ and related perovskites, recent studies indicated that they are not ferroelectric at room temperature (Coll et al., 2015,Fan et al., 2015). First of all it needs to be pointed out that several phenomena like the large electronic conduction current and substantial ionic conduction and polarization of these materials make the determination of ferroelectric features problematic. (Coll et al., 2015) utilized piezoresponse force microscopy (PFM) to show that the polarization in the lead halide perovskite thin film vanishes in a time scale of seconds. The main mechanism of polarization consists on the orientational polarization of $\mathrm{MA}^{+}$cation (which has significant dipole moment). The weak ferroelectricity of $\mathrm{MAPbI}_{3}$ is attributed to the fact that the $\mathrm{MA}^{+}$the molecule is fully activated and free to rotate at room temperature (Poglitsch and Weber, 1987,Onoda-Yamamuro et al., 1990,Mosconi et al., 2014,Fan et al., 2015,Filippetti et al., 2015,Leguy et al., 2015,Mattoni et al., 2015). The ferroelectric ordering is effective only when the barrier for rotation of MA becomes significant, below $160 \mathrm{~K}$, when the transition from tetragonal to orthorhombic phases occur. As the hybrid and organic perovskites have been most extensively reviewed in the literature they will not be further treated in the present report.

Several excellent review articles have been presented in recent years on ferroelectric 
photovoltaics (Sheu et al., 2012,Yuan et al., 2014,Butler et al., 2015,Fan et al., 2015) (Akbashev et al., 2016,Paillard et al., 2016) and on the physical properties of ferroelectric materials (Naber et al., 2010,Pintilie, 2011) that updated the early treatises (Fridkin, 1979,Belinicher, 1980,Ruppel et al., 1982,Sturman and Fridkin, 1992). The present work aims at taking a new stance of analysis of the state of the art by examining the specific physical mechanisms and device properties that determine the photovoltaic response of the solar cells based on ferroelectric materials. Including ferroelectric effects in solar cells introduces a number of significant effects, as the ferroelectric polarization strongly affects the processes that regulate photovoltaic operation. The electrical current and voltage generated in ferroelectric solar cells has in fact two origins (Ruppel et al., 1982). The first one is the conventional photovoltaic effect (sometimes called barrier photovoltaic effect), as in classical non-ferroelectric solar cells. It is due to an asymmetry in the real space, but it may be influenced by polarization charges. The second effect is the bulk photovoltaic effect which arises from asymmetries in k-space. As both effects have been amply studied in recent years, we devote a deep study to this topic divided into several sections. For the sake of clarity, we provide in Section 2 a detailed discussion of the classical solar cells, based on the mechanisms of charge separation and charge collection, which can be obtained with normal non ferroelectric semiconductors. Then, in Section 3, we discuss the actual phenomenology of ferroelectric solar cells, including the typical response of the solar cell, as well as the best results obtained so far in terms of performance. In Section 4, we develop a systematic evaluation of the different photovoltaic mechanisms that are influenced by ferroelectric polarization, and we discuss systematic simulations based on numerical modeling to illustrate the effects of these mechanisms. Section 5 is dedicated to discussing the bulk photovoltaic effect. In Section 6, we review another aspect of ferroelectric layers, which can be used to modify the built-in field or the injection properties when inserted into a photoactive structure, in order to improve the photovoltaic performance. In Section 7, a brief overview of the main types of ferroelectrics perovskites for solar energy conversion is presented. Finally, perspectives for future works are presented in Section 8.

\section{Photovoltaic mechanisms in conventional solar cells}

The photovoltaic properties have established many decades ago following the growth of crystalline semiconductors solar cells (Sze, 1981,Bube, 1992). However, in recent years a much broader class of materials and devices was studied and discussed, including all-inorganic, hybrid organic/inorganic, planar and nanostructured solar cells, thin film inorganic technologies, dye-sensitized solar cells, organic solar cells, and very recently hybrid organic-inorganic perovskite solar cells. A number of general PV operation principles and general properties of solar cells have been established (Bisquert et al., 2004,Würfel, 2005,Bisquert and Garcia-Belmonte, 2011,Kirchartz and Rau, 2011,Kirchartz et al., 2015) that will be summarized here for later comparison with the 
ferroelectric photovoltaic devices.

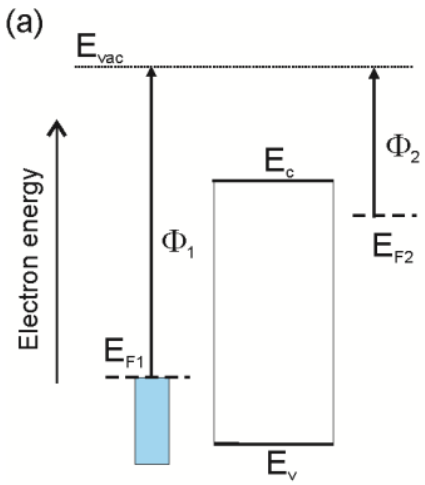

(b)
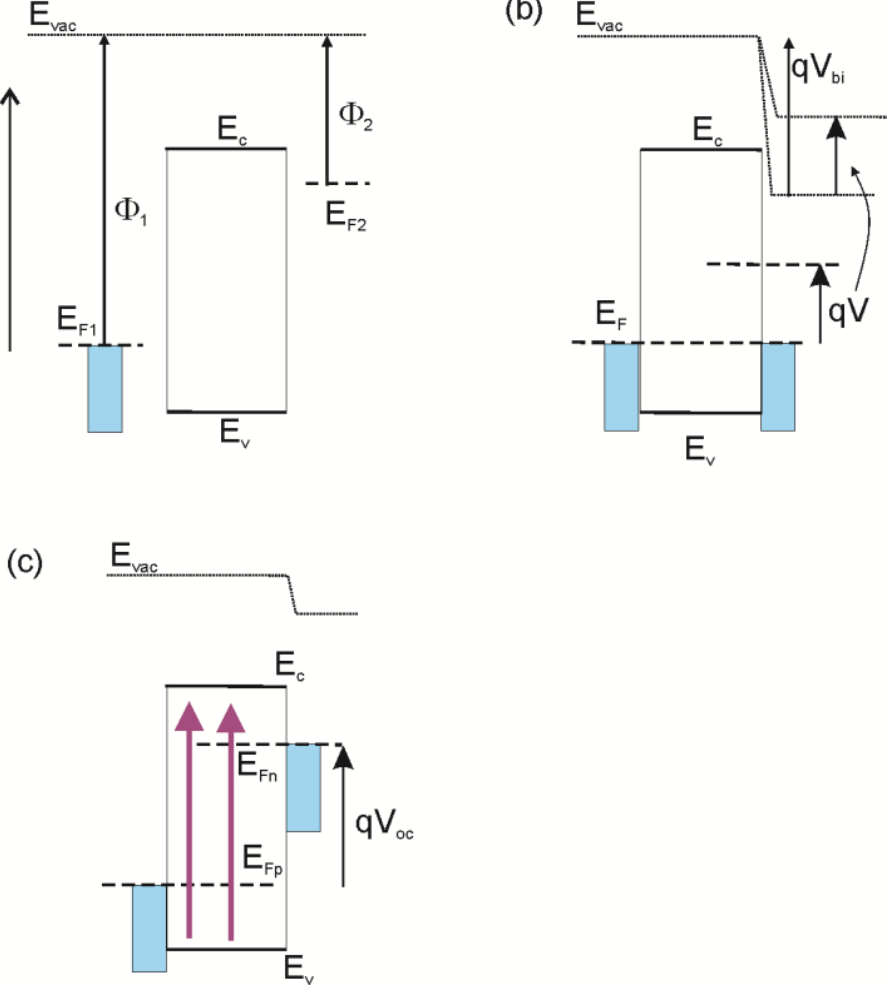

Fig. 3. Formation of an elemental diode-like device. (a) Separate materials, a semiconductor with conduction band edge minimum $E_{c}$ and valence band maximum $E_{v}$, and two contacts with work function $\Phi_{1}$ and $\Phi_{2}$. (b) Equilibration of Fermi levels for the materials in contact, by formation of a surface dipole indicated in the vacuum level (VL). The situation of an applied voltage $V$ is also shown. (c) Under photogeneration, the diode is a solar cell that produces a photovoltage.

In Fig. 3a, we represent a layer of a light absorbing semiconductor with valence and conduction band edges, $E_{v}$ and $E_{c}$, respectively, that define the semiconductor bandgap $E_{g}=E_{c}-E_{v}$. To produce electricity, the optically active semiconductor layer needs to have suitable contacts. An asymmetry needs to be built in the device that will turn the process of generation of electrons and holes (which is completely symmetric in normal semiconductors) into a photovoltage and photocurrent. This asymmetry is realized by using contacts of different work functions, in which "contact" has a rather wide meaning, as it could be, for example, a redox electrolyte in a dye-sensitized solar cell (DSC). To simplify the present matter, we show in Fig. 3a a simple planar geometry, in which the semiconductor and metals with work function $\Phi_{1}$ and $\Phi_{2}$ are not yet in contact. In Fig. 3b, equilibration leads to a common Fermi level $E_{F}$, that requires to accommodate the original difference of work functions that corresponds to the built-in potential $V_{b i}$ 


$$
V_{b i}=\frac{\Phi_{1}-\Phi_{2}}{q}
$$

in terms of positive elementary charge $q$.

In general, a metal or metal oxide contact with low work function $\Phi_{2}$ predominantly equilibrates electronically to the conduction band level $E_{c}$, and is suited to extract electrons, while metals, metal oxides or organic conductors with a larger work function, $\Phi_{1}$, are better suited to establish equilibrium with the valence band edge levels for the transference of holes, as indicated in Fig. 3b. Therefore the energetic alignment is a very important property for contacts in high efficiency solar cells. For example, in $\mathrm{MAPbI}_{3}$ perovskite solar cell, a very common contact combination is shown in Fig. 4. On the one hand, a metal oxide such as $\mathrm{TiO}_{2}$ is well aligned to accept electrons from the conduction band of the perovskite absorber. On the other hand, the hole transport material (HTM) spiro-OMeTAD shows a good alignment to the valence band level. Due to the properties of the two contacts, the deleterious processes (4) and (5) of Fig. 4 occur to a minimal extent and the solar cell produces a large photocurrent.

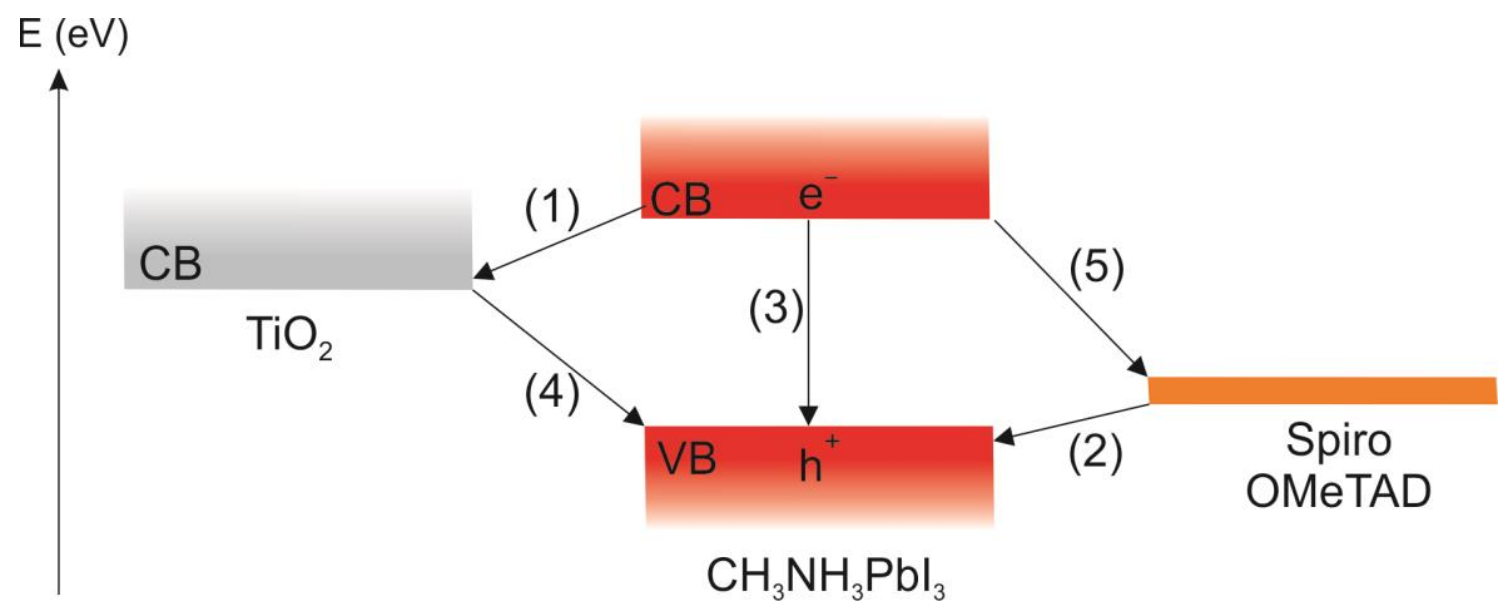

Fig. 4. Schematic diagram of energy levels and electron transfer processes in an $\mathrm{HTM} \mid$ perovskite $\mid \mathrm{TiO}_{2}$ cell. The perovskite absorbs light and electron-hole pairs are created in the semiconductor, then the photogenerated (1) electron and (2) hole are injected from the perovskite into $\mathrm{TiO}_{2}$ and hole-transporting material (HTM), respectively. (4) The electron-hole pair could be annihilated by recombination. Other recombination steps are: (5) back electron transfer at the $\mathrm{TiO}_{2}$ surface to the perovskite, (6) back charge transfer at the HTM surface. Finally, all these steps are competed with the extraction of the photogenerated charges. Adapted from (Marchioro et al., 2014).

The electric field is related to the electrostatic potential distribution along a device $\phi(x)$ as

$$
F=-\frac{d \phi}{d x}
$$

The voltage $V$, externally applied to the device, creates a separation of the Fermi 
levels of the contacting materials (Bisquert, 2014). If $d$ is the thickness of the semiconductor and if the cathode is at $x=0$ and the anode at $x=d$, we have

$$
q V=E_{F n}(0)-E_{F p}(d)
$$

In Fig. 3b, the original work function difference is accommodated as a surface dipole at the electron extracting contact (cathode) (Guerrero et al., 2012), as indicated by the tilt of the vacuum level (VL) which shows where the internal field is located. The application of a voltage, as indicated in Fig 3b, rises the minority-carrier (electron) Fermi level, $E_{F n}$, while the hole Fermi level, $E_{F p}$, remains stationary. Consequently, the surface dipole is reduced, as this is where the applied voltage drops. Fig. 3c shows the cell under illumination at open circuit. Above-bandgap absorption promotes the creation of electron hole pairs. The microscopic separation of generated carrier pairs creates an excess of electrons in the conduction band. The creation of minority carriers therefore produces a significant displacement of their Fermi level $E_{F n}$, which yields the open-circuit photovoltage $V_{o c}$.

(a)

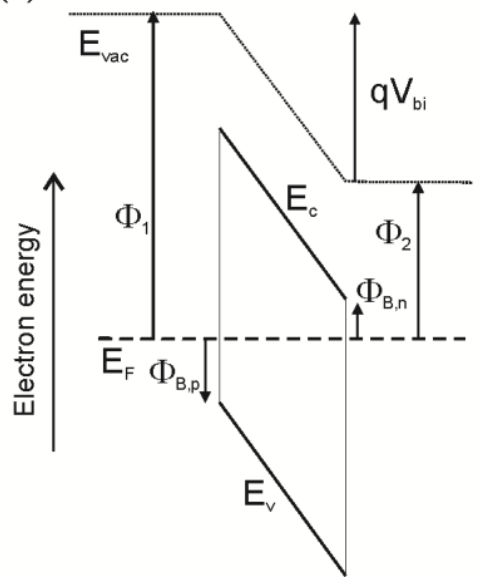

(b)

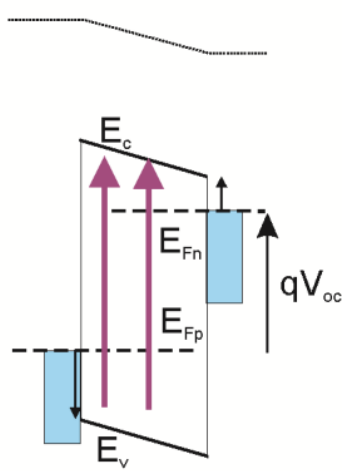

Fig. 5. Energy diagram for a MIM after contact of electrode materials, showing the built-in voltage given by the difference in work functions between both metals $\left(V_{b i}=\left(\Phi_{1}-\Phi_{2}\right) / q\right)$. (b) Photovoltage under illumination. The Fermi levels are flat, and for each carrier the diffusion current equilibrates the drift current.

While the example of Fig. 3b is a useful photovoltaic model, there are other types of electric field distribution that are also significant. In Fig. 5a, we show a different type of equilibration, which corresponds to a metal-insulator-metal (MIM) structure. The energy barrier for electron injection is defined as the difference between the energy level in the semiconductor and the metal, namely $\Phi_{B, n}=E_{c}-E_{F 2}$, and the barrier for hole injection is $\Phi_{B, p}=E_{v}-E_{F 1}$. In the model of Fig. 5a, the initial injection barriers of Fig. 3a are considered fixed, so that no surface dipole is available for the Fermi levels to align. Therefore, the built-in voltage is distributed along the whole absorber layer, in 
which the drift field has the value:

$$
F=\frac{1}{d}\left(V_{b i}-V\right)
$$

Eq. (2) and (4) indicate that an applied voltage also corresponds to the variation of VL across the full device thickness (Bisquert, 2014). Under illumination, the drift field will be reduced as the Fermi levels of electrons and holes are separated further away from their equilibrium position.

The displacement vector is a fundamental quantity for the description of the semiconductor electrostatics. Such vector contains contributions from the electric field and the polarization vector $P$. The dielectric displacement vector is given by:

$$
D=\varepsilon_{0} F+P
$$

where $\varepsilon_{0}$ is the vacuum permittivity. The dielectric displacement is related to the free space charge density as

$$
\frac{d D}{d x}=\rho_{f}
$$

Combining (5) and (6) results in

$$
\begin{aligned}
& \frac{d D}{d x}=\varepsilon_{0} \frac{d F}{d x}+\frac{d P}{d x} \\
& \frac{d F}{d x}=\frac{1}{\varepsilon_{0}} \frac{d D}{d x}-\frac{1}{\varepsilon_{0}} \frac{d P}{d x}=\frac{1}{\varepsilon_{0}}\left(\rho_{f}+\rho_{p o l}\right)=\frac{\rho_{T}}{\varepsilon_{0}}
\end{aligned}
$$

where polarization charge density is

$$
\rho_{p o l}=-\frac{d P}{d x}
$$

and $\rho_{T}=\rho_{f}+\rho_{p o l}$. For the particular case of a semiconductor with space charges, then Eq.(8) would read

$$
\frac{d F}{d x}=\frac{\rho_{T}}{\varepsilon_{0}}=\frac{\rho_{f}+\rho_{p o l}}{\varepsilon_{0}}=\frac{q}{\varepsilon_{0}}\left(p-n-N_{A}^{-}+N_{D}^{+}+\rho_{p o l}\right)
$$

where the space-charge density, $\rho_{f}=q\left(n-p-N_{A}^{-}+N_{D}^{+}\right)$, includes the free electron, $n$, and hole $p$ densities, the fixed ionized donor density ${N_{D}}^{+}$and the acceptor density $N_{A}{ }^{-}$. The total charge density across the semiconductor, $\rho_{T}$, also includes the $\rho_{\text {pol }}$.

For the general treatment of linear non-ferroelectric dielectrics and ferroelectric materials, the relative dielectric permittivity is defined as

$$
\varepsilon_{r} \equiv \frac{1}{\varepsilon_{0}} \frac{d D}{d F}
$$

Combining Eq. (11) $\left(\mathrm{d} D / \mathrm{d} x=\varepsilon_{0} \varepsilon_{\mathrm{r}} \mathrm{d} F / \mathrm{d} x\right)$, with Eqs. (7) and (9), the polarization charge density is related to the free space-charge density as 


$$
\rho_{p o l}=-\frac{d P}{d x}=-\left(\frac{d D}{d x}-\varepsilon_{0} \frac{d F}{d x}\right)=-\left(\varepsilon_{r}-1\right)\left(\rho_{p o l}+\rho_{f}\right)=-\frac{\varepsilon_{r}-1}{\varepsilon_{r}} \rho_{f}
$$

From Eq. (12), Eq. (6) can be written as:

$$
\frac{d F}{d x}=\frac{\rho_{f}}{\varepsilon_{0} \varepsilon_{r}(P, F)}
$$

where the relative permittivity $\varepsilon_{r}$ is given by the slope of the polarization curve in the linear regime, beyond the coercive field (Eqs. (5) and (11)), as:

$$
\varepsilon_{r}(P, F)=1+\frac{1}{\varepsilon_{0}} \frac{d P}{d F}
$$

In a linear non ferroelectric dielectric, the dielectric polarization is proportional to the electric field $F$ and $\varepsilon_{r}$ is a constant, which can be defined as the effective relative permittivity in absence of spontaneous polarization. Thus,

$$
P(F)=\varepsilon_{0}\left(\varepsilon_{r}-1\right) F
$$

and

$$
D=\varepsilon_{0} \varepsilon_{r} F
$$

In a ferroelectric material, $P$ is not linear with $F$, exhibiting the typical hysteresis cycle displayed in Fig. $2 \mathrm{~b}$ and in Fig. S3. For these materials, the relative permittivity follows the general expression (14). Depending on the models used for the $P-F$ relation, different effects can be described for a ferroelectric sandwiched between two metal contacts, which will be discussed in detail in subsequent sections. In some cases, the $P-F$ hysteresis cycle is modeled by the switching between two constant values for the polarization when the coercive fields are surpassed. This means that the polarization is spatially independent along the ferroelectric polarization direction with a discontinuity at the contacts. This situation creates polarization-induced surface charges of opposite sign at the contact with the electrodes. Other models consider that the polarization is electric-field dependent, as shown in Fig. 2b, even at values different from the coercive fields. In this case, the polarization is spatially dependent giving rise to the surface polarization charges plus a non-negligible distribution of polarization charges within the ferroelectric bulk.

In addition to the previous electrostatic features, the model of a solar cell requires the knowledge of the charge carrier transport, through the current densities of electrons and holes $J_{\mathrm{n}, \mathrm{p}}$, which include the drift and diffusion components:

$$
\begin{aligned}
& J_{n}=q n \mu_{n} F+q D_{n} \frac{d n}{d x} \\
& J_{p}=q p \mu_{p} F-q D_{p} \frac{d p}{d x}
\end{aligned}
$$

Here $\mu_{\mathrm{n}}$ and $\mu_{\mathrm{p}}$ are the charge carrier mobilities and $D_{\mathrm{n}}$ and $D_{\mathrm{p}}$ are the diffusion 
coefficients, for electrons and holes, respectively. The diffusion coefficients and the mobilities are connected via the Einstein relation $D_{\mathrm{n}} / \mu_{\mathrm{n}}=D_{\mathrm{p}} / \mu_{\mathrm{p}}=k_{\mathrm{B}} T / \mathrm{q}$, where $k_{\mathrm{B}}$ is the Boltzmann constant and $T$ is the absolute temperature. Electron and hole conductivities are defined as $\sigma_{n}=q n \mu_{n}, \sigma_{p}=q p \mu_{p}$. In general the total current density of each type of carrier is directly related to the gradient of their Fermi level as follows

$$
\begin{aligned}
& J_{n}=\frac{\sigma_{n}}{q} \nabla E_{F n} \\
& J_{p}=\frac{\sigma_{p}}{q} \nabla E_{F p}
\end{aligned}
$$

Total current is

$$
J=J_{n}+J_{p}
$$

Finally, the continuity equations relate the electron and hole current densities to the charge carrier generation and recombination rates, $G(\mathrm{x})$ and $R(\mathrm{x})$, respectively:

$$
\begin{aligned}
& \frac{d J_{n}}{d x}=-q[G(x)-R(x)] \\
& \frac{d J_{p}}{d x}=q[G(x)-R(x)]
\end{aligned}
$$

Different expressions for the boundary conditions related to selective contacts are described in SI.

The main figure of merit of a solar cell for the purpose of solar energy conversion is the current density-voltage $(J-V)$ characteristic. From the $J-V$ curve, one can extract an important parameter, the solar to electric power conversion efficiency (PCE, also denoted $\eta$ ) which is the ratio between the maximum generated electrical power and the incident power $\left(P_{i n}\right)$. The maximum power point $(\mathrm{mp})$ is usually written in term of the open-circuit voltage $\left(V_{o c}=V(J=0)\right)$, the short-circuit current density $\left(J_{s c}=J(V=0)\right)$, and the fill factor $(\mathrm{FF})$ :

$$
\eta=\frac{J_{s c} V_{o c} F F}{P_{i n}}
$$

where, FF is related to the voltage and current density of maximum power generation as:

$$
F F=\frac{J_{m p} V_{m p}}{J_{s c} V_{o c}}
$$

The standard reference for illumination is AM1.5G that simulates solar spectral irradiation with an integrated power of $1000 \mathrm{~W} \mathrm{~m}^{-2}=100 \mathrm{~mW} \mathrm{~cm}^{-2}$ (one sun intensity). Due to the very low efficiency of ferroelectric solar cells, normally the detailed spectral characteristics of incident light are not reported and simply it is labeled as monochromatic, white light or UV light.

The $J-V$ characteristic for a particular type of solar cell can be predicted using the 
above described equations. We first describe a rather simple but important model that can be derived from Fig. 3. A balance between charge generation, recombination and extraction is established (Bisquert and Garcia-Belmonte, 2011)

$$
J=J_{s c}-J_{r e c}(V)+J_{0}
$$

Here $J_{0}$ is a thermal carrier generation term called the dark saturation current density that ensures equilibrium in dark conditions, and becomes irrelevant at moderate forward voltage. The maximal photocurrent is determined by light absorption as a convolution of incoming spectral photon flux $\phi_{p h}(\lambda)$ at the light wavelength $\lambda$, and the external quantum efficiency (EQE), $\eta_{E Q E}$ :

$$
J_{s c}=q \int_{\lambda_{\min }}^{\lambda_{\max }} \eta_{E Q E} \phi_{p h}(\lambda) d \lambda
$$

For a semiconductor layer with absorptance $a$, the EQE relates to internal quantum efficiency (IQE) as

$$
\eta_{E Q E}=a \eta_{I Q E}
$$

$J_{r e c}(V)$ is the recombination current density that may be described with a bimolecular recombination model. This current is associated with a recombination rate defined as:

$$
U_{r e c}(V)=B n p=B p_{0} n_{0} e^{q V / m k_{B} T}
$$

The latter term includes a recombination constant, $B$, the equilibrium carrier densities $n_{0}$ and $p_{0}$, and the enhanced minority carrier concentration $n=n_{0} e^{q V / m k_{B} T}$ at voltage $V$, considering a diode ideality factor $m$. Then, the current density displays the well known diode-like behavior:

$$
J=J_{s c}+J_{0}\left(1-e^{\frac{q V}{m k_{B} T}}\right)
$$

This expression means that the solar cell behaves as a diode in the dark which facilitates current in forward bias, when Fermi levels of carriers approach the energy level of a selective contact, and conversely blocks the reverse current, as recombination is prevented and only thermal generation current exists. Under illumination, the photogenerated current is extracted until countered by an internal recombination current, which determines the open-circuit voltage. Neglecting thermal generation, $V_{o c}$ is given by:

$$
V_{o c}=\frac{m k_{B} T}{q} \ln \left(\frac{J_{s c}}{J_{0}}\right)
$$

The model of Eq. (29) was adopted by (Shockley and Queisser, 1961) (SQ) to establish the maximum power conversion efficiency of classical solar cells consisting of a single absorber of bandgap $E_{g}$ and with internal quantum efficiency of 1 . The 
maximal efficiency is limited to the theoretical value of $31 \%$. In the presence of internal resistance effects the rectifying characteristic of Eq. (29) is degraded and the $F F$ is lowered considerably. The SQ efficiency increases when several semiconductors are used to selectively absorb the solar spectrum in a tandem configuration, as discussed in section 7.2.

We have already argued that the built-in field may be confined to a narrow region close to the contact, Fig. 3, or be distributed inside the light absorber layer, Fig. 5. The main difference between these models is the way to transport charges to the respective extraction contacts and avoiding recombination. In the absence of an applied field the carrier extraction occurs entirely by diffusion. However, if the diffusion length (the average distance for photogenerated carriers to recombine under diffusion regime) is short, carrier extraction needs to be accelerated by a drift field. In the case of standard ferroelectric materials, free carrier lifetimes are quite short, e.g., 3 ns in BFO (Sheu et al., 2012), so that the presence of a strong electric field is required as explained in the next section. However, longer lifetimes in the $\mu$ s time scale have been reported in (Anshul et al., 2013). These disparate experimental results may be strongly dependent on the internal distribution of the electric field in each sample.
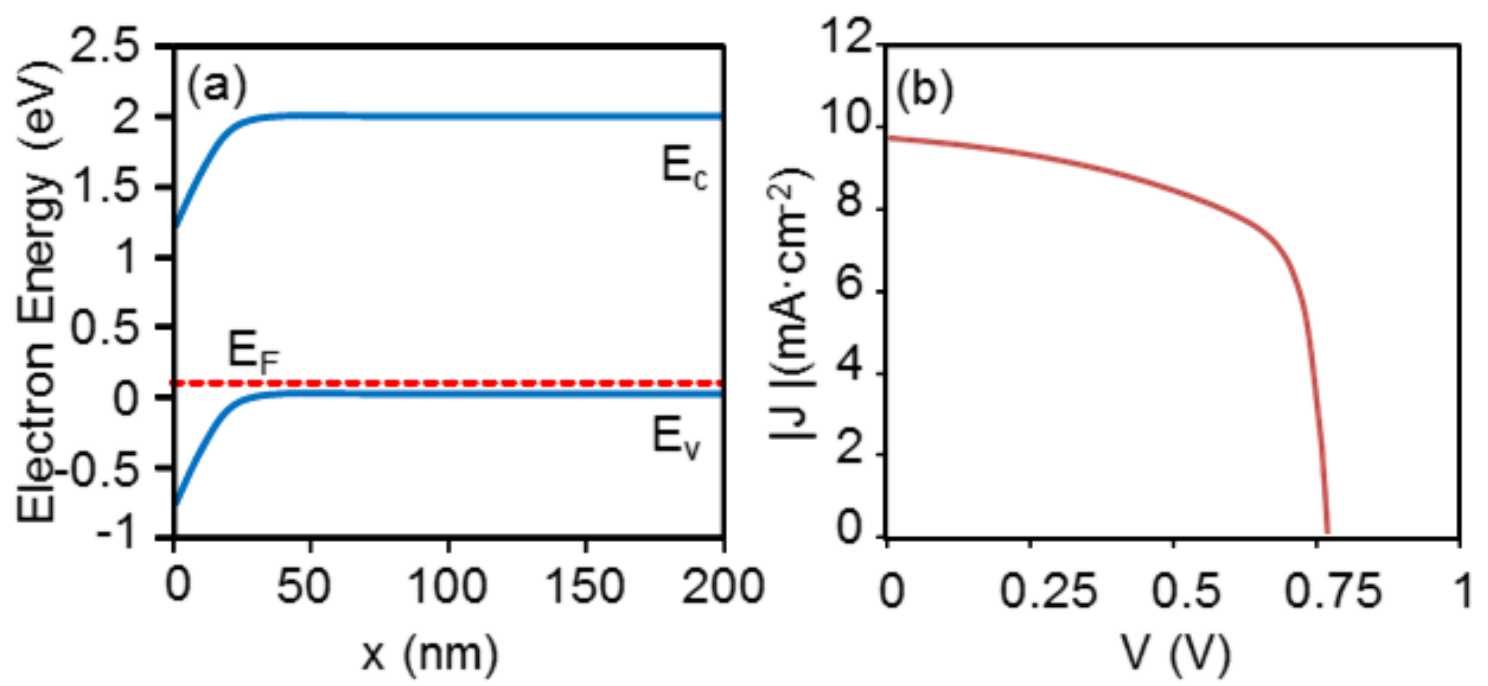

Fig. 6. (a) Band diagram for a Schottky barrier solar cell in the dark. (b) Corresponding current density-voltage characteristic. The parameters used in the numerical simulation are reported in the Table 1 of SI.

An important PV model is based on the Schottky barrier formed at the contact. In Fig. 6, a p-type semiconductor is shown with an ohmic contact for the majority carriers, at $x=d$, and a rectifying contact for the minority carriers at $x=0$. The built-in potential of the Schottky barrier in equilibrium is given by the difference in work function between the metal contact, $\Phi_{1}$, and the one of the semiconductor, $\Phi_{s c}=\Phi_{2}$, where $\Phi_{2}$ is the 
work function of the metal at $x=d$ :

$$
V_{b i}=\frac{1}{q}\left(\Phi_{1}-\Phi_{s c}\right)
$$

It is important to remark that Eq. (31) assumes vacuum level alignment after the formation of the interface, that is, all the difference of work functions is established in the semiconductor space charge layer. If a dipole of energy step $\Delta$ is formed at the metal-semiconductor interface, then the internal built-in voltage is modified as follows, (see (Bisquert, 2014) for a detailed description)

$$
V_{b i}^{\prime}=\frac{1}{q}\left(\Phi_{1}-\Delta-\Phi_{s c}\right)=V_{b i}-\frac{\Delta}{q}
$$

Concerning the operation of the Schottky barrier solar cell shown in Fig. 6, a simple model approach assumes fast charge separation in the space charge layer (SCL) (Landsberg and Klimpke, 1977), while in the neutral layer, collection is determined by the diffusion length as in Gärtner's model (Gärtner, 1959). At the rectifying junction, illumination induces a separation of the minority carrier Fermi level from its equilibrium position, which gives rise to the photovoltage. It should be remarked that Schottky barrier solar cells usually exhibit a low fill factor, see Fig. 6b, due to the fact that the increase of forward voltage reduces the extent of space charge layer, increasing recombination exponentially while the photo-generated current drops.

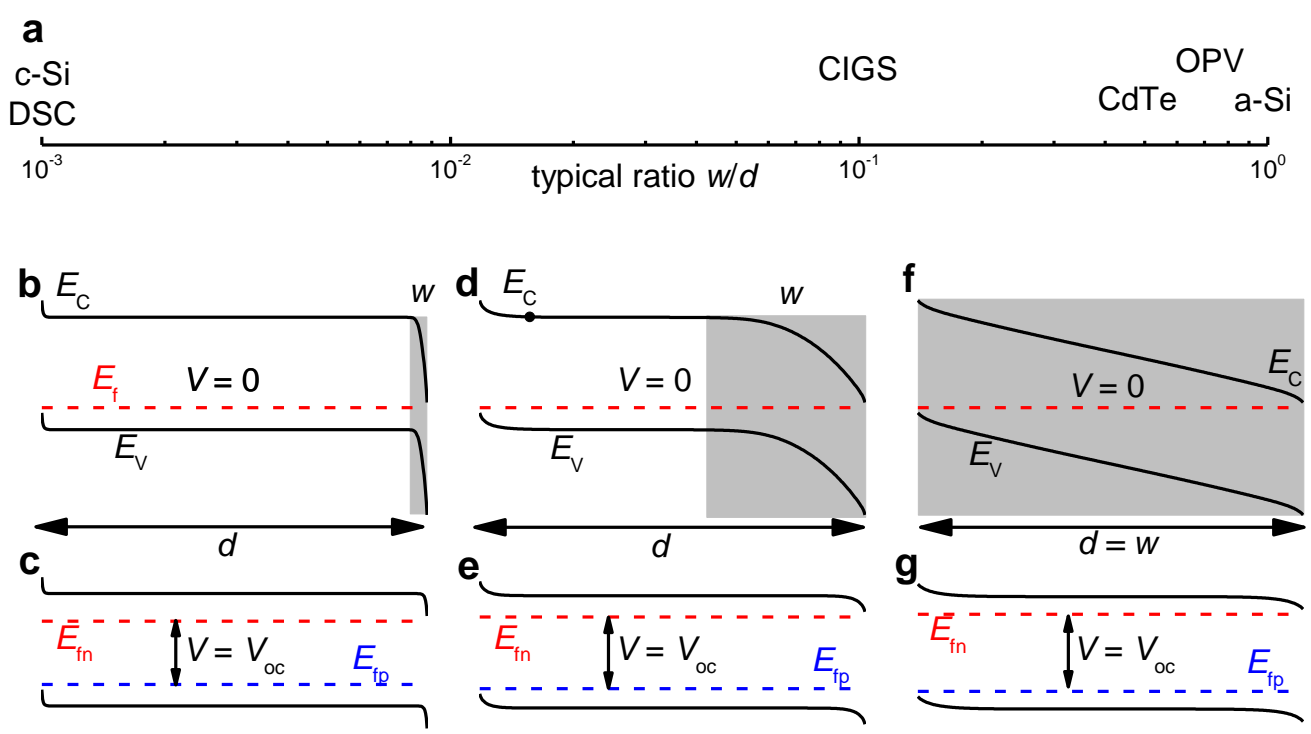

Fig. 7. a The typical ratio of space charge region with a width $w$ and thickness $d$ for different solar cell technologies, such as c-Si, DSC, CIGS and organic photovoltaics (OPV). Band diagrams of solar cells with small $w / d(\mathrm{~b}, \mathrm{c})$, intermediate $w / d(\mathrm{~d}, \mathrm{e})$ and $w=d$ (f, g) at short circuit in the dark (b, d, f) and open circuit (c, e, g). The grey areas represent the main space charge regions in the different diagrams at short circuit. 
Reprinted from (Kirchartz et al., 2015).

Different types of space charge distribution in the solar cell are summarized in Fig. 7, both in the dark, and at open-circuit condition under illumination (Kirchartz et al., 2015). A useful criterion to determine the main mechanism of charge extraction is the ratio between the width $w$ of the space charge region and the absorber thickness $d$. Crystalline $\mathrm{Si}$ (c-Si) as well as DSCs are typical examples of solar cells that have a tiny space charge region relative to the total absorber width (Fig. 7b,c). Note that c-Si solar cells are usually termed p-n junction solar cells, however the p-n junction is a rather thin selective contact and the extension of the associated drift field is insignificant. Fig. 7d,e corresponds to the Schottky barrier solar cell previously discussed. For typical inorganic thin film solar cells, the ratio $w / d$ varies from around 1/10 in $\mathrm{Cu}(\mathrm{In}, \mathrm{Ga}) \mathrm{Se}_{2}$ (CIGS) to 1 in fully depleted devices like amorphous or microcrystalline $\mathrm{Si}$ (a-Si or $\mu \mathrm{c}-$ $\mathrm{Si}$ ), Fig. 7f and g. In the case where the device is fully depleted at short circuit, the builtin electric field extends over the whole absorber, (Schiff, 2003). Fully depleted cells are sometimes denoted p-i-n type.

One of the limitations to the efficiency of the solar cell is the open-circuit voltage. As observed in Fig. 7, for all conventional solar cells the open-circuit voltage corresponds to the situation in which the Fermi levels are nearly flat. The conduction and valence band edges have a very large density of states and it takes a very large carrier density for the Fermi levels to go deep inside the bands. This is not achievable at a reasonable illumination level close to 1 sun. Thus the photovoltage is limited to $q V_{o c}<E_{g}$. This is the maximal allowed splitting of the Fermi levels.

Importantly, we also need to examine the maximum power point of an illuminated solar cell. Extraction barriers $\Phi_{B, n}$ and $\Phi_{B, p}$ need to have the signs indicated in Fig. 5, otherwise charge extraction will be blocked at the contacts. The photovoltage will be limited to the built-in voltage in cases where the electrodes accept both electrons and holes equally well. The photovoltage can in principle exceed the built-in voltage if recombination of minorities is suppressed at the electrodes. In technologically relevant solar cells $V_{o c}<V_{b i}$ is typically given although in many cases care is taken to suppress recombination at the electrodes. The built-in voltage $V_{b i}$ ensures that the applied forward bias $V$ can drop somewhere. That is, the VL can be modified as an effect of light without creating kinetic barriers, as long as $V_{b i}-V$ is still positive (Rau et al., 2003, Turrión et al., 2003,Kirchartz and Rau, 2011). Thus, the built-in voltage serves an important role even if like in c-Si solar cells, charge collection is almost entirely driven by diffusion. 


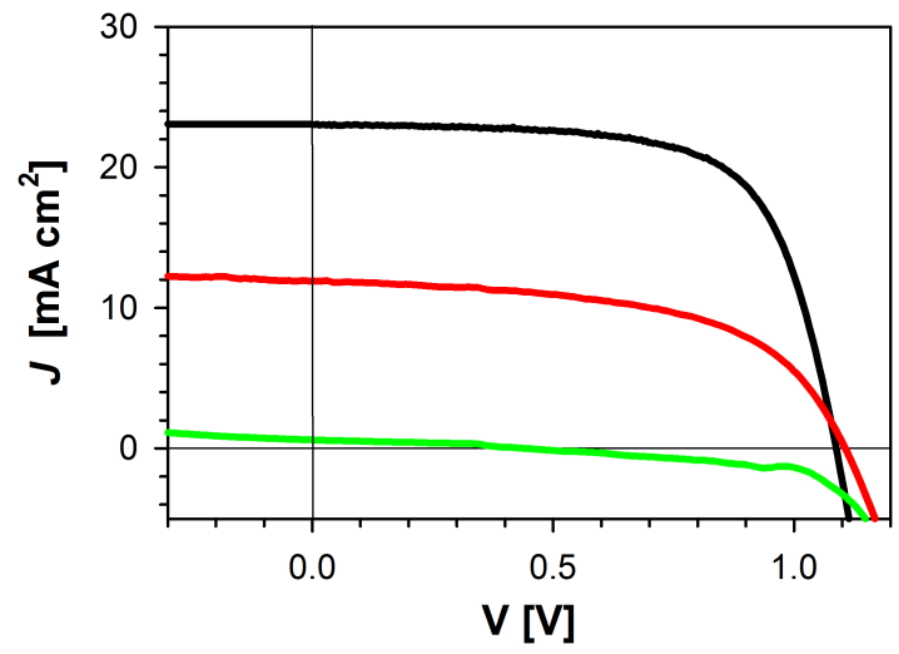

Fig. 8. Current density/voltage characteristics of $\mathrm{MAPbI}_{3}$ perovskite solar cells with different electron contact, in decreasing order of photocurrent: $\mathrm{SnO}_{2}, \mathrm{TiO}_{2}, \mathrm{Nb}_{2} \mathrm{O}_{5}$, and spiro-OMeTAD hole contact, at one AM1.5 illumination. Reprinted with permission from (Guerrero et al., 2016).

Table 1: Summary of device efficiency of devices shown in Fig. 8.

\begin{tabular}{cccccc}
\hline Device & $\begin{array}{c}\text { Electron } \\
\text { contact }\end{array}$ & $\begin{array}{c}\boldsymbol{J}_{\text {sc }} \\
{\left[\mathbf{m A} / \mathbf{c m}^{-2}\right]}\end{array}$ & $\begin{array}{c}\boldsymbol{V}_{\text {oc }} \\
{[\mathbf{V}]}\end{array}$ & FF & $\begin{array}{c}\text { PCE } \\
{[\%]}\end{array}$ \\
\hline 1 & $\mathrm{SnO}_{2}$ & 23.06 & 1.09 & 0.68 & 16.92 \\
2 & $\mathrm{TiO}_{2}$ & 11.60 & 1.08 & 0.78 & 9.83 \\
3 & $\mathrm{Nb}_{2} \mathrm{O}_{5}$ & 0.60 & 1.07 & 0.30 & 0.20 \\
\hline
\end{tabular}

A representative example of high performance solar cell is shown in Fig. 8. The $J-V$ characteristics is displayed for different $\mathrm{MAPbI}_{3}$ solar cells in which the central perovskite layer and hole conductor contact are formed by a similar procedure, but the electron contacts are prepared by an atomic layer deposition (ALD) method of different metal oxide materials. Performance parameters are indicated in Table 1. The highest efficiency is obtained in the sample with the $\mathrm{SnO}_{2}$ contact. As in Fig. 8, the top performing solar cell shows excellent rectification that is controlled by recombination as indicated in Eq. (24). In this case, the photocurrent is nearly constant in the region of zero and reverse bias, since the excellent properties of selective contacts and long carrier diffusion length make it possible to extract all internally generated photocurrent. However, for a worse $\mathrm{TiO}_{2}$ contact, the photocurrent is severely decreased. In the case of a seriously degraded $\mathrm{Nb}_{2} \mathrm{O}_{5}$ contact, the photocurrent is relatively small due to deficient extraction of carriers. In addition, the rectification characteristic is not maintained in the first quadrant, and consequently the fill factor decreases to a very low 
value of 0.30 .

\section{Phenomenology of ferroelectric solar cells}

In this section, we present a general overview of typical PV behavior observed in solar cells made with a ferroelectric semiconductor as the central absorber material. We describe the actual phenomenology and results that have been generally obtained so far, while specific physical mechanisms and characteristics will be reviewed in the next section.

Fig. 9a shows the photocurrent in epitaxial BFO thin films of $170 \mathrm{~nm}$ on STO/SRO as the bottom electrode and ITO top electrode. The current is reported in the as-prepared sample and after poling it in opposite directions. In general, we define up, upward or positive poling when the remnant polarization points to the top electrode and down, downward or negative poling when the remnant polarization points to the bottom electrode (see Fig. 10). The current density in all cases shows an ohmic characteristic, i.e. it is linear with the voltage and can be described with an expression of the type

$$
J=-J_{p h}+\sigma F
$$

where $F$ is the internal electric field, $J_{p h}$ is the photocurrent, and $\sigma$ is the combined electron and hole conductivity, that is composed of the dark conductivity, $\sigma_{d}$, and the photoconductivity, $\sigma_{p h}$.

$$
\sigma=\sigma_{d}+\sigma_{p h}
$$

In terms of the applied voltage $V$ and the effective built-in voltage of the device, we have

$$
J=-J_{p h}+\frac{\sigma}{d}\left(V-V_{b i}\right)
$$



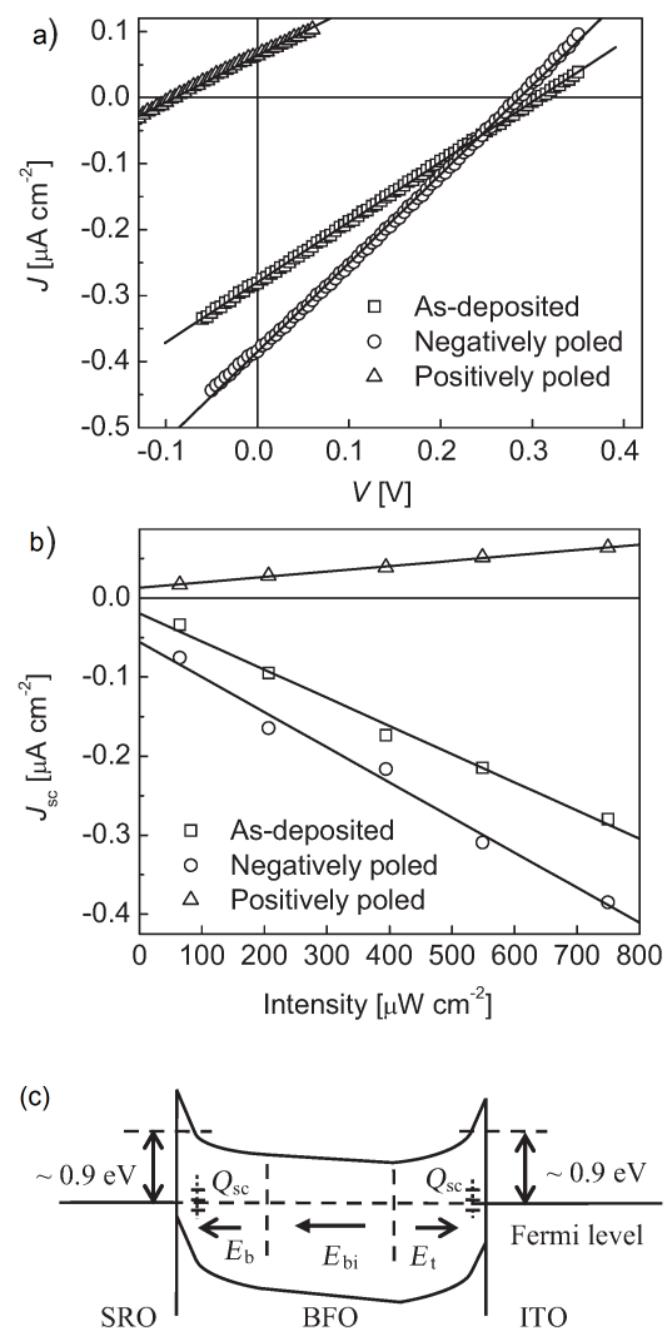

Fig. 9. Photovoltaic response for ITO/BFO (170 nm)/SRO/STO(001). a) J-V characteristics measured with incident light of $435 \mathrm{~nm}$ at $750 \mathrm{~mW} \mathrm{~cm}{ }^{-2}$ for the films without poling and after positive and negative poling. b) Short-circuit current density, $J_{s c}$ as a function of light intensity. c) Schematic of energy-band alignment for the ITO/BFO/SRO capacitor according to the fixed separation model of the metal induced gap states (MIGS). $E_{\mathrm{b}}$ and $E_{\mathrm{t}}$ are the built-in field at the bottom- and top-electrode interfaces, respectively. $E_{\mathrm{bi}}$ is the unswitchable built-in field in the film bulk possibly due to non-uniform distribution of defects. $Q_{s c}$ denotes the gap-state charge density. Reprinted from (Ji et al., 2010).

Thus, the open-circuit voltage is

$$
V_{o c}=\frac{J_{p h} d}{\sigma}+V_{b i}
$$

Fig. $9 \mathrm{~b}$ suggests that the short-circuit current depends linearly on photogeneration rate $G$ as

$$
J_{s c}=-k G-J_{d}
$$


$J_{p h}$ and $J_{d}$ are given by

$$
\begin{aligned}
& J_{p h}=-\frac{\sigma_{p h}}{d} V_{b i}+k G \\
& J_{d}=\frac{\sigma_{d}}{d} V_{b i}
\end{aligned}
$$

Therefore

$$
J=-k G+\frac{\sigma V}{d}-J_{d}
$$

The behavior observed in Fig. 9, consisting of a linear $J-V$ plot, is rather characteristic in many ferroelectric materials such as $\mathrm{BFO}$ and $\mathrm{BaTiO}_{3}$ (Zenkevich et al., 2014). The photocurrent can be switched with the polarization sign, so that the parameter $k$ in Eq. (37) depends on the poling history of the sample. Fig. 10 shows that a PZT sample of thickness $300 \mathrm{~nm}$ (Yang et al., 2000) delivers a photocurrent of 0.15 $\mu \mathrm{Acm}^{-2}$, and a photovoltage that is in the range of $1.2 \mathrm{~V}$. The estimated conductivity and photoconductivity for these PZT films, according to Eq. (35) neglecting effective built-in voltage, were $4.8 \times 10^{-12}$ and $3.2 \times 10^{-11} \Omega^{-1} \mathrm{~cm}^{-1}$, respectively.

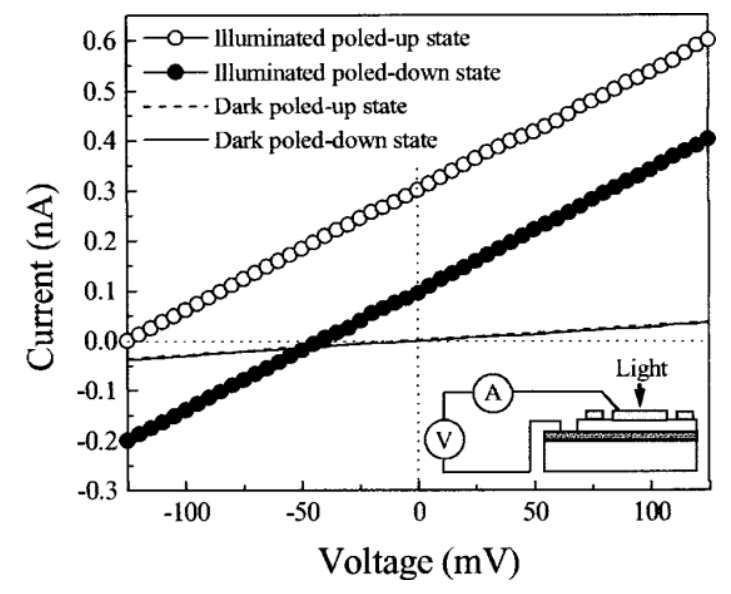




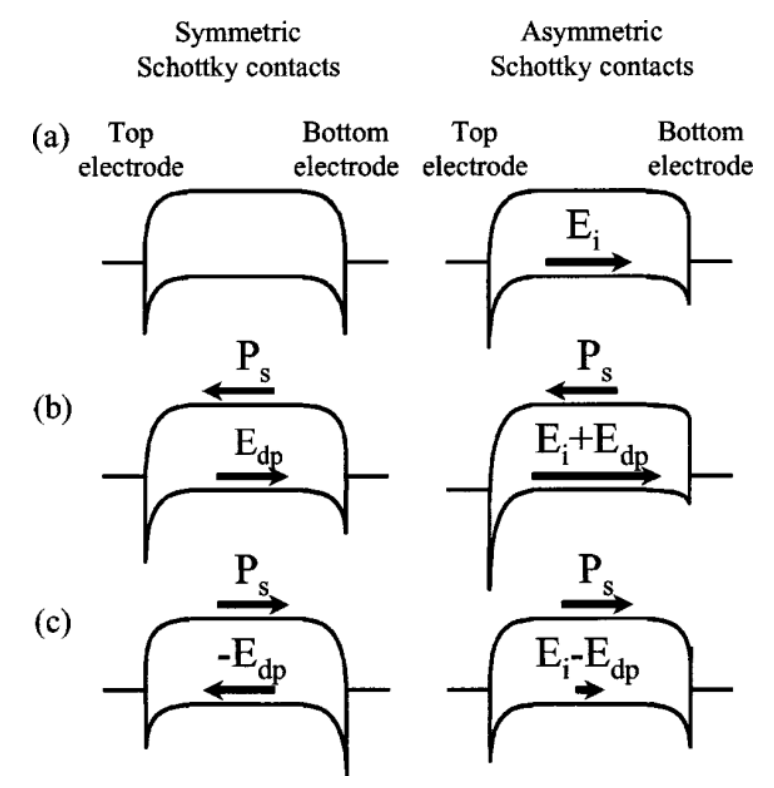

Fig. 10. Top: Dark currents and photocurrents of $\mathrm{PbZr}_{0.53} \mathrm{Ti}_{0.47} \mathrm{O}_{3}$ (PZT) 300-nm-thick film, on $\mathrm{Pt} / \mathrm{Ti} / \mathrm{SiO}_{2} / \mathrm{Si}$ substrates and top $10 \mathrm{~nm}$ thickness Pt electrode. Bottom: (a) Model of Schottky barriers modified by (b) positive or up and (c) negative or down ferroelectric polarization. Reproduced from (Yang et al., 2000).
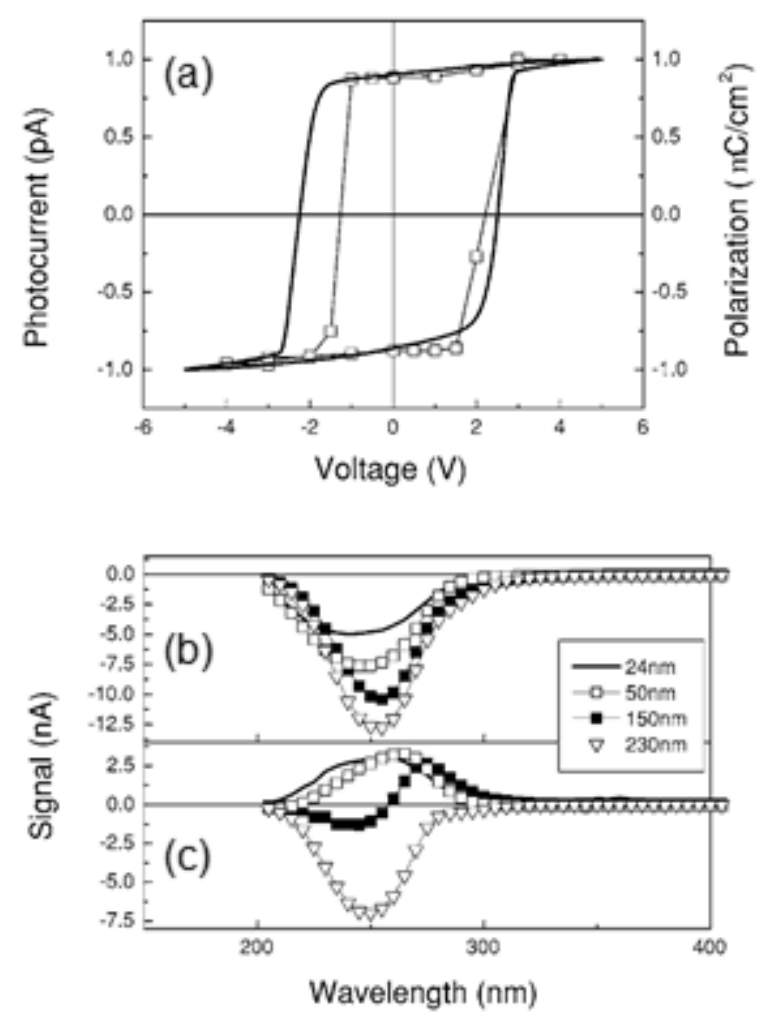

Fig. 11. The behavior of the photocurrent signal in PZT films sandwiched between SRO top electrode and a semitransparent Pt layer. (a) when the ferroelectric polarization 
describes a full hysteresis loop. Both ferroelectric polarization (measured at $1 \mathrm{kHz}$ ) and photocurrent signal were normalized to their respective maximum value. (b), (c) Thickness dependence of the photocurrent signal for the two orientations of the polarization: up and down, respectively. The polarization is considered "up" when it is oriented toward the top SRO electrode. Reprinted from (Pintilie et al., 2007).

However, the origin of the photocurrent switching is not generally understood and it is even difficult to determine whether the effect is due to a bulk-conduction or an interface-barrier controlled mechanism, as discussed in the following. In Fig. 11a, the photocurrent in a SRO/PZT/Pt sample is represented as a function of the poling voltage together with the dynamic hysteresis loop (Pintilie et al., 2007). It is observed that the photocurrent describes exactly the same hysteresis loop as the polarization. The photocurrent loop is almost rectangular with sharp switching and flat saturation polarization for both positive and negative branches. These observations indicate that the photocurrent is directly related to the ferroelectric polarization. According to Eq. (38), if the conductivity under illumination is dominated by the dark conductivity ( $J_{p h} \approx k G$ ), the photocurrent should increase with film thickness due to a larger light absorption and generation rate $G$. However, a study of the photocurrent dependence on film thickness and polarization, Fig. 11b, shows that the sample does not always show consistent trends. For one orientation of the polarization, the photocurrent is almost proportional to the thickness of the PZT films, in agreement with a possible bulk effect for the photovoltaic response, in which the increase of light absorption for thicker samples is translated into larger photocurrent. On the other hand, in the case of the opposite polarization orientation, the apparent thickness dependence disappears, and the photocurrent shows a random behavior with respect to the thickness in this case. It was suggested that the photocurrent is not simply controlled by an electric field that depends on polarization orientation. A non-uniform distribution of charged traps might produce an internal field that controls these phenomena, affecting the polarization switching by pinning domains or by inducing back switching after removal of the poling field (Pintilie et al., 2007). 

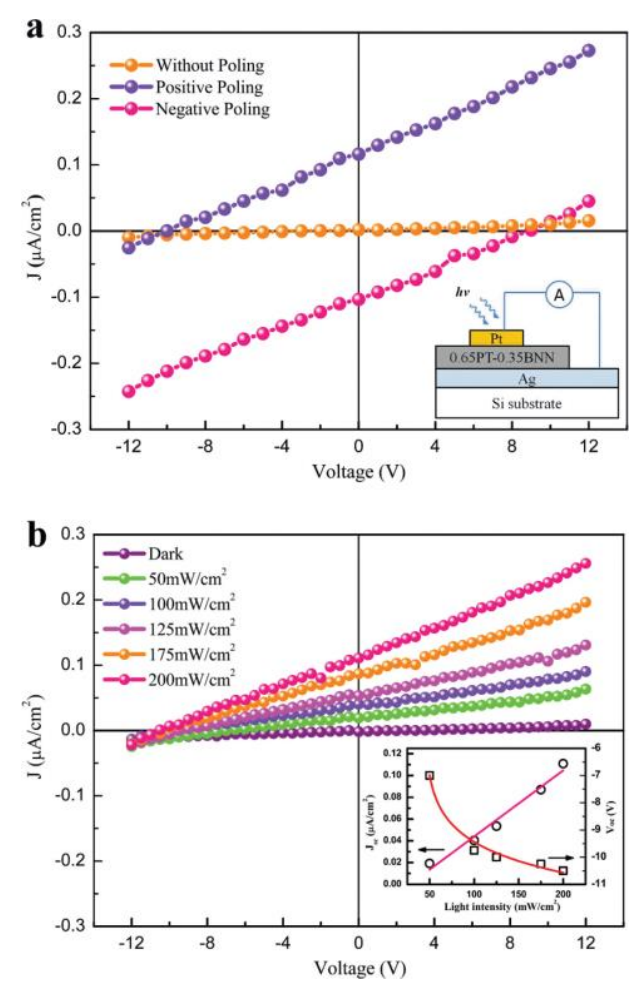

Fig. 12. a) $J-V$ characteristics of $50-\mu \mathrm{m}$-thick $\mathrm{Pt} / 0.65 \mathrm{PT}-0.35 \mathrm{BNN} / \mathrm{Ag}$ sample $\left(\mathrm{PbTiO}_{3}\right.$ $\left.-\mathrm{Bi}\left(\mathrm{Ni}_{2 / 3+\mathrm{x}} \mathrm{Nb}_{1 / 3-\mathrm{x}}\right) \mathrm{O}_{3-\delta}\right)$ measured with white light of $200 \mathrm{~mW} \mathrm{~cm}^{-2}$. The inset displays the $\mathrm{Pt} / 0.65 \mathrm{PT}-0.35 \mathrm{BNN} / \mathrm{Ag}$ structure of the photovoltaic devices. b) $J-V$ curves of $\mathrm{Pt} / 0.65 \mathrm{PT}-0.35 \mathrm{BNN} / \mathrm{Ag}$ device under various light intensities after positive poling. The inset shows the short-circuit photocurrent density $\left(J_{s c}\right)$ and the open-circuit voltage $\left(V_{o c}\right)$ as a function of light intensity. Reprinted from (Liu et al., 2015).
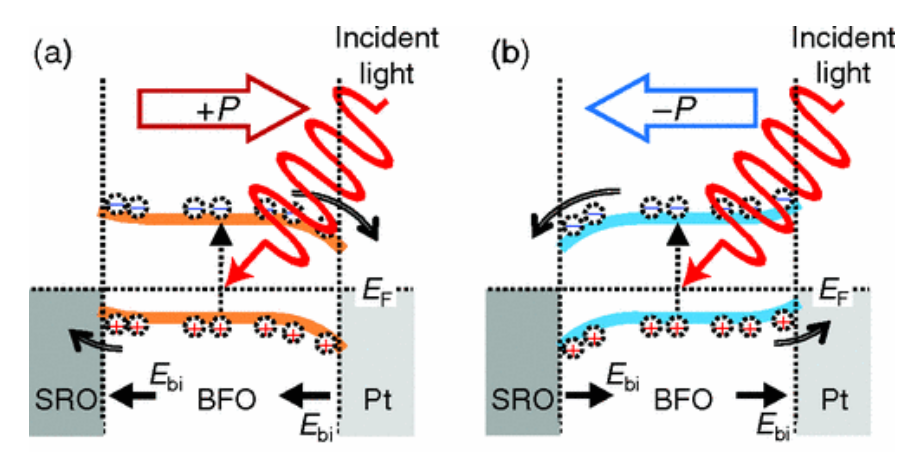

is: Hole, : Electron

Fig. 13. Schematic description of the photovoltaic effect in a ferroelectric device for (a) the upward and (b) downward polarization states. The slope of the band edges at the interfaces can generate a built-in field $\left(E_{b i}\right)$. Reprinted from (Lee et al., 2011). 


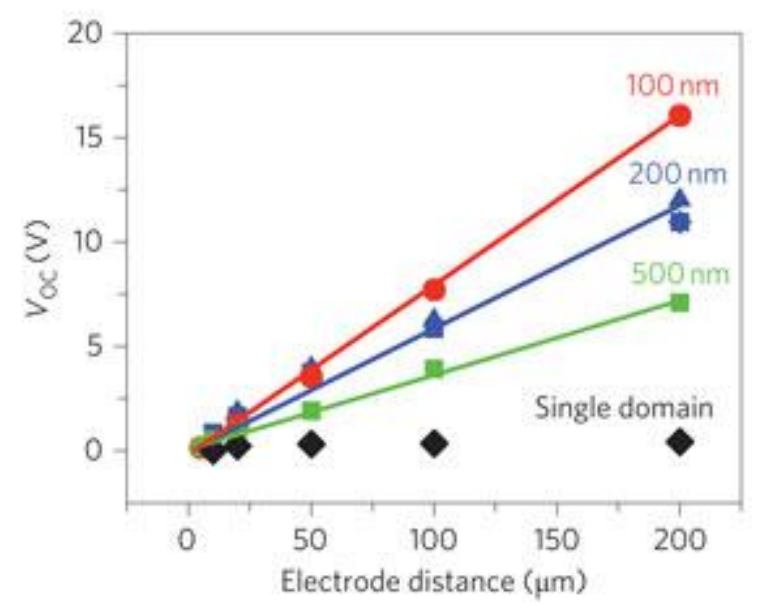

Fig. 14. Study of the evolution of $V_{o c}$ in symmetric $\mathrm{Pt} / \mathrm{BFO} / \mathrm{Pt}$ capacitors as a function of electrode spacing for four different samples: $71^{\circ}$ domain-walls samples with thicknesses of $100 \mathrm{~nm}$ (red), $200 \mathrm{~nm}$ (blue) and $500 \mathrm{~nm}$ (green) as well as a monodomain BFO film having no domain walls (black). Reproduced from (Yang et al., 2010).

Fig. 12 illustrates additionally the typical behavior observed in ferroelectric layers. First of all, we remark the total inversion of the $J-V$ curve by poling, as noted before. This effect indicates that the operation of these cells is very different from the picture established in Fig. 4 and 7, in which each contact has a fixed extraction property for either electrons or holes. Although the contacts in Fig. 12 are asymmetric (Pt and Ag), the charge extraction properties of the contacts of these devices are not permanent but can be modified by the applied polarization, as shown in Fig. 12a providing a nearly symmetric photovoltages. This is usually explained in terms of a model in which the contact Schottky barriers are modified by poling, as shown in Fig. 9c, Fig. 10b and Fig. 13 and discussed in section 4.2, see Eq. 44. The photocurrent in Fig. 12 is proportional to light intensity, as observed and explained in many publications (Grekov et al., 1970,Glass et al., 1974,Gunter, 1978,Sturman and Fridkin, 1992,Guo et al., 2013,Wu et al., 2014). Another noteworthy feature in Fig. 12 is the value of a photovoltage of $10 \mathrm{~V}$ at visible wavelengths $(400-780 \mathrm{~nm})$ with a light intensity of $200 \mathrm{~mW} \mathrm{~cm}{ }^{-2}$. The observation of very large photovoltages in ferroelectric materials has been known for many decades (Starkiewicz et al., 1946,Goldstein and Pensak, 1959,Grekov et al., 1970,Brody, 1973,Glass et al., 1974). Recent examples of large photovoltages are the measurements of $7 \mathrm{~V}$ in $25 \mu \mathrm{m}(\mathrm{Pb}, \mathrm{La})(\mathrm{Zr}, \mathrm{Ti}) \mathrm{O}_{3}$ at $1.5 \mathrm{~mW} \mathrm{~cm}^{-2}$ (Pintilie, 2011), 8.8 $\mathrm{V}$ with $4 \mathrm{~mW} \mathrm{~cm}{ }^{-2} \mathrm{UV}$ light on $\mathrm{KBiFe}_{2} \mathrm{O}_{5}$ (Zhang et al., 2013,Zhai et al., 2015) and 3.6 $\mathrm{V}$ in $50 \mu \mathrm{m} \mathrm{BiFeO}$, see Fig. 14 (Yang et al., 2010).

We have previously commented on the linear $J-V$ characteristics of many reported ferroelectric solar cell materials, which is in contrast to the exponential diode characteristic that is typical of high performance solar cells. However, in recent years materials demonstrating rectifying behavior have been reported, as shown in Fig. 15. In 
addition, the diode characteristic is completely switchable by the applied electric field (Yi et al., 2011). (Yang et al., 2016) showed that the $J-V$ characteristic of $\mathrm{La}_{2 / 3} \mathrm{Sr}_{1 / 3} \mathrm{MnO}_{3} / \mathrm{BFO} / \mathrm{ITO}$ at one sun illumination could be switched by polarization. In the upward direction, the curve is rectifying and the photocurrent is proportional to light intensity, while in the downward state the curve is linear. The photocurrent is always opposite to the polarization direction. These results are explained by a modification of Schottky barriers induced by polarization.

A general improvement of diode characteristics has been obtained in recent years in accordance with reported significant photovoltaic efficiencies of 1\% PCE, and sizable photocurrents. (Yang et al., 2009) have reported photovoltaic characteristics of ITO/BFO (>200 nm) / SRO samples, Fig. 16a. $J-V$ curves taken both in the dark and under 2.85 suns show good diode rectifying behavior, with high photovoltage of 0.8-0.9 $\mathrm{V}$ and short-circuit current densities of $1.5 \mathrm{~mA} \mathrm{~cm}^{-2}$. In these results BFO shows a direct bandgap of $2.67 \mathrm{eV}$. The direction of short-circuit current flow went in all cases from the ITO, through the BFO, to the SRO. These results were explained in terms of a Schottky barrier formed at the ITO-BFO interface that extends over several hundred nanometers. On the other hand, (Zhou et al., 2014) showed that the photovoltage in BFO is independent of the crystal orientation, consistent with a control by the interfacial barriers. (Hu et al., 2016) reported solar cells of relatively high $1.25 \%$ efficiency among the ferroelectric solar cells. The $J-V$ characteristic indicates diode like behavior but internal resistance seems to be quite large, Fig. 16b.

Finally, Fig. 17 shows the properties and performance of a multi-absorber system, based on BFCO (as mentioned in the Introduction) that forms current multiferroic champion devices (Nechache et al., 2015). Device M1 yielded $J_{s c}=20.6 \mathrm{~mA} \mathrm{~cm}{ }^{-2}$, $V_{o c}=0.84 \mathrm{~V}$, and FF $=0.46$, and PCE of $8.1 \%$. For device M2 $J_{s c}=23.6 \mathrm{~mA} \mathrm{~cm}{ }^{-2}$, $V_{o c}=0.56 \mathrm{~V}$ and $\mathrm{FF}=0.33$, and $\mathrm{PCE}=4.3 \%$ were obtained. 

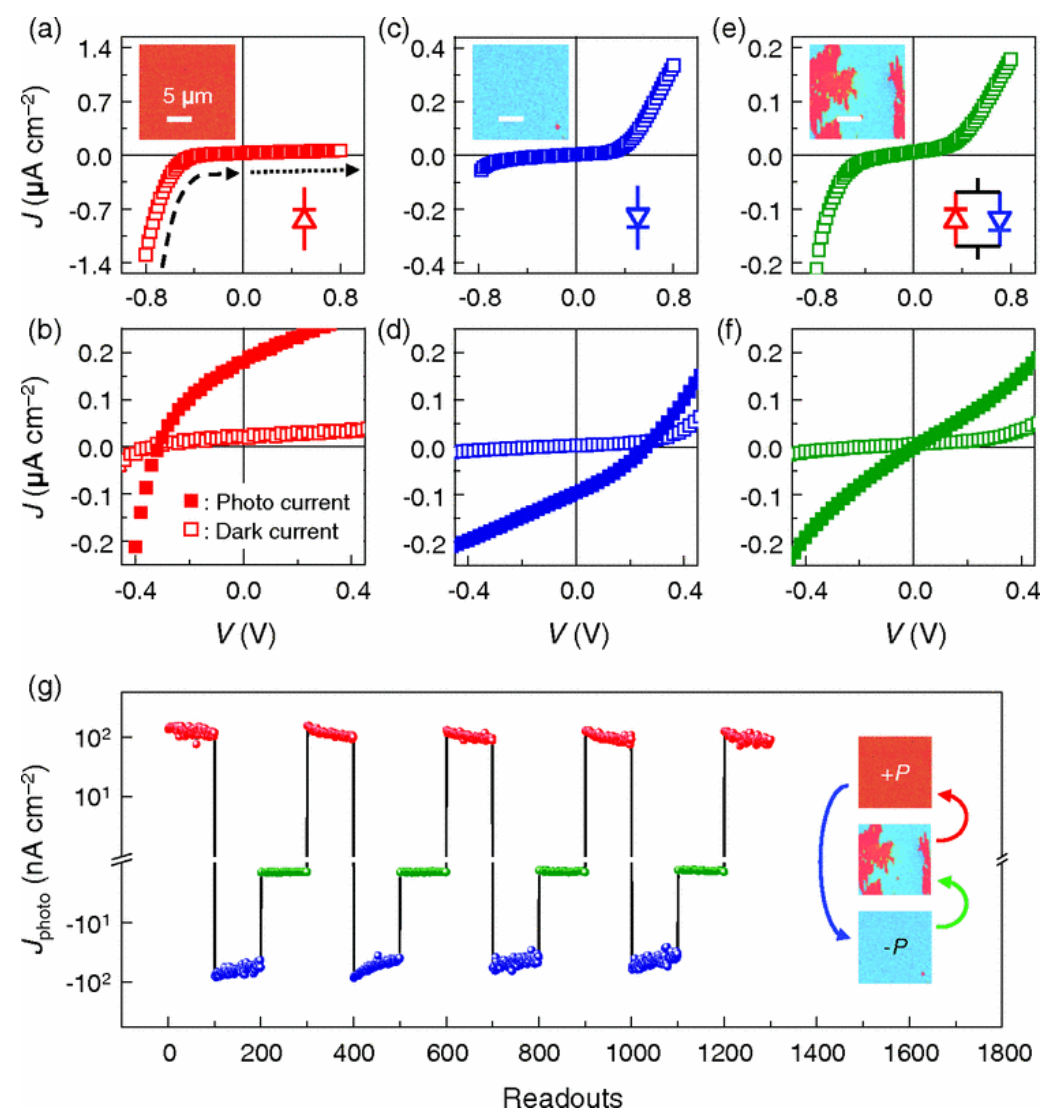

Fig. 15. $J-V$ curves (open squares) for a $\mathrm{Pt} / \mathrm{BiFeO}_{3} / \mathrm{SrRuO}_{3}$ device with a $\mathrm{BiFeO}_{3}$ thickness of $400 \mathrm{~nm}$, measured in the dark for the (a) upward-, (c) downward-, and (e) upward/downward mixed-polarization domains (Pt is the top electrode). The upper-left insets show the ferroelectric domains measured by the out-of plane piezoelectric atomic force microscope. $J-V$ curves (closed squares) for the electrically trained $\mathrm{BiFeO}_{3}$ thin capacitor measured under illumination for the (b) upward-, (d) downward-, and (f) upward/downward mixed-polarization domains. (g) Cycling test of the three. Reprinted from (Lee et al., 2011).

(a)

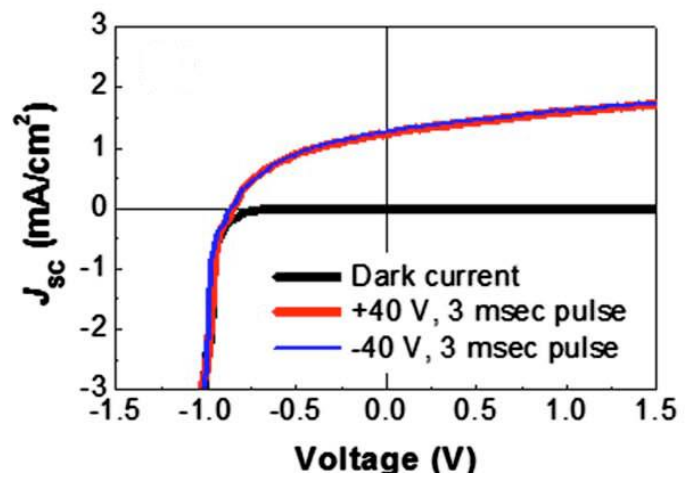

(b) 


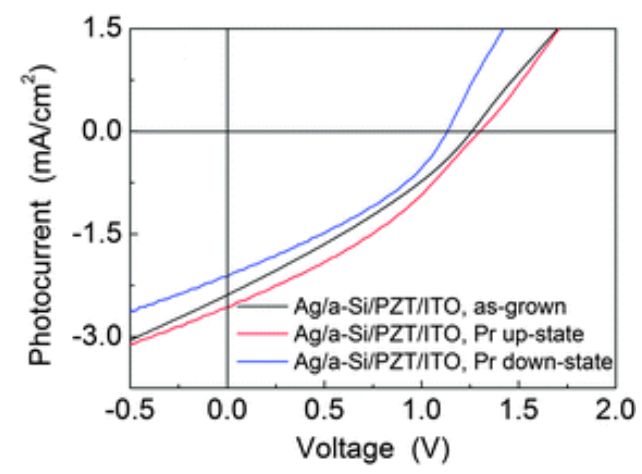

Fig. 16 (a) $J-V$ measurements at 2.85 suns of ITO/BFO( $>200 \mathrm{~nm}$ )/SRO (Yang et al., 2009) (b) $J-V$ characteristics of Ag/a-Si/PZT/ITO/glass samples illuminated under simulated standard sunlight (AM 1.5, $100 \mathrm{~mW} \mathrm{~cm}^{-2}$ ) when the PZT films were in the asgrown state (no poling voltage), remnant polarization pointing to the top electrode (upstate, $+10 \mathrm{~V}$ poling for $5 \mathrm{~s}$ ), and remnant polarization pointing to the bottom ITO electrode (down-state, $-10 \mathrm{~V}$ poling for $5 \mathrm{~s}$ ), respectively. Here, the reference voltage was defined as a positive voltage applied on the bottom ITO electrode. Reproduced from (Hu et al., 2016).
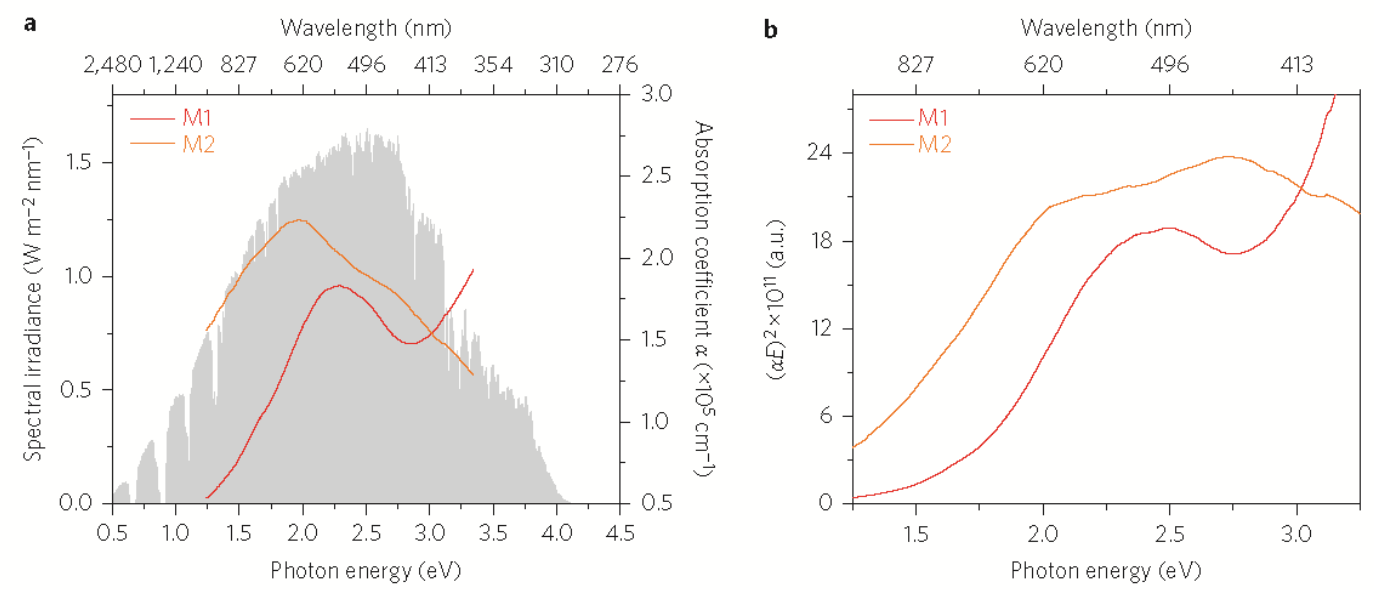

c
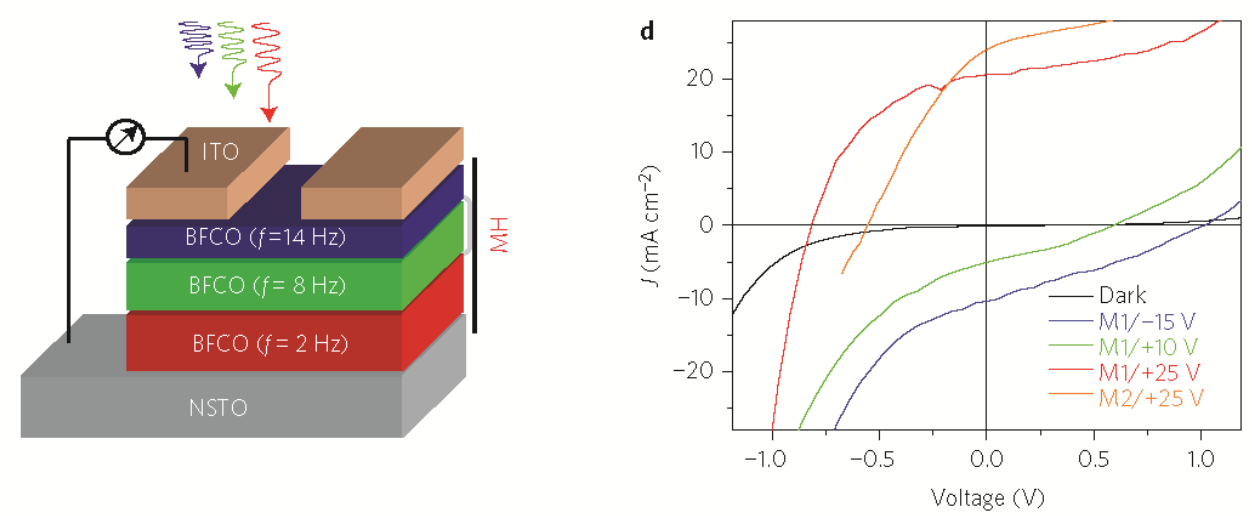

Fig. 17. (a) UV-visible absorption profiles of BFCO multilayers (M1 and M2), which indicate that the material absorbs the solar spectrum from 1.4 to $3.2 \mathrm{eV}$ (from 885 
to $388 \mathrm{~nm}$ ) in a complementary manner. (b) $(\alpha F)^{2}$ versus energy plots, in arbitrary units (a.u.). (c) Device geometry of the tested BFCO multilayer structure. (d) J-V characteristics of BFCO multilayer devices under AM1.5 G illumination. Reprinted from (Nechache et al., 2015).

\section{Ferroelectric mechanisms in solar cell devices}

The photovoltaic performance of different materials and their combinations is controlled by a wide variety of properties that must work in a beneficial way. Polarity and ferroelectricity affect fundamental aspects of the operation mechanism, as we have highlighted with many examples in the previous section. They modify the overall energy diagram by affecting the band bending and the surface barriers.

Fig. 5 shows the energy diagram of an insulator capacitor with asymmetric contacts, which is also a primitive model of a solar cell. At equilibrium, surface charges are present which are related to the bound polarization charges at the metal-ferroelectric contact as $\sigma_{p o l}=P$. Such density of charges can be determined by Eq. (7). This charge is partially compensated by free charges accumulated at each contact with surface density $\pm \sigma_{f, e q}$, as depicted in Figure 18 .

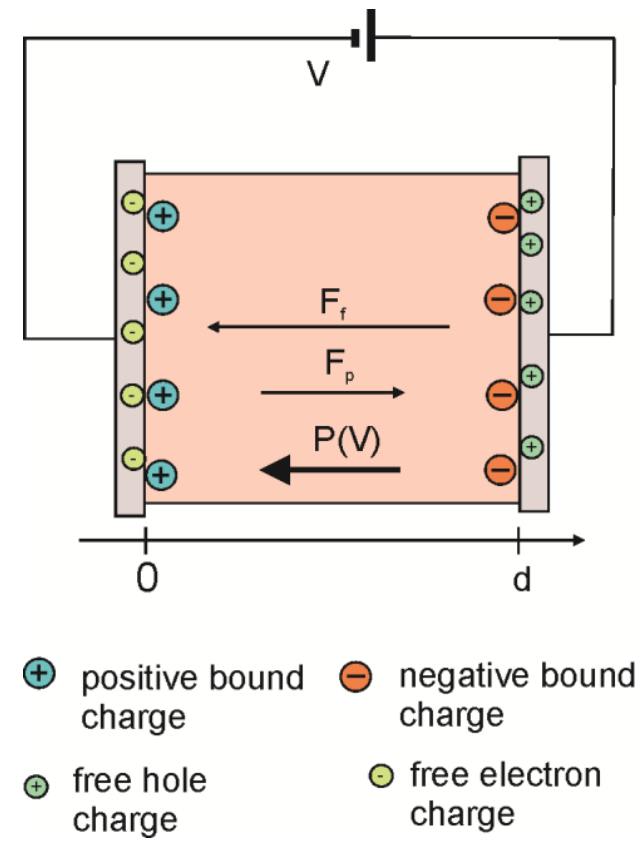

Fig. 18. Polarization of a dielectric or ferroelectric, indicating the polarization vector $P$, the electric field due to the bound charge $F_{p}$, and the electric field due to the free charge $F_{f}$.

In a solar cell device with selective contacts, we define as positive or forward bias as a negative voltage applied to the electron extraction contact (right contact in Fig. 5), or a positive voltage applied to the hole extraction contact (left contact in Fig. 5). If electrodes are symmetric, the positive voltage needs to be defined with respect to some geometry consideration, such as top or bottom electrode, as mentioned earlier. The 
effect of the positive voltage is shown at the right of Fig. 5b: it tends to flatten the conduction band, which counteracts the effect of $V_{b i}$.

\subsection{Change of injection barriers and depolarization field}

The specific mechanism of charge compensation at the external contacts has rather significant consequences for the distribution of electrical field and heights of the injection barriers in a ferroelectric solar cell. For this reason, we consider this point in some detail. When a ferroelectric material has been poled by an applied electric field, the spontaneous polarization, $P$, needs to be considered in the charge balance, as shown in Fig. 18. This polarization is assumed to be independent of the changes of voltage as long as the coercive field is not exceeded. Under a larger voltage, the polarization direction can be reversed. The positive poling is achieved by applying a reverse bias voltage in excess of the coercive voltage, while negative poling requires a forward voltage. In ferroelectric materials, the asymmetry of the polarization curve is also due to the imprint, that is a preference for one polarized state in the material (Alexe et al., 2001).

For simplicity we consider a Metal-Ferroelectric-Metal (MFM) junction with symmetric metal contacts having the same work function, see Fig. 19. As the central layer is polarized, the vacuum level (VL) is tilted even when the materials are separated, as shown in Fig. 19a. Obviously, if the system reaches equilibrium with flat Fermi level, the VL will be flattened and no electrical field exists within the device. This is because the free charges coming to the electrodes exactly cancel the polarization field $F_{p}$. However, one should take into account the important remark of (Pintilie and Alexe, 2005). The polarization bound charge is not located in the metal contact but a distance $\delta$ away from it, which may be of atomic dimension. This is due to the fact that ferroelectricity is suppressed gradually and not abruptly at the interface (Misirlioglu and Yildiz, 2014). Therefore, surface dipole layers are formed, as indicated in Fig. 19b that modify the effective surface injection barriers $\Phi_{B}$. If originally there is a built-in voltage due to different metal work functions, $V_{b i}$, it is modified by polarization at each boundary, as commented previously in Eq. (32). The modified built-in potential, $V_{b i}^{\prime}$, read:

$$
V_{b i}^{\prime}=V_{b i} \mp \frac{P_{s} \delta}{\varepsilon_{0} \varepsilon_{r}}
$$

The modification of the conduction band at $\mathrm{BiFeO}_{3} /$ metal interface can be estimated as $P_{s} \delta / \varepsilon_{0} \varepsilon_{r}= \pm 0.6 \mathrm{~V}$ for $P_{s}= \pm 65 \mu \mathrm{C} \mathrm{cm}^{-2}, \delta=1 \mathrm{~nm}$, and $\varepsilon_{r}=100$ (Lee et al., 2011). Fig. 20 shows how the change of injection barriers due to the inversion of polarization direction well explains the switch of rectification in $J-V$ curves. Using an important PV model (Liu et al., 2013,Fang et al., 2014), we will note that this is the way in which the surface Schottky barriers are modified by poling. The change of the conduction band at the contact measured by an AFM tip is illustrated in Fig. 21. 

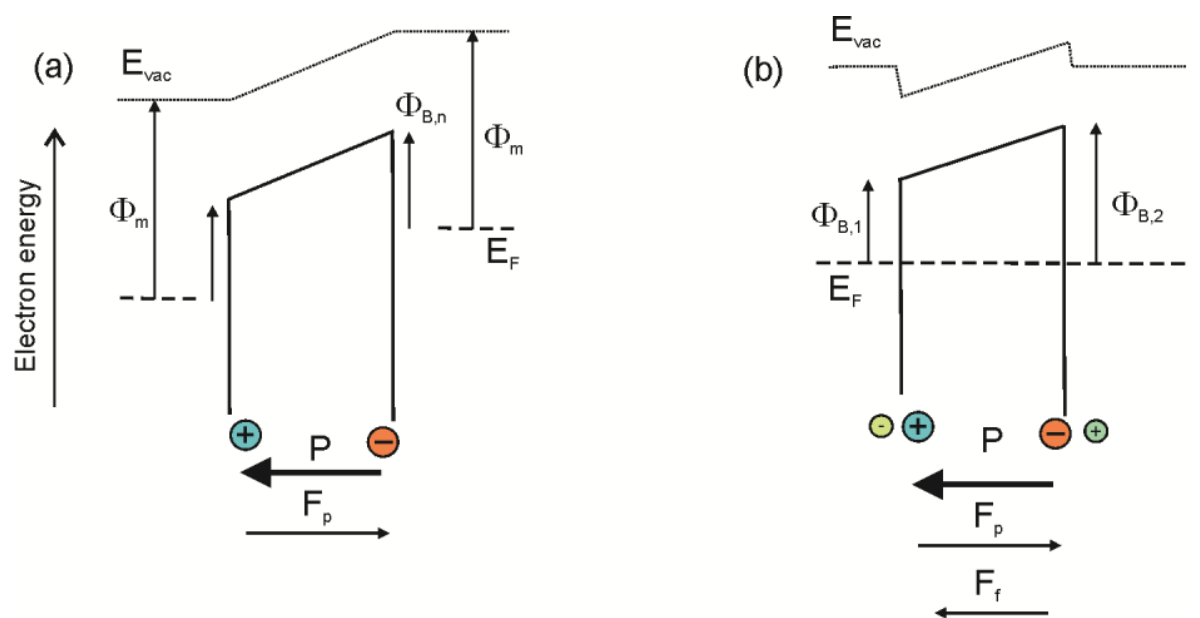

Fig. 19. (a) Energy diagram of a MFM structure with ferroelectric polarization in the central layer, indicating bound polarization charges at the edges of the layer. Two metals of same work function $\Phi_{m}$ are shown, and also the preliminary height of the electron injection barrier $\Phi_{B, n}$ is represented. (b) In equilibrium, free charges appear in the metals achieving the alignment of the Fermi levels. Dipole layers at the contacts, created by the spatial separation between the bound and the electrode charges, produce modifications of the initial injection barriers. Note that $\mathrm{V}_{\mathrm{bi}}=0$ is conserved along the VL.

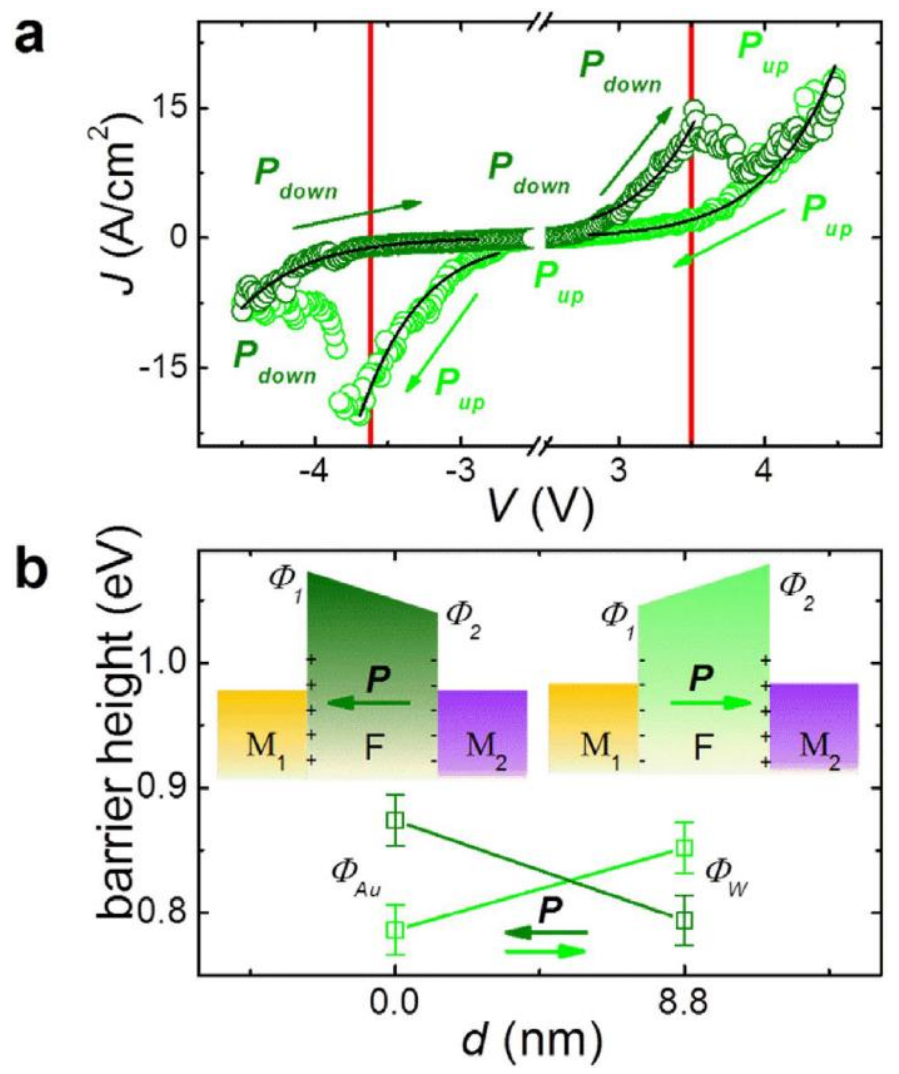

Figure 20. ER effect in a Si/Au/PVDF/W nanocapacitor. (a) Current density versus 
voltage $(J-V)$ curves of a $\mathrm{Si} / \mathrm{Au} / \mathrm{PVDF} / \mathrm{W}$ nanocapacitor. Solid lines are fits using the thermionic injection model. The arrows show the path of the current. The red lines indicate the coercive voltages from polarization switching. (b) Barrier heights statistically derived from thermionic injection (TI) fits as a function of the ferroelectric thickness $d$. The insets show a schematic representation of the energy potential profiles across the ultrathin ferroelectric PVDF films and the arrows denote the directions of the polarization. Reprinted from (Tian et al., 2015)
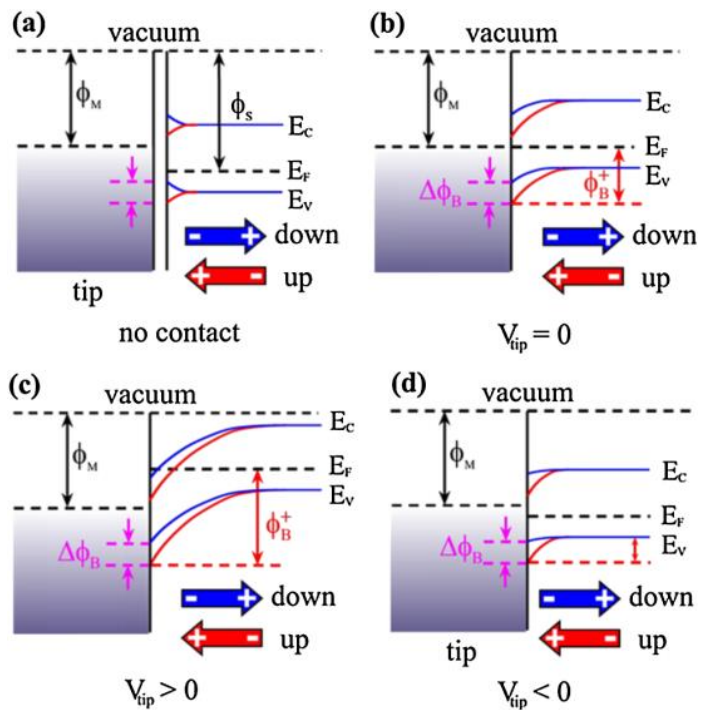

Fig. 21. Schematic interfacial band diagrams between an AFM tip and a ferroelectric material. (a) No contact between the tip (metal) and the sample (ferroelectric semiconductor). The presence of polarization bends the conduction (valence) band. (b) $V_{\text {tip }}=0$ (zero bias); (c) $V_{\text {tip }}>0$ (reverse bias); (d) $V_{\text {tip }}<0$ (forward bias). Reprinted from (Wu et al., 2010).

In the following, we incorporate equations (1)-(21) in a numerical simulator in order to analyze the effects of the modification of contact energetics by polarization. Complete details about modeling assumptions are provided in SI. A current-voltage curve for a symmetric ohmic MFM structure with no ferroelectric polarization in the dark is represented in Fig. 22a. As expected, the simulation leads to a linear relation between the current density and the applied voltage. When the ferroelectric layer is polarized, the energy barriers at the contacts are modified accordingly, see Fig. 22b. An effective barrier is developed with a rectifier behavior at the contact on the left when an equivalent volume charge density $\rho_{\text {pol }}=P / \delta$ is placed at a distance $\delta$ from the interface. A switchable behavior is obtained under the opposite polarization. Although the actual value of $V_{b i}$ is $V_{b i}=0$, an effective built-in voltage $V_{b i}{ }^{\prime}$ is created along the MFM structure (compare Fig. 22a' and b'). The effective $V_{b i}^{\prime}$ is shifted in the barriers with a value close to the order of $P \delta / \varepsilon_{0} \varepsilon_{r}$, as justified by the model of (Pintilie and 
Alexe, 2005). Fig. 22c shows the current-voltage curves under illumination. The loop around the origin is created when switching from positive to negative polarization. For the negatively poled samples, the short-circuit current and open-circuit voltage are positive and negative, respectively. After the positive poling, the photocurrent direction is reversed showing the switchable ferroelectric photovoltaic response.

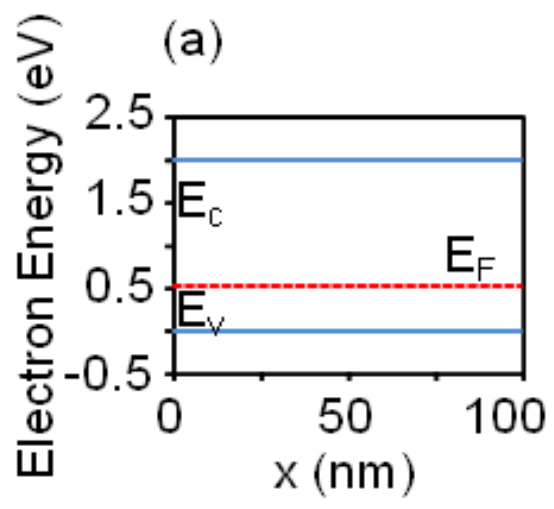

(d)

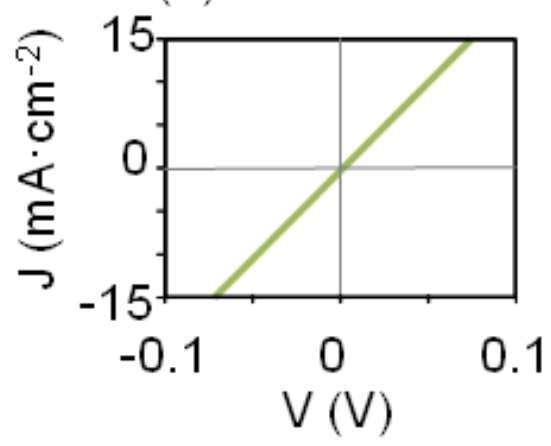

(b)

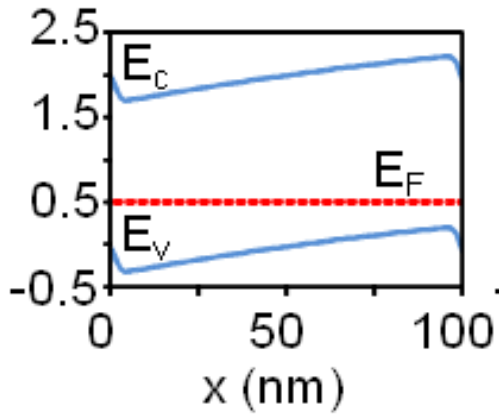

(e)

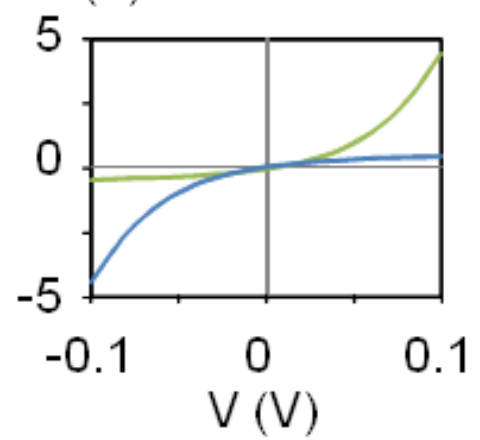

(c)

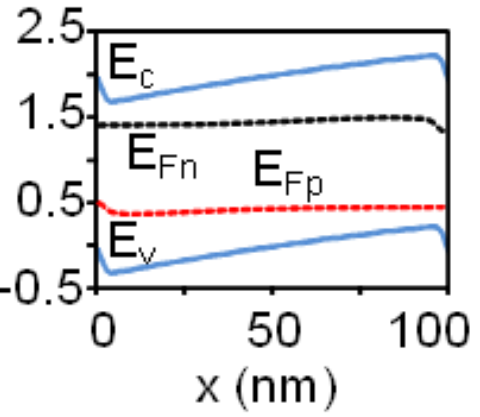

(f)

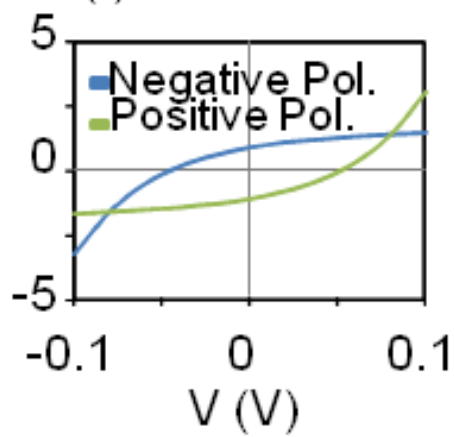

Fig. 22. Band Diagrams for a symmetric-ohmic MFM structure simulated under (a) no polarization in dark, (b) positive polarization in dark, and (c) positive polarization with illumination. (d)-(f) Corresponding current-voltage characteristics. The MFM is simulated using a doping concentration of $N_{A}=10^{17} \mathrm{~cm}^{-3}$, and an effective density of states $N_{C}=10^{20} \mathrm{~cm}^{-3}$, a layer thickness of $d=100 \mathrm{~nm}, \mu=1 \mathrm{~cm}^{2} / \mathrm{Vs}, \varepsilon_{\mathrm{r}}=50$ and $P_{\mathrm{r}}=6$ $\mu \mathrm{C} / \mathrm{cm}^{2}$. The ferroelectric effect is modeled with an effective localized charge at the interface related to the bound and screening charges.

As discussed before, the polarization of a ferroelectric layer can be stabilized with compensation charges, i.e. using conducting electrodes as in Fig. 18. The bound charge $P$ at the contact with the electrode, is compensated by the same total charge $-P$ at the electrode surface. The extent to which the charge is spatially distributed at the interface is an important feature for PV properties, as already mentioned in the model of (Pintilie and Alexe, 2005). Another important effect is that the charge in the conducting medium may spread some distance from the interface, according to the screening length of the electrode material, $l_{s}$. Then, $P$ is imperfectly screened, leading to a depolarization field 
(Batra et al., 1973,Mehta et al., 1973) with value

$$
F_{d p}=-\frac{P}{\varepsilon_{0} \varepsilon_{F}} \frac{\varepsilon_{F} l_{s}}{\varepsilon_{F} l_{s}+\varepsilon_{e} d / 2}=-\frac{P(1-\theta)}{\varepsilon_{0} \varepsilon_{F}}
$$

where $\varepsilon_{e}\left(\varepsilon_{F}\right)$ is the relative dielectric constant of the electrode (ferroelectric layer), and $\theta$ is the compensation ratio for polarization charge (Jo et al., 2006). In the case of metal contacts with different relative dielectric constants $\varepsilon_{e 1}$ and $\varepsilon_{e 2}$, the polarization field is (Zhuravlev et al., 2005) (Pantel and Alexe, 2010)

$$
F_{d p}=\frac{P}{\varepsilon_{0} \varepsilon_{F}} \frac{\varepsilon_{F}\left(\frac{l_{s 1}}{\varepsilon_{e 1}}+\frac{l_{s 2}}{\varepsilon_{e 2}}\right)}{\varepsilon_{F}\left(\frac{l_{s 1}}{\varepsilon_{e 1}}+\frac{l_{s 2}}{\varepsilon_{e 2}}\right)+d}
$$

where $l_{s 1}$ and $l_{s 2}$ are the respective screening lengths of the electrodes. Eq. (42) reduces to Eq. (41) when $\varepsilon_{e 1}=\varepsilon_{e 2}$ and $l_{s 1}=l_{s 2}$. The depolarization field always exists in ferroelectrics and is responsible for the instability of the spontaneous polarization. It becomes more important in the case of ultra-thin films (few nm thickness), when these films are fully depleted. This is an important effect in memory applications (Naber et al., 2010) and many studies indicate that the PV effect in ferroelectric materials is enhanced by the depolarization field which separates charge carriers (Brody, 1973,Masaaki et al., 2002,Ichiki et al., 2005, Qin et al., 2007). (Qin et al., 2009) showed that for a large dielectric constant material, the screening distance in the electrode material is considerably enhanced which produces larger depolarization field and an increase of PV performance.

\subsection{Schottky barrier models}

It has been widely recognized that the formation of Schottky barriers at the ferroelectric/semiconductor surface has a strong impact on PV properties. The modulation of the depletion width by the ferroelectric polarization was shown by (Blom et al., 1994). The state of polarization of the ferroelectric contact layer can control the electronic state of the semiconductor at the surface, from depletion to accumulation (Liu et al., 2013). For a polarization parallel to the built-in field, the depletion width is small and the resistance of the diode is low. Many publications have used a PV model based on the modification of the Schottky barriers, see Fig. 10, and demonstrated the control of the depletion regions by altering the polarization conditions (Yang et al., 2000,Yuan and Wang, 2009) (Guo et al., 2013,Fang et al., 2014). The model is also illustrated in Fig. 9c, and Fig. 13. For such cases, the current-voltage characteristic deviates from the classical diode like behavior and displays a hysteretic diode like trend, due to the hysteretic nature of the polarization itself, see Fig. 15. The large polarization charge detected at the $\mathrm{BiFeO}_{3} /$ metal interface significantly modifies the size of the Schottky barrier. Thus, for the upward polarization state, the $\mathrm{Pt} / \mathrm{BiFeO}_{3}$ and $\mathrm{BiFeO}_{3} / \mathrm{SrRuO}_{3}$ interfaces can have blocking and nearly non-blocking contacts, respectively, as shown in 
Fig. 13a. For downward polarization the role of the barriers is reversed (Lee et al., 2011).

The characteristics of voltage switching have been amply described in the literature of ferroelectrics. The polarization of a symmetric $\mathrm{Au} / \mathrm{BFO} / \mathrm{Au}$ sample by (Yi et al., 2011) produces diode characteristics that are completely switchable under reversal of polarization. The inversion of the diode characteristics depends heavily on the poling temperature. The rectifying characteristics are explained in terms of a reduction of the barrier height by the polarization charge that converts the initial Schottky barrier to an ohmic contact, as mentioned earlier. The migration of positively charged oxygen vacancies is another effect that often plays a dominant role. The migration of vacancies modifies the number of defects in the depletion region and therefore the spatial extent of the depletion, which influences the charge collection effectiveness of the barrier under illumination (Yang et al., 2016). It is suggested that migration of oxygen vacancies is a primary mechanism for the switchable PV effect (Yi et al., 2011) (Cao et al., 2014).

In general, the height of a Schottky barrier does not correspond straightforwardly to the difference of the work functions between the metal and the semiconductor as in Eq. (31). The interface states pin the Fermi level and the dependence of the Schottky barrier height on the metal work function is described by a parameter $S$, which can be estimated from the optical dielectric constant or using the model of metal induced gap states (MIGS). For $\mathrm{BiFeO}_{3}$ the value $S=0.23$ has been obtained, so that the barrier of Pt will be $0.95 \mathrm{eV}$ and the barrier to a typical conducting oxide would be $0.9 \mathrm{eV}$ (Clark and Robertson, 2007).

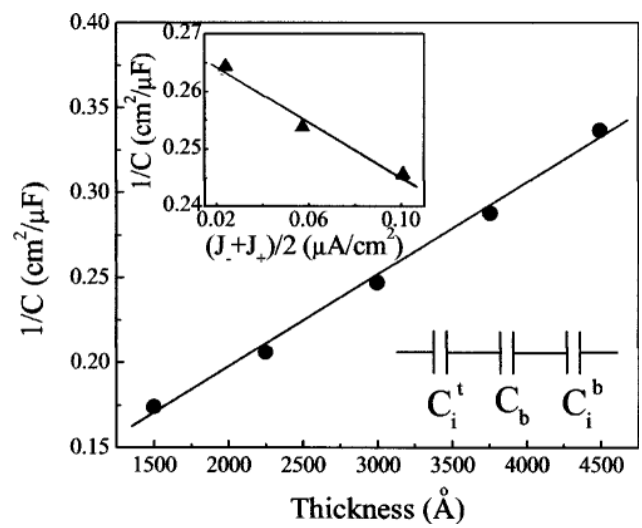

Fig. 23. Reciprocal capacitance vs. film thickness of $\mathrm{PbZr}_{0.53} \mathrm{Ti}_{0.47} \mathrm{O}_{3}$ (PZT) 300-nmthick film capacitors of symmetric structure Pt/PZT/Pt. Reproduced from (Yang et al., 2000).

An important approach to the analysis of ferroelectric layers including Schottky barrier studies is the measurement of capacitance. (Yang et al., 2000) have measured the dependence of the capacitance on the ferroelectric thickness $d$ and they found that the capacitance contains two components, one is the bulk layer capacitance $\left(\varepsilon_{r} \varepsilon_{0} / d\right)$ and 
the other one is the interfacial capacitance $C_{i}$. Fig. 23 shows a linear dependence of the reciprocal capacitance on the film thickness according to the expression

$$
\frac{1}{C_{t o t}}=\frac{d}{\varepsilon_{r} \varepsilon_{0}}+\frac{1}{C_{i}}
$$

The interfacial capacitance obtained from the interlayer is $11.3 \mathrm{mF} / \mathrm{cm}^{2}$. The value obtained for the bulk dielectric constant is $\varepsilon_{r}=1400$. These results (Yang et al., 2000) have been interpreted in terms of the internal field $\left(F_{i}\right)$, caused by defects and vacancies, and the depolarization field $\left(F_{d p}\right)$ that coexisted in the PZT film. (Pintilie et al., 2007) remarked that there are two main contributions to the voltage dependence of the capacitance. One is the variation of the depletion region, and another is the dependence of polarization on the voltage, in the case that it is not saturated. Therefore one needs to check the domain of variation of capacitance in relation with $P-F$ hysteresis curve before adopting a sound interpretation.

In the method of (Zheng et al., 2008), two separate contributions to the photocurrent are assumed, the depolarization field, $F_{d p}$, and the field at the contact barriers, $F_{i b}$. Thus, a net built-in field at the barriers is $F_{b i}=F_{i b-b o t t o m}-F_{i b-t o p}$. If the barriers are nearly similar but the depolarization field is reversed under a switch of the remnant polarization, then the photocurrent due to each contribution is given by the expressions

$$
\begin{aligned}
& J_{i b}=\frac{J_{u p}+J_{\text {down }}}{2} \\
& J_{d p}=\frac{J_{u p}-J_{\text {down }}}{2}
\end{aligned}
$$

where $J_{u p}$ and $J_{\text {down }}$ are the photocurrents close to the poled state. This method has been widely applied with different variants. (Cao et al., 2011) found that the photocurrent increases with increasing annealing time, which is ascribed to a removal of defects and a decrease of the leakage current. In these measurements, the depolarization field of the alignment polarization has the main influence on the photocurrent.

A series of publications from the 1960's and 1970's (Neumark, 1962,Mehta, 1972,Brody and Crowne, 1975,Heyszenau, 1978) tackled modeling of the effect of ferroelectric polarization on the voltage drop at the ferroelectric/photoconductor barrier. The authors could therefore study the influence of a switch of polarization on the PV performances, usually at short-circuit. Using numerical simulations, we now analyze in more details the Schottky solar cell shown in Fig. 6, including the effective polarization charge density at the ferroelectric-metal interface. In the model, we assume an asymmetric MFM structure with an ohmic contact on one side and a rectifier contact on the other side. We solve the Poisson equation in the semiconductor material coupled to the continuity equation that includes drift, diffusion and recombination processes. The polarization charges, including the screening effects, are modelled with fixed effective charges of opposite sign located at a distance $\delta$ from both metal-ferroelectric interfaces. They work as an effective dipole. 
As shown in Fig. 24, the ferroelectric polarization affects the short-circuit current density and the open-circuit voltage in opposite ways. In the case of the negative (positive) poling the effective built-in voltage is higher (lower) than the initial built-in voltage. A higher built-in voltage produces a higher open-circuit voltage. Thus, for negative poling the $V_{\mathrm{oc}}$ is higher than the original $V_{\mathrm{oc}}$. The opposite trend is observed with the value of $J_{\mathrm{sc}}$. For negative poling, the injection barrier decreases but the barrier at the extraction electrode increases which hinders the removal of the charge and $J_{\mathrm{sc}}$ decreases. In this asymmetric device, no switching behavior is obtained. The positive and negative poling simply modify the degree of asymmetry between the two contacts (a scheme of this idea is displayed in the right column of Fig. 10a,b,c). After the modification of the barrier heights with poling, the rectifier and ohmic contacts still play the same role as rectifier and ohmic contacts, respectively.

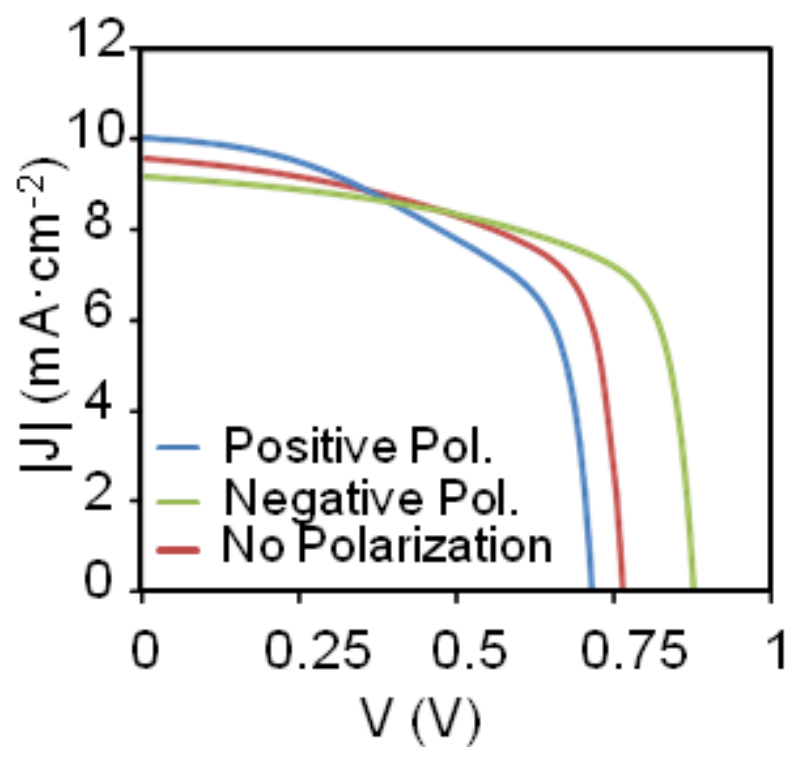

Fig. 24. Effect of the polarization charge at metal-ferroelectric interface: Currentvoltage characteristic in an asymmetric MFM structure under illumination with positive and negative poling and without poling.

In order to observe a switching behavior in the $J-V$ curves a symmetric structure must be considered. The band diagram of a symmetric MFM structure with Schottky contacts and no ferroelectric polarization under dark condition at zero applied voltage is represented in Fig. 25a. In this case, the current density both at positive and negative bias voltages is limited by the saturation current of the Schottky diode, Fig. 25b. Under positive or negative polarization, the energy barriers are modified, and subsequently, the symmetry of the $J-V$ curve is broken. One contact plays the role of a rectifier contact and the other one acts as ohmic contact (see the left column of Fig. 10a,b,c for illustration). The $J$ - $V$ curves for these two poling cases are shown in Fig. 25c. When the 
MFM is illuminated, the $J-V$ curves show a hysteresis loop as depicted in Fig. 25d.

We conclude here that in a ferroelectric semiconductor device with ideal contacts, the polarization charge would be totally screened and no PV effect at all would exist. However, real materials show a depolarization field, smooth termination of polarization, and other inhomogeneous features as Schottky barriers that do provide the control of interface and bulk electric field by switchable spontaneous polarization.

(a)

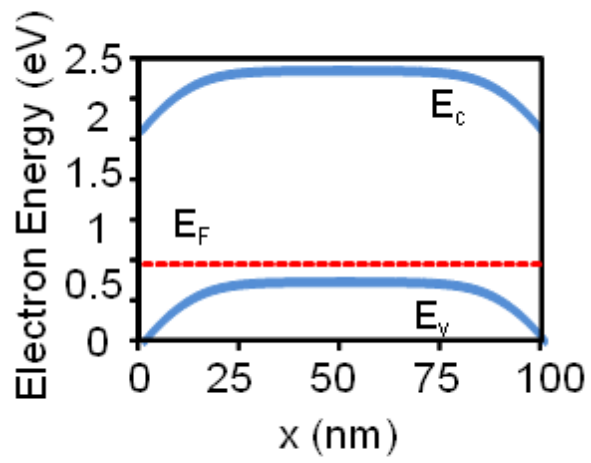

(c)

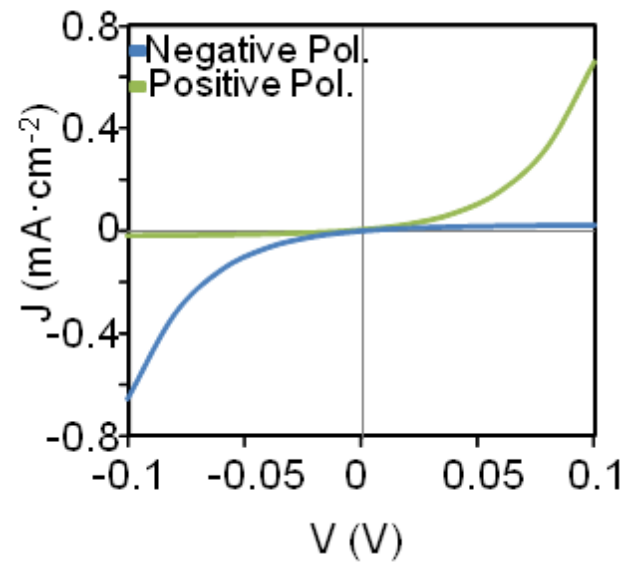

(b)

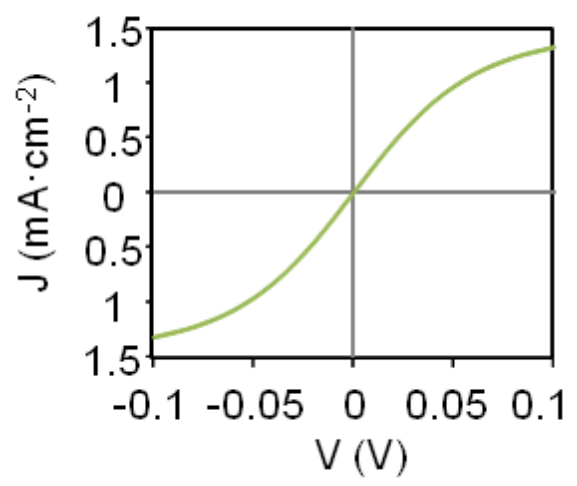

(d)

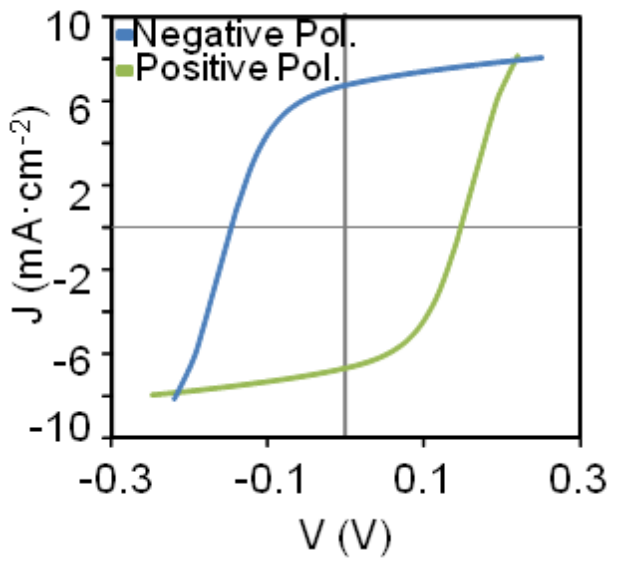

Fig. 25. (a) Electron energy scheme of a symmetric Schottky MFM structure with the following parameters of the ferroelectric semiconductor: doping concentration of $N_{A}=10^{17} \mathrm{~cm}^{-3}$, film thickness of $d=100 \mathrm{~nm}, \mu=1 \mathrm{~cm}^{2} / \mathrm{Vs}$ and $\varepsilon_{\mathrm{r}}=10$. The height of the energy barriers at the contacts are con sidered to be $0.8 \mathrm{eV}$. (b) Current-voltage characteristic for this MFM structure in dark with no poling effects. The semiconductor is considered ferroelectric (c), (d) Current-voltage characteristics in the MFM structure under positive and negative (c) polarization without and (d) with illumination.

One should realize that a large part of the reported ferroelectric solar cells have so far been dominated by Schottky barrier effects, while in planar geometry, when the size of the absorber is in the range from several micrometers to much larger (depending on the 
semiconductor optical absorption coefficient) in order to harvest completely the solar spectrum, then the interfaces cease to play any role in deciding the PV properties. However, in practice to grow micron to more thick film is quite challenging. To the best of our knowledge there is no systematic study on the thickness dependence of the PV effect to discriminate from interface and bulk effects. This seems a potential very interesting research to be done in the future.

\subsection{Spatially dependent polarization}

The effect of considering the hysteresis $P-F$ cycles as the switching between two constant values of the polarization was treated in the previous section. In that case, a constant value of the polarization along the ferroelectric with discontinuities at the contacts was modeled with the effective surface charge at the interfaces. In this section, we analyze the effect of considering the hysteresis $P-F$ cycles as the switching between two polarization states in which $P=P(F) \neq$ constant.

The relation between the applied voltage and polarization in a ferroelectric material is obtained by minimizing the total free energy of the system, $W$, which contains the contributions from the lattice, $W_{l a t}$, and the electronic contribution, $W_{e l}$. There are different approaches to model the polarization-field $(P-F)$ relation. The models based on Landau and Devonshire theories (Smith, 2001,Chandra, 2006), make use of the relation between the free energy $W$ with the polarization $W(P)$ and relate the electric field and the polarization as $F=\mathrm{d} W(P) / \mathrm{d} P$ (Smith, 2001, Suryanarayana and Bhattacharya, 2012). An approximation of this model is based on the hyperbolic tangent (tanh) function (Smith, 2001). (Miller et al., 1990,Miller et al., 1991,Reece and Ducharme, 2009) also interpreted the device characteristics for ferroelectric devices based on a tanh-function to reproduce the ferroelectric polarization hysteresis. Finally, Preisach's model includes a history-dependent electric field effect (Preisach, 1935,Yang et al., 2009). The widely used tanh-function (Blom et al., 1994,Liu et al., 2004,Reece and Ducharme, 2009) is given by the expression (shown in Fig. S2 in SI)

$$
P(F)=\varepsilon_{0}\left(\varepsilon_{r \infty}-1\right) F \pm P_{r} \tanh \left(\frac{F_{c} \pm F}{F_{c}}\right)
$$

where $P_{r}$ is the remnant polarization and $F_{\mathrm{c}}$ is the coercive electric field. Eq. (46) is an approximation that considers that the intrinsic polarization in a sample is the superposition of the polarization of all the elementary dipoles (Liu et al., 2004). This approach is simple but it models quite accurately the ferroelectric effect on the performance of ferroelectric solar cells. Combining Eqs. (14) and (46) the relative permittivity is:

$$
\varepsilon_{r}=\varepsilon_{r \infty} \pm \frac{P_{r}}{\varepsilon_{0} F_{c}} \cosh ^{-2}\left(\frac{F_{c} \pm F}{F_{c}}\right)
$$

where the positive sign corresponds to the forward scan and the negative one to the reverse scan. 
To assess the effect of ferroelectric polarization on the PV performance of solar cells, we apply the previous modeling equations to the Schottky barrier solar cell studied in Fig. 6.
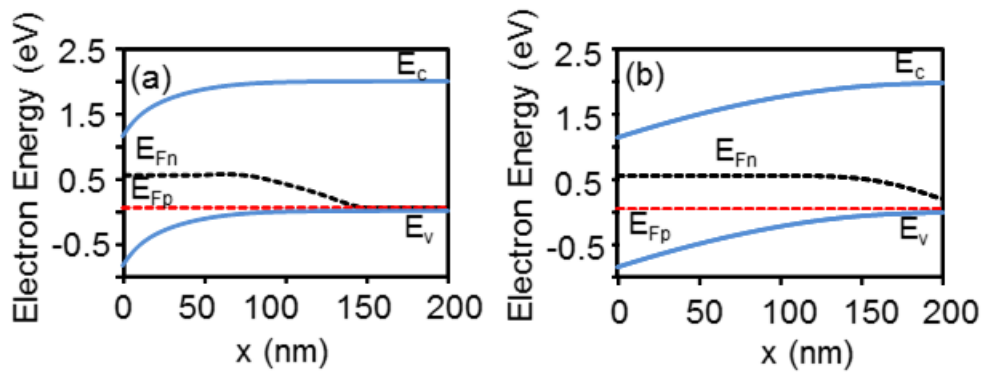

Fig. 26. Energy diagram along the device of Figure 6 and parameters defined in Table I (SI) for an applied voltage of $0.5 \mathrm{~V}$ (during the forward scan) and $N_{\mathrm{A}}=10^{18} \mathrm{~cm}^{-3}$ (a) without polarization and (b) with polarization. In this example, the device length is $L=$ $200 \mathrm{~nm}$ and is biased at $0.5 \mathrm{~V}$.

In order to isolate the effect of this phenomenon, the effect of the polarization charges is neglected in a first step, but is incorporated subsequently. Fig. 26 represents the energy diagrams along the device without polarization (a) and with polarization in (b), both for a forward scan. The inclusion of the ferroelectric polarization (44) in the Poisson equation (13) leads to a significant modification in the depletion region of a semiconductor, as shown in Fig. 26. In ferroelectric materials, the existence of ferroelectric polarization decreases the total density of charge $\rho_{\mathrm{T}}=\rho / \varepsilon_{\mathrm{r}}$, as inferred from section 2, Eqs. (9) and (13). Thus, the polarization induces a deeper penetration of the electric field within the bulk of the semiconductor and the depletion width increases. According to Eq. (14) a large dielectric constant can be obtained at electric fields close to the coercive field $F_{\mathrm{c}}$, where the slope is very large. Around this value of the electric field, a large depletion region is obtained. The increment of the depletion region with the polarization makes the length and doping of the active layer to play an important role in the device characteristics.

In the last two sections, 4.2 and 4.3, we have studied separately the contribution of two ferroelectric effects on the performance of MFM solar cells: the polarization charges at the interfaces, which modify the effective injection barriers, and the spatially dependent polarization (or electric field dependent permittivity), which modifies the extension of the effective depletion region in the semiconductor. Now, we study both effects simultaneously in different MFM structures. In an asymmetric MFM solar cell, none of both effects, separately (Fig. 24) or simultaneously (Fig. 27a) allow a switching behavior in the $J-V$ curves. The reason is the initial strong asymmetry of the structure. The existence of negative or positive poling only modifies the value of the effective built-in voltage (high value without polarization), and yet it is not enough to create a switchable behavior (see right column of Fig. 10a,b,c). 
In Figs $27 b$ and 27c, we include both effects in the simulation of symmetric-ohmic and symmetric-Schottky MFM diodes. The symmetric-Schottky MFM diode shows a noticeable hysteresis loop in the $J-V$ curves, Fig. 27b, as observed experimentally in the literature. In the case of ohmic-symmetric MFM diodes, surface polarization charges create a small rectifier barrier at one of the contacts. Nevertheless, its effect is diminished by the bulk effects associated to the electric field dependent permittivity in the bulk reducing the hysteresis loop, Fig. 27c.

As a first conclusion, symmetric Schottky MFM structures are thus necessary to obtain a noticeable switchable behavior or a large hysteresis loop in the $J$ - $V$ curves under illumination. A second conclusion is that both effects associated to the ferroelectric polarization (surface polarization charges or bulk polarization charges) are necessary in order to interpret experimental $J-V$ curves obtained so far.
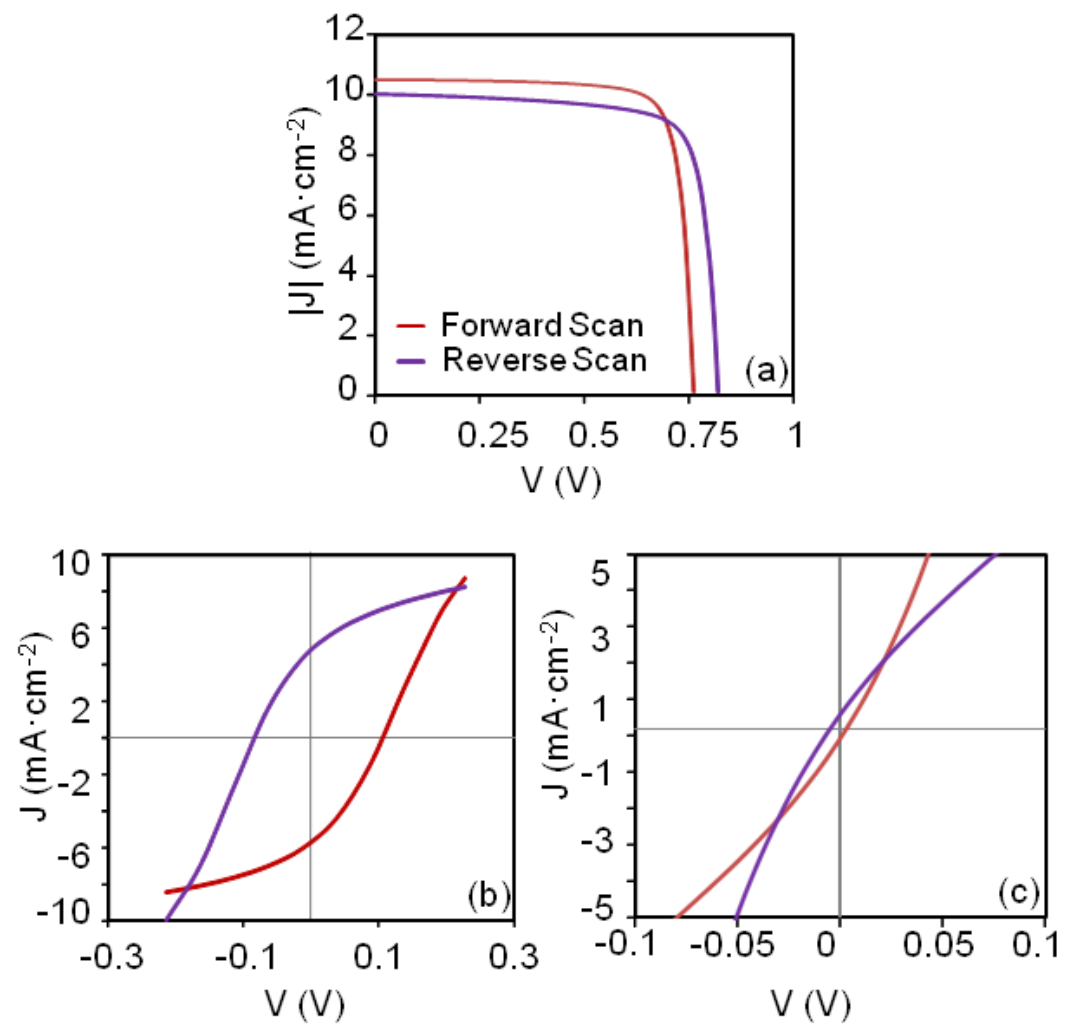

Fig. 27. Combined effect of the spatially dependent polarization and the polarization charge density at the MF interface on the performance of MFM solar cells, under illumination with positive and negative poling. Current-voltage characteristic in an asymmetric (a) symmetric-Schottky (b) and symmetric-ohmic (c) MFM structure.

\section{The bulk photovoltaic effect}

As already discussed in Section 2, all commercial and most laboratory solar cells achieve charge separation and directionality of current flow by using either a built-in 
voltage (for instance in a $p$ - $n$-junction, Schottky-junction or $p$ - $i$ - $n$ junction) or another type of energy barrier that results in contacts that mostly inject and extract one charge carrier and not the other. This conventional way of operating a solar cell is sometimes called the barrier photovoltaic effect and is in contrast to the more rarely observed and used photogalvanic or bulk photovoltaic effect. In a normal solar cell, any electron and hole current densities, $J_{\mathrm{n}}$ and $J_{\mathrm{p}}$, are driven by a gradient of the electron and hole Fermi levels as stated in Eq. (19) and (20). Thus, at open circuit the requirement $J=0$ leads to flat quasi-Fermi levels for electrons and holes as discussed before. The drift current is therefore perfectly balanced by the diffusion current. In the case of the 'normal' barrier photovoltaic effect, the $q V_{\mathrm{oc}}$ is equal to the quasi-Fermi level splitting, Eq. (3), which cannot easily exceed the band gap.

In non-centrosymmetric materials, such as ferroelectrics, this is no longer the case as shown in Fig. 28. Here, under non-equilibrium illumination, charge carriers are created with a built-in momentum (Glass et al., 1974,Belinicher, 1980,Von Baltz and Kraut, 1981,Sturman and Fridkin, 1992, Young et al., 2012,Zenkevich et al., 2014,Wang and Rappe, 2015,Zheng et al., 2015) that creates a directed photogalvanic current. The asymmetric charge generation creates so-called shift currents (labeled as $J_{\text {shift }}$ in Fig. 28) without the need to have a gradient in the electrochemical potential (Ruppel et al., 1982). Open circuit is reached, the shift current is balanced by a drift current and therefore the quasi-Fermi levels are no longer flat at open circuit. There exist various models in the literature which attribute the causes for an asymmetric momentum of the photogenerated charge carriers to impurities or scattering centers. One example, an asymmetric potential well, is shown in Fig. 29. Other examples that could lead to a built-in momentum of photogenerated charge carriers would be asymmetric scattering centers or a shift in the conduction band or valence band in k-space due to spin-orbit coupling (Belinicher, 1980,Butler et al., 2015).

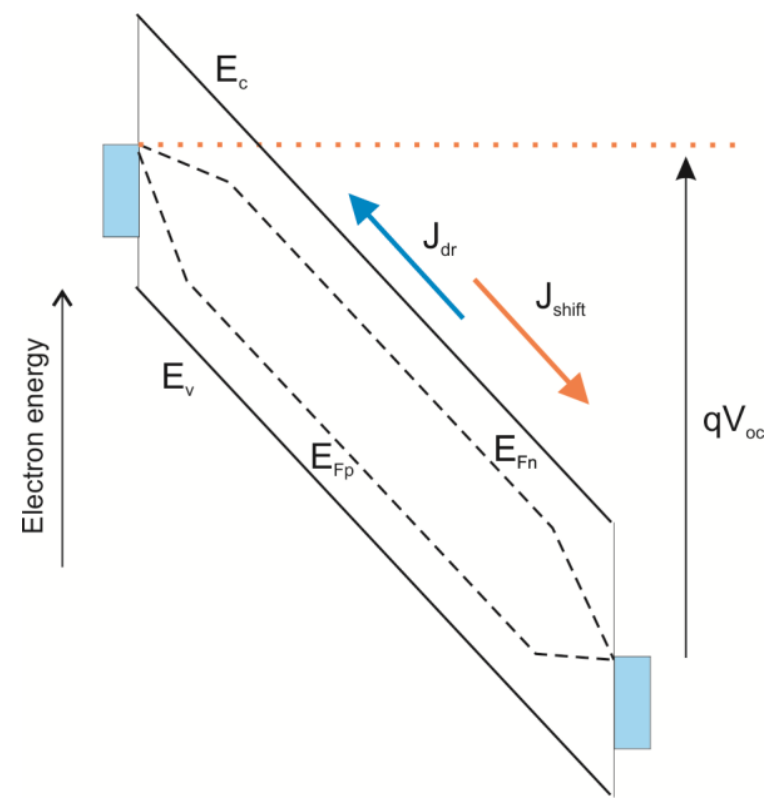


Fig. 28. Schematic band diagram illustrating the bulk-photovoltaic effect at open circuit. No built-in voltage is necessary for carrier separation. The quasi-Fermi levels do not have to be flat at open circuit and $q V_{\mathrm{oc}}$ can greatly exceed the band gap $E_{\mathrm{g}}$ if the device is thick enough.

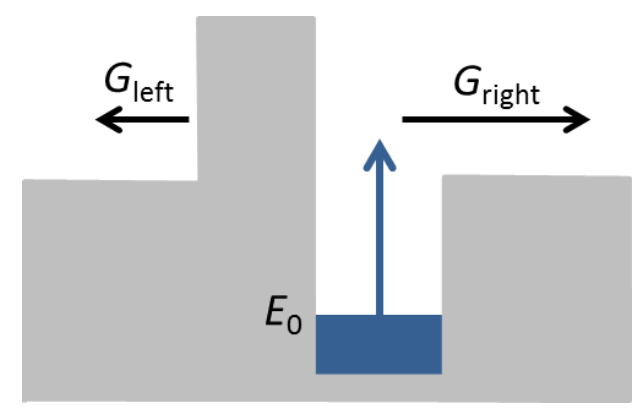

Fig. 29. Example of an asymmetric potential well illustrating one microscopic mechanism that could cause the bulk-photovoltaic effect. In this case, an asymmetric potential well leads to an asymmetry in momentum for the photogenerated carriers. Adapted from (Ruppel et al., 1982).

The short-circuit current density due to the bulk-photovoltaic effect under monochromatic illumination is often expressed using the so-called Glass constant $\kappa$ as (Ruppel et al., 1982,Zenkevich et al., 2014)

$$
J_{\mathrm{sc}}=\kappa \alpha P_{\mathrm{opt}}=\kappa \alpha(h v) \Phi_{\mathrm{opt}}
$$

where $\alpha$ is the absorption coefficient, $P_{\text {opt }}$ is the optical power density in W/cm ${ }^{2} h v$ is the photon energy and $\Phi_{\mathrm{opt}}$ is the photon flux density in $\mathrm{cm}^{-2} \mathrm{~s}^{-1}$. While the Glass constant $\kappa$ with unit $\mathrm{cm} / \mathrm{V}$ is described here as a scalar as in the original publication by Glass et al., it is in fact a component of a third rank tensor. We refer the interested reader to the book by (Sturman and Fridkin, 1992), cf. Eqs. (1.27) and (1.28), or to (Young and Rappe, 2012,Young et al., 2012) for more in-depth description of the photocurrent and the Glass coefficient.

There are two possible microscopic mechanisms of the bulk photovoltaic effect, typically called 'ballistic' and 'shift' currents (Sturman and Fridkin, 1992,Zenkevich et al., 2014). In case of the ballistic mechanism, photogenerated carriers are generated with a built-in momentum that allows them to move the mean free path $l_{0}$ before thermalizing to the band edge. In case of the 'shift' mechanism, the carriers are virtually shifted in real space by the shift vector with amplitude $R$. The mean free path is estimated to be on the order of 10 to $100 \mathrm{~nm}$ (Sturman and Fridkin, 1992). Theoretical calculations showed that the amplitude $R$ of the shift vector is typically only in the range of a few $\AA$ to a few nm (Young and Rappe, 2012).

In the following, we will follow the description of (Zenkevich et al., 2014) describing the ballistic case. The Glass coefficient can then be expressed as 


$$
\kappa=q l_{0} \xi \frac{\varphi}{h v}
$$

where, $l_{0}$ is the mean free path and $\xi$ is a measure of the excitation asymmetry and $\varphi$ is the quantum yield for charge generation. This allows us to rewrite the short-circuit current density to

$$
J_{\mathrm{sc}}=q l_{0} \xi \varphi \alpha \Phi_{\mathrm{opt}}
$$

In order to generate a photocurrent several photons are needed to bridge the distance between the contacts by numerous cases of photogeneration with an associated movement of carriers by the distance $l_{0}$ followed by recombination and excitation with another photon. Thus, for devices where the distance $d$ between the electrodes is much larger than $l_{0}$ (or much larger than the shift vector $R$ depending on which mechanism dominates) photocurrents will be comparably low.

At open circuit, the total current must vanish and the drift current $J_{\mathrm{dr}}=\sigma F$ must compensate the shift current. Thus, the open-circuit voltage is given by

$$
V_{\mathrm{oc}}=\frac{J_{\mathrm{sc}} d}{\sigma}=\frac{q l_{0} \xi \varphi \alpha \Phi_{\mathrm{opt}} d}{\sigma_{\mathrm{d}}+\sigma_{p h}}
$$

Here, the conductivity $\sigma$ is split up as indicated in Eq. (34). If we further assume that the photoconductivity exceeds the dark conductivity such that we can write $\sigma \approx \sigma_{p h}$ and if we can neglect any spatial dependence of the optical generation rate and the excess electron concentration $\Delta n$, we can write

$$
\sigma_{\mathrm{ph}}=q\left(\mu_{\mathrm{n}}+\mu_{\mathrm{p}}\right) \Delta n=q\left(\mu_{\mathrm{n}}+\mu_{\mathrm{p}}\right) \alpha \varphi \Phi_{\mathrm{opt}} \tau
$$

where $\tau$ is the lifetime of the charge carriers. Thus, we obtain for the open-circuit voltage

$$
V_{\mathrm{oc}}=\frac{q l_{0} \xi \alpha \varphi \Phi_{\mathrm{opt}} d}{q\left(\mu_{\mathrm{n}}+\mu_{\mathrm{p}}\right) \alpha \varphi \Phi_{\mathrm{opt}} \tau}=\frac{l_{0} \xi d}{\left(\mu_{\mathrm{n}}+\mu_{\mathrm{p}}\right) \tau}
$$

Therefore, the open-circuit voltage, in case of the bulk-photovoltaic effect, has some rather peculiar properties that are different to those of the barrier-photovoltaic effect. If the distance $d$ between the contacts is made high enough, there is nothing that prevents the open-circuit voltage from exceeding the band gap several times (Yang et al., 2010,Bhatnagar et al., 2013). To observe these above-gap voltages, it is therefore useful to make coplanar devices, where the electrode distance is on the order of 10 to $100 \mu \mathrm{m}$ (Yang et al., 2010,Bhatnagar et al., 2013). In addition, a high mobility-lifetime product (at constant mean-free path) would decrease the open-circuit voltage because of the decreased resistance of the device that is needed to build up a photovoltage in the absence of any built-in voltage or other charge separating macroscopic barrier. Once the light intensity is sufficiently high that the photoconductivity exceeds the dark conductivity, the open-circuit voltage does not depend on light intensity anymore (Zenkevich et al., 2014). Any increase in light intensity would both increase the short- 
circuit current density and the photoconductivity at the same rate and therefore lead to zero extra open-circuit voltage. Experimental results by (Bhatnagar et al., 2013) show that the open-circuit voltage for the bulk photovoltaic effect increases strongly when the temperature is reduced. This increase in $V_{\text {oc }}$ with a decrease in temperature is much stronger than the linear effect usually observed in non-ferroelectric solar cells. In the case of $\mathrm{BiFeO}_{3}$, the $V_{\text {oc }}$ increases nearly exponentially with decreasing temperature due to the drop in conductivity of the material at lower temperatures (Bhatnagar et al., 2013).

If voltage is applied parallel to the direction of the polarization, the photocurrent depends on voltage in a very similar fashion to Eq. (35):

$$
J=J_{s c}-\left(\sigma_{d}+\sigma_{p h}\right) \frac{V}{L}
$$

In this case, the current-voltage characteristic is also linear. This effect is also claimed as an origin of the domain wall effect discussed below (Bhatnagar et al., 2013). However, the dependence on the polarization direction for the bulk photovoltaic effect and the barrier effect is different.

Based on the short-circuit current density, the open-circuit voltage and the linear current-voltage curve, we can calculate an efficiency for the bulk-photovoltaic effect. The efficiency $\eta$ of a solar cell is in general given by Eq. (24). The fill factor $F F$ is equal to $25 \%$ due to the linear current voltage curve. Using Eqs. (24), (48) and (51), we obtain

$$
\eta=\frac{q \alpha \varphi\left(l_{0} \xi\right)^{2} d}{4\left(\mu_{\mathrm{n}}+\mu_{\mathrm{p}}\right) \tau h v}
$$

The bulk-photovoltaic effect does currently not lead to high photovoltaic efficiencies. This is due to the fact that the mean free path $l_{0}$ is typically not high enough to allow high photocurrent densities. To maximize the photocurrents obtained from the bulkphotovoltaic effect one would have to use thin-films with sandwich-type contacts like in conventional thin-film solar cells. This has been done recently by (Zenkevich et al., 2014). However, the reported power conversion efficiencies were below $1 \%$. In such a thin-film configuration, high open-circuit voltages are impossible because the requirement of high electrode distance to achieve a high $V_{\mathrm{oc}}$ competes with the necessity of keeping the thickness close to the mean free path to achieve a high $J_{\text {sc }}$.

In principle it is conceivable to improve classical PV devices using the barrier photovoltaic mechanism by using ferroelectric materials that have additional shift currents. However, the effect might be small in reality because the requirement of a low photoconductivity in order to achieve high open-circuit voltages using the bulkphotovoltaic effect collides with the requirement for high mobility-lifetime products that is crucial for classical photovoltaics.

It is also known from experiments that observation of high photovoltages in ferroelectric materials depends strongly on the orientation of the ferroelectric domain 
walls relative to the contacts. While earlier papers suggested that the domain walls are responsible for the charge separation mechanism (Yang et al., 2010), the work of (Bhatnagar et al., 2013) showed that the bulk photovoltaic effect can be observed, in principle, independent of the orientation of the domain walls. However, the domain walls increase the conductivity of the system if the contacts are perpendicular to the domain walls. Thus, in this configuration the achievable open-circuit voltages are reduced relative to the case when domain walls and contacts are parallel to each other (see Fig. 30 for an illustration of the different measurement geometries).

A similar behavior is found in the domain wall effect suggested by (Seidel et al., 2011). This case corresponds to an alternative succession of domains where photogenerated electrons and holes strongly recombine, and domain walls where electrons and holes are separated and build up at the domain wall interfaces. In this case, illumination and voltage also contribute to vary the voltage drop in the domain wall and the current-voltage characteristic is also linear.

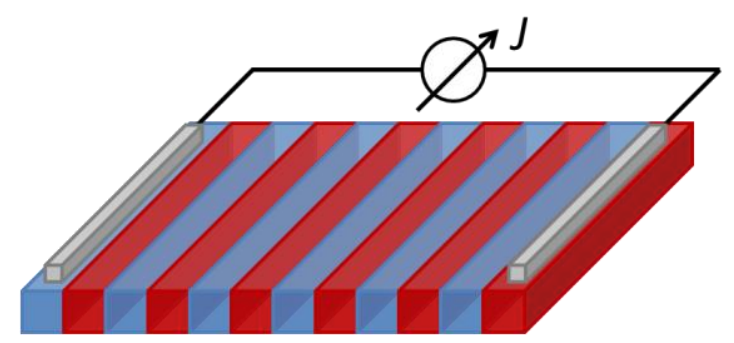

(a) contacts parallel to domain walls

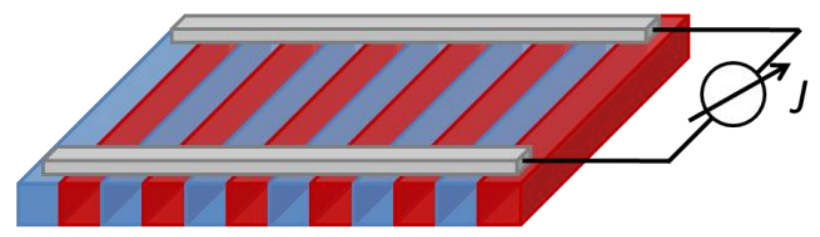

(b) contacts perpendicular to domain walls

Fig. 30. Schematic of two different measurement geometries with coplanar contacts. These measurement geometries are well suited to observe large open-circuit voltages exceeding the band gap, because they allow the distance between the contacts to be made large as compared to the film thickness. If the contacts are parallel to the domain walls (a) typically larger open-circuit voltages are measured while the increased conductivity of the domain walls e.g. in $\mathrm{BiFeO}_{3}$ leads to reduced open-circuit voltages if as shown in (b) the contacts are perpendicular to the domain walls.

\section{Energy barrier at interfaces}

A ferroelectric layer has the advantage that it can produce a very large electric field in a thin spatial layer that may offset the energy levels inside a PV device. Here we 
consider the introduction of an additional surface field formed by a thin ferroelectric layer that assists the properties of another semiconductor absorber material. Introducing an additional electrical field in OPV devices by polarized ferroelectric films provides a tool to enhance the solar cell efficiency, as first reported by (Yuan et al., 2011). When the dielectric layer is poled, it obtains a permanent field that displaces the built-in field in the charge collection layer as shown in Fig. 31. If the system is polarized in reverse then the built-in field increases. Therefore, the ferroelectric layer can enhance exciton separation and long range transport to improve charge collection (Kirchartz et al., 2015). Another important effect that can be formed by a ferroelectric layer is to improve the efficiency of charge injection at contact barriers.

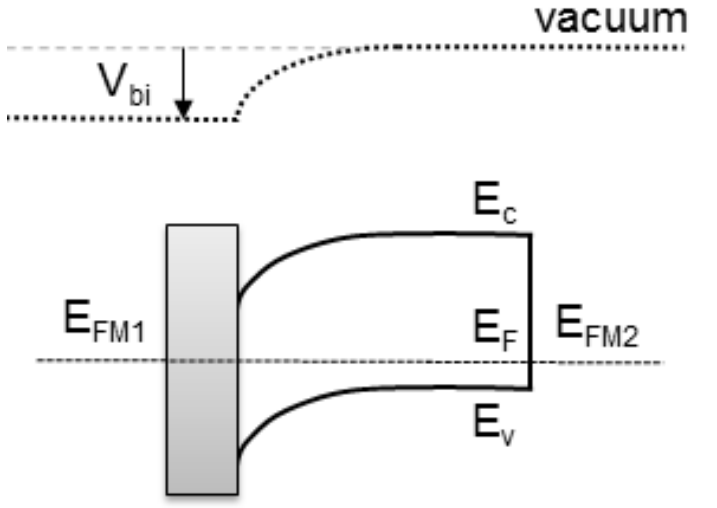

Metal 1 FE Semiconductor Metal 2

(a)

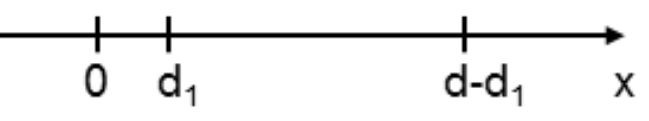

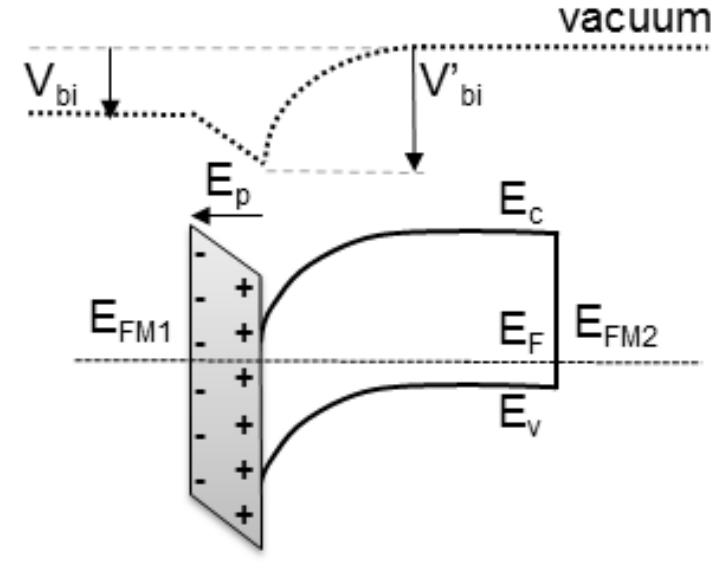

Metal 1 FE Semiconductor Metal 2 (b)

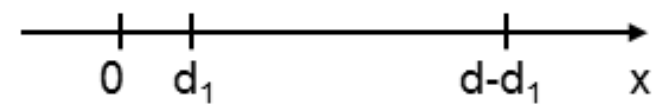

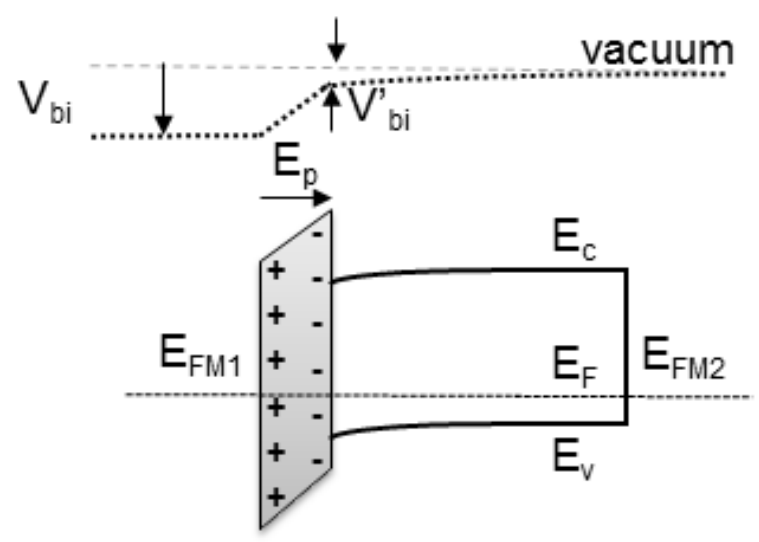

Metal 1 FE Semiconductor Metal 2

(c)

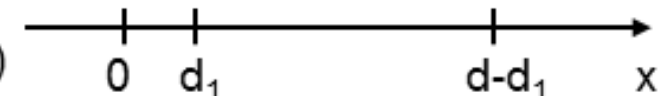

Fig. 31. Band diagrams of MFSM photovoltaic structure under non-poling, positive and negative poling, (a), (b) and (c), respectively. The introduction and polarization of the ferroelectric layer allows for a modification of the effective built-in potential $V_{b i}^{\prime}$ in 
the semiconductor. Its value can be higher or lower than the nominal built-in potential depending on the polarization.

In the case of positive poling, the effective built-in voltage in the semiconductor is higher than the built-in voltage. However, there are other aspects of the ferroelectric layer that must be carefully balanced. In Fig. 31b, the layer increases the built-in voltage for charge collection but it forms an energy barrier that opposes the carrier extraction. In addition, the surface ferroelectric layer introduces a series resistance. Another potential problem in the setup of Fig. 31 is that the bound polarization charge at the ferroelectric semiconductor interface cannot be adequately compensated in the case of low conductivity or depletion in the semiconductor. This will lead to a large uncompensated field in the ferroelectric layer that will remove the polarization charge (Asadi et al., 2011).

The first observation of enhanced PV performance was for a thin ferroelectric polymer $\mathrm{P}(\mathrm{VDF}-\mathrm{TrFE})$ inserted between an organic bulk heterojunction photoactive layer P3HT:PCBM (poly(3-hexylthiophene): phenyl-C ${ }_{61}$ butyric acid methyl ester) and the electrode (Yuan et al., 2011). When all of the dipoles in the two-dimensional $\mathrm{P}(\mathrm{VDF}-\mathrm{TrFE})$ layer are aligned by an external electrical field, two sheets of net charges form at each side of the P(VDF-TrFE) film as indicated in Fig. 31b. Since the P(VDFTrFE) layer is directly in contact with the metal electrode, the charge is partially compensated by the induced charges in the metal layer. Meanwhile, the other type of charge sheet remains and generates a net electrical field that penetrates into the organic photoactive layer. This additional electrical field is determined by

$$
F=\frac{P}{\varepsilon_{0} \varepsilon_{F E}} \frac{d}{L}
$$

where $P$ is the polarization charge density, $d$ is the thickness of the $\mathrm{P}(\mathrm{VDF}-\mathrm{TrFE})$ layer, $L$ is the thickness of the organic photoactive layer, and $\varepsilon_{F E}$ is the relative dielectric constant of P(VDF-TrFE) (Yuan et al., 2011). The additional electric field caused by $\mathrm{P}(\mathrm{VDF}-\mathrm{TrFE})$ layer is also determined by the morphology of the ferroelectric layer. Generally, the P(VDF-TrFE) on P3HT:PCBM surface form nanomesa domains with a thin ferroelectric film surrounding it. Conductive AFM measurements indicate that the nanomesa domains play a major role in inducing the large electric field and the thin ferroelectric film surrounding the nanomesa is more efficient in charge collection. A number of studies have shown improved photovoltaic performance when a thin layer of ferroelectric layer with oriented dipoles is situated at one of the charge extraction contacts of the solar cell (Liu et al., 2013,Rastogi, 2013,Shin et al., 2014,Lazarev et al., 2015,Mai et al., 2015). The modification of surface charge can be established by capacitance spectroscopy (Hsiao et al., 2014).

(Asadi et al., 2011) suggested that that the improved efficiency is due to improvement of the cathode. A control of surface dipoles influences the charge injection properties of the contact by modifying the molecular band offsets (Wong et al., 2014). 
The direct rendering of dipole alignment within the ferroelectric blend layer is found to increase the carrier selectivity of the charge collecting interfaces up to two orders of magnitude (Jo et al., 2015) with a significant impact on $J-V$ curve performance as shown in Fig. 32.
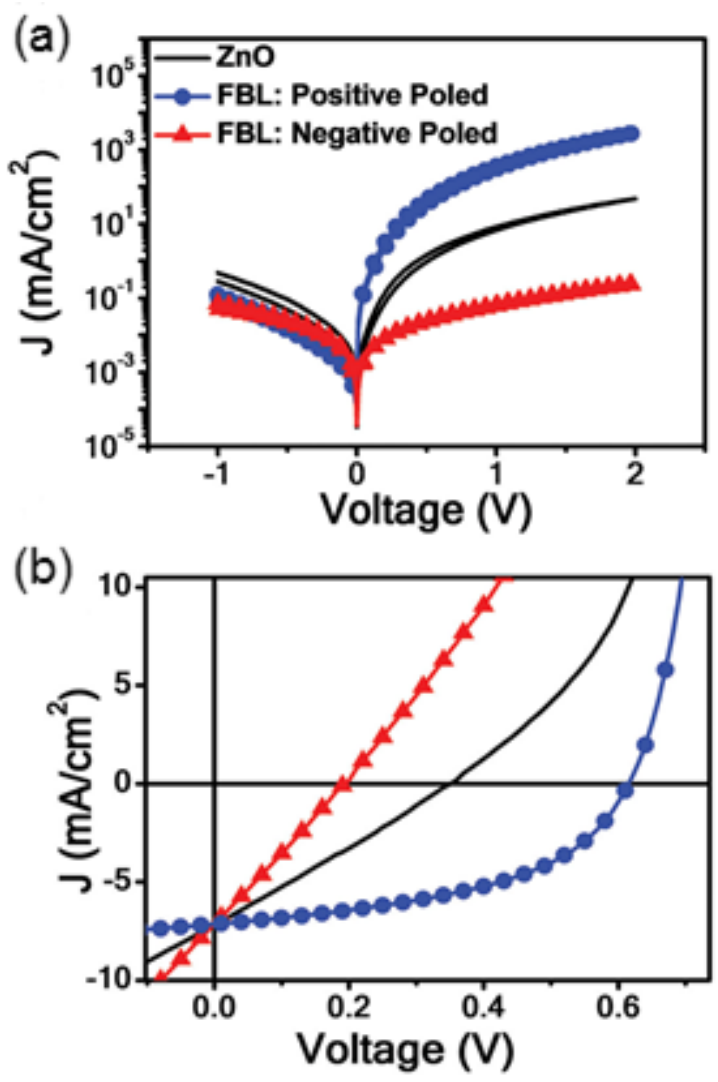

Fig. 32. (a) Electrical characteristics of a device ITO/ferroelectric blend layer(110 $\mathrm{nm}) / \mathrm{Al}$ under electrical poling. The blend layer is a mixture of $\mathrm{ZnO}$ and $\mathrm{P}(\mathrm{VDF}-\mathrm{TrFE})$. (b) Current density-voltage curves of bulk heterojunction organic solar cell, with a ferroelectric blend layer, at one sun illumination intensity. Reprinted from (Jo et al., 2015).

Another attractive application of using ferroelectric layers in bulk heterojunction organic solar cell devices is to reduce the energy loss caused by charge transfer between donor and acceptor, which leads to reduced open-circuit voltages. For efficient charge transfer, the offset between the LUMO of donor and the LUMO of acceptor should be larger than the binding energies in the donor materials. Generally, an energy level offset value of $0.3 \sim 0.5 \mathrm{eV}$ is sufficient. Offset values much larger than $0.3 \sim 0.5 \mathrm{eV}$ are improbable because of the reduced potential energy of the electron-hole pair after charge separation. For example, in the P3HT:PCBM system ( $\mathrm{Li}$ et al., 2005), the LUMO offset is as large as $1.0 \mathrm{eV}$, which makes the obtained $V_{\mathrm{OC}}(0.6 \mathrm{eV})$ much less than the optical bandgap of P3HT (2.0 eV). (Yang et al., 2012) demonstrated that inserting an ultra-thin 
layer of a ferroelectric material at the donor and acceptor interface is a straightforward method to tune the energy level offset between the donor and acceptor materials. The device structure and corresponding working principle of this method are shown in Fig. 33. The energy level shift $\Delta E_{L}$ caused by the aligned dipoles, can be described as

$$
\Delta E_{L}=\frac{q P}{\varepsilon_{0} \varepsilon_{F E}} d
$$

For P(VDF-TrFE) with a polarization charge density of $10 \mu \mathrm{C} / \mathrm{cm}^{2}$, a $0.6-\mathrm{nm}$-thick $\mathrm{P}(\mathrm{VDF}-\mathrm{TrFE})$ film can give an energy level shift of $0.8 \mathrm{eV}$, which should be sufficient to optimize the energy level offset in most of the donor/acceptor materials systems. As shown in Figure 33c, after aligning the dipoles of the ferroelectric layer with a negative external bias, the obtained $V_{\mathrm{OC}}$ increased from $0.55 \mathrm{~V}$ to $0.67 \mathrm{~V}$, which is higher than any other observed $V_{\mathrm{OC}}$ value based on the P3HT:PCBM system (Gevaerts et al., 2011,Lee et al., 2011). Here, the $V_{\text {OC }}$ improvement was also limited by the low coverage of the P(VDF-TrFE) on the P3HT surface $(\sim 20 \%)$.

(a)

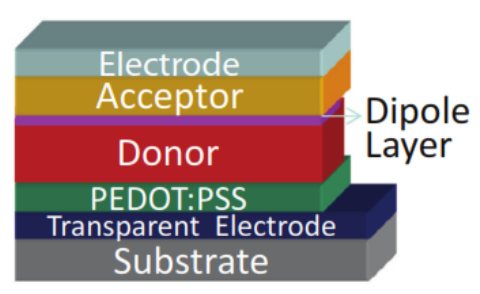

(b)

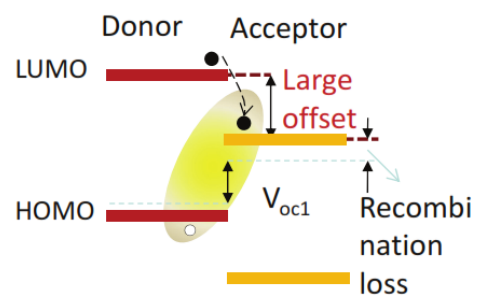

(c)

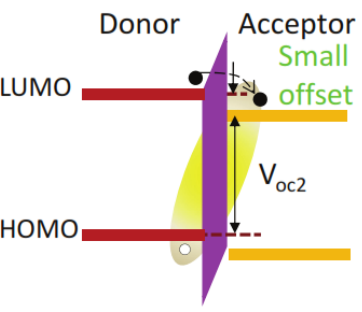

Fig. 33. (a) Structure of OPV devices with ferroelectric P(VDF-TrFE) interfacial layer inserted at D/A interface. (b) Energy diagram of donor-acceptor heterojunctions without ferroelectric layer, where the LUMO offset is large. (c) Energy diagram of donor-acceptor heterojunctions with ferroelectric layer at D/A interface, where the LUMO offset is reduced due to the aligned interfacial dipoles in the ferroelectric layer. Reproduced from (Yang et al., 2012).

Mixing the ferroelectric materials with the photoactive materials provides another approach to make use of the large local electrical field generated by ferroelectric molecules for improved exciton dissociation. Based on the classic dipole-electric field model, it was proposed by (Nalwa et al., 2012) that the local internal electrical field $\left(F_{\text {local }}\right.$ ) caused by the ferroelectric material dispersed in organic photoactive layer can be described as

$$
F_{\text {local }}=\frac{4 \pi \sigma_{P}}{\varepsilon_{0} \varepsilon_{F E}} f
$$

where $\sigma_{p}$ is the polarization charge density and $f$ is the volume fraction occupied by the dipoles. It was estimated that a small fraction $f$ of 0.03 can give a local electrical field of $\sim 240 \mathrm{~V} / \mu \mathrm{m}$, which should be sufficient for the dissociation of photogenerated e-h pairs 
$(\sim 50 \mathrm{~V} / \mu \mathrm{m})$. In the study of (Nalwa et al., 2012), the internal quantum efficiency of the OPV devices increased from about $70 \%$ to a very high value approaching $100 \%$ when $10 \%$ of $\mathrm{P}(\mathrm{VDF}-\mathrm{TrFE})$ was mixed into the P3HT:PCBM photoactive layer, which was explained as the improved exciton dissociation. This improved exciton dissociation rate caused by the large local electrical field was supported by a reduced photoluminescence lifetime in the photoactive layer with mixed $\mathrm{P}(\mathrm{VDF}-\mathrm{TrFE})(73 \mathrm{ps})$ as compared to that of the photoactive layer without the ferroelectric polymer (100 ps).

The strong modification of material surface by polarization charge prompted the interest to investigate the effects of ferroelectricity on the absorptive and catalytic properties of surfaces (Inoue et al., 1984, Yun and Altman, 2007,Zhang et al., 2011). The switchable polarization effects have been explored to regulate the surface chemistry (Kakekhani and Ismail-Beigi, 2015). (Li et al., 2012) prepared microcrystalline ferroelectric $\mathrm{PbTiO}_{3}$ cores coated with nanostructured $\mathrm{TiO}_{2}$ shells for photocatalysis applications. These developments have been reviewed by (Khan et al., 2016). The Solar energy production and storage by photoelectrochemical water splitting has recently fostered many research activities (Gimenez and Bisquert, 2016). The application of a ferroelectric layer has been adopted as a tool to enhance the rate of charge transfer at the semiconductor/electrolyte interface in reactions such as water splitting for photoelectrochemical fuel production. (Cao et al., 2014) and (Yang et al., 2015) demonstrated the control of charge transfer current by the state of polarization, as shown in Fig. 34.
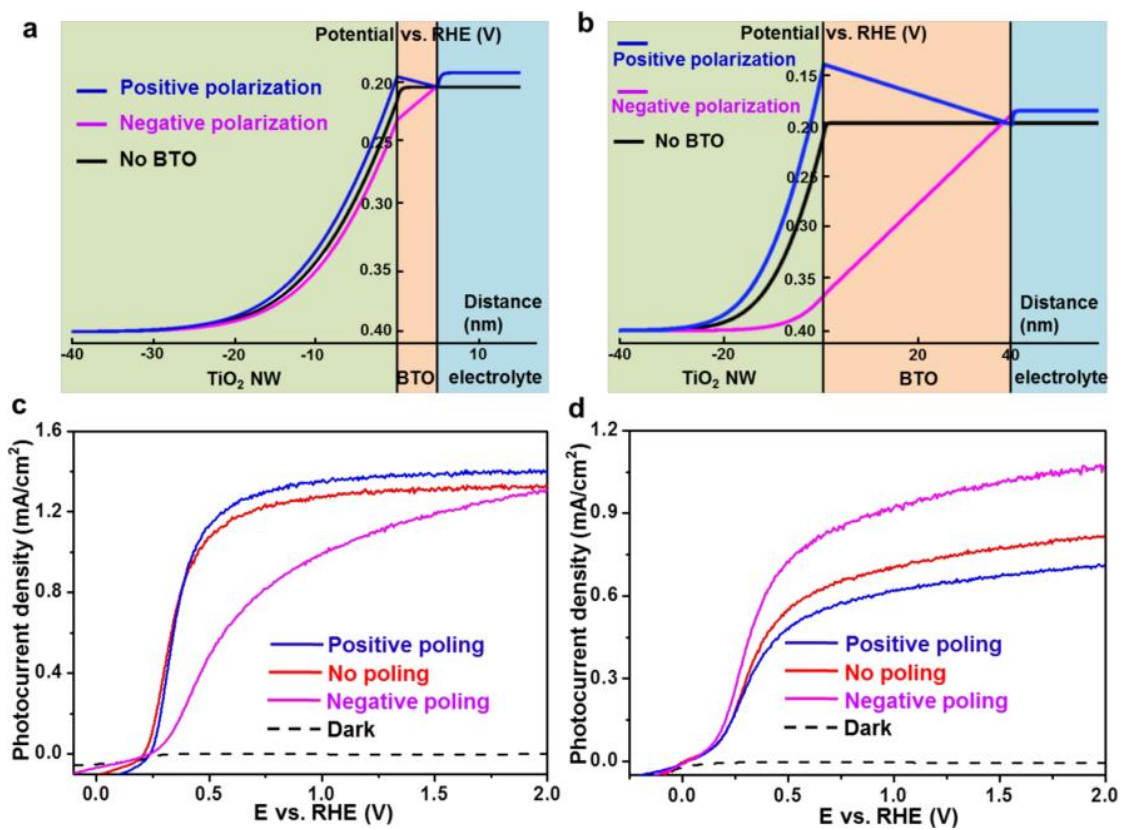

Fig. 34. (a) Calculated potential distribution and electric poling test of the ferroelectric enhancement. (a) Potential distribution of $150{ }^{\circ} \mathrm{C} \mathrm{TiO}_{2} / 5 \mathrm{~nm} \mathrm{BTO} / \mathrm{NaOH}$ heterojunction, where the spontaneous polarization in BTO shell induced an upward band bending of $\mathrm{TiO}_{2}$ core and facilitated the charge separation and transportation inside $\mathrm{TiO}_{2}$. (b) Potential distribution of the $210{ }^{\circ} \mathrm{C} \mathrm{TiO}_{2} / 40 \mathrm{~nm}$ BTO/NaOH heterojunctions, 
showing a strong electric field inside BTO, which facilitates the hole transport toward solution. (c) $\mathrm{J}-\mathrm{V}$ curves of the as-prepared (red), positively poled (blue) and negatively poled (magenta) $150{ }^{\circ} \mathrm{C} \mathrm{TiO}_{2} / \mathrm{BTO}$ NWs. (d) $\mathrm{J}-\mathrm{V}$ curves of the as-prepared (red), positively poled (blue) and negatively poled (magenta) $210{ }^{\circ} \mathrm{C} \quad \mathrm{TiO}_{2} / \mathrm{BTO}$ NWs. Reprinted from (Yang et al., 2015).

\section{Advanced materials concepts in ferroic photovolatics \\ 7.1. Ferroelectric oxide perovskites}

Here we briefly describe the main properties and potential applications of three of the most studied ferroelectric perovskite oxides: $\mathrm{LiNbO}_{3}, \mathrm{BaTiO}_{3}$ and $\mathrm{Pb}(\mathrm{Zr}, \mathrm{Ti}) \mathrm{O}_{3}$ and attractive strategies for further improvement of their power conversion efficiencies. Materials properties and preparation methods of a number of oxides materials have been recently reviewed by (Sheu et al., 2012).

$\mathrm{LiNbO}_{3}$ (LNO) is a ferroelectric material with a high Curie temperature of $\sim 1210{ }^{\circ} \mathrm{C}$ and a large spontaneous polarization of $\sim 70 \mu \mathrm{C} / \mathrm{cm}^{2}$. It exhibits piezoelectric, pyroelectric, electro-optic, photoelastic, large birefringent and bulk photovoltaic effect and is therefore being a promising material for a wide variety of optical applications in optoelectronics (Weis and Gaylord, 1985). The band gap of LNO single crystal is close to $4 \mathrm{eV}$, making it an attractive material for UV photodetector (Guo et al., 2009). The first studies on the bulk photovoltaic effect were performed on iron doped-LNO bulk. It was observed that when the material was exposed to uniform illumination under shortcircuit conditions, the photocurrent along the polar axis decayed. However, in opencircuit saturation conditions, atypically high voltages of $\sim 1000 \mathrm{~V}$ corresponding to fields of about $10^{5} \mathrm{~V} / \mathrm{cm}$ were observed, suggesting that it could not be generated by the same mechanisms as in conventional semiconductors. This effect accounted for the lightinduced changes in the refractive index, i.e. the photorefractive effect (Glass et al., 1974). Recently, Fe-doped LNO thin films have been reconsidered for solar batteries and it is shown that the effect of strain in epitaxial films along the polarization direction is an effective strategy to enhance the photovoltaic current (over 500 times) (Inoue et al., 2015).

$\mathrm{BaTiO}_{3}$ (BTO) is the first known ferroelectric, mostly in ceramic form, that undergoes a transition from the ferroelectric to the paraelectric phase upon heating above $130^{\circ} \mathrm{C}$, exhibiting an electronic bandgap of $3.4 \mathrm{eV}$ (Cardona, 1965). It has comprehensive applications including detectors, non-volatile memories, electro-optic devices and non-volatile dynamic access memory. The photovoltage of bulk BTO was shown to depend on the chemical composition and the grain size (Käppler and Arlt, 1981). An early report revealed that high photovoltage in polycrystalline BTO resulted from the integration of small photovoltages across spontaneously polarized individual BTO grains. Saturation field of $360 \mathrm{~V} / \mathrm{cm}$ and short-circuit photocurrents of 0.020 $\mu \mathrm{A} / \mathrm{cm}^{2}$ were reported for $\mathrm{CaTiO}_{3}$-doped BTO at room temperature and photovoltages of $0.55 \mathrm{~V}$ were identified across a single crystal (Brody, 1975). The shift current is yet 
another relevant mechanism in bulk BTO as photocurrent depends on both the magnitude of material polarization and on the electronic states with delocalized, covalent bonding along the current direction (Young and Rappe, 2012). As mentioned earlier (Zenkevich et al., 2014) have recently reported on the abnormal photovoltaic effect in heteroepitaxial ultrathin $\mathrm{BaTiO}_{3}$ films $(20-50 \mathrm{~nm})$ with a thickness similar or lower than the characteristic shift in the non-thermalized carriers $\left(l_{0}\right)$. They observed that the photoinduced electric field generated in the film and the energy conversion efficiency are four orders of magnitude higher $\left(\eta \sim 1 \%\right.$ and $\left.F_{\mathrm{pv}} \sim 3 \times 10^{5} \mathrm{~V} / \mathrm{cm}\right)$ than the bulk material. In this situation, all excited carriers are non-thermalized and contribute to the photovoltaic current (Sturman and Fridkin, 1992).

$\mathrm{PbZr}_{1-\mathrm{x}} \mathrm{Ti}_{\mathrm{x}} \mathrm{O}_{3}$ (PZT) thin films have attracted huge interest especially for their applications in electronic devices such as non-volatile memories, infrared sensors, optical shutters, modulators, actuators (Scott et al., 1988). PZT presents a distorted ferroelectric perovskite structure below $350^{\circ} \mathrm{C}$ (Cross, 2004). The gap on epitaxial films was 3.9 to $4.4 \mathrm{eV}$, depending on composition (Pintilie et al., 2007). Importantly, their properties are strongly dependent on the cation Zr:Ti ratio and crystal orientation. Highspeed ( 10 ns) non-destructive polarization-dependent photoresponse was evaluated in PZT to be further used as non-destructive read out (NDRO) ferroelectric memories (Thakoor, 1993). The origin of the photoresponse at low electric fields or in remnent polarization was identified as the bulk photovoltaic effect (Kholkin et al., 1998). (Pintilie et al., 1998) studied the influence of the metal-ferroelectric interface in PZT films on $\mathrm{SrRuO}_{3}$ with different top metal electrodes ( $\mathrm{Au}, \mathrm{Ag}, \mathrm{Pt}, \mathrm{Cu}, \mathrm{Ni}, \mathrm{Cr}$ ). This study allowed them to conclude that the photovoltaic effect observed in PZT epitaxial films was different from the photovoltaic effect observed in bulk ferroelectrics although the latter cannot be excluded. If present, the bulk contribution could be related to the presence of imprint fields as further confirmed by piezo-response scanning force microscopy (Alexe et al., 2001).

To improve the photovoltaic effect in PZT, several strategies have been adopted. For example, the incorporation of a $\mathrm{Cu}_{2} \mathrm{O}$ (n-type) layer between the PZT and the Pt cathode contact has been demonstrated to be very effective to enhance the short-circuit photocurrent 120-fold (up to $4.8 \mathrm{~mA} / \mathrm{cm}^{2}$ ) and the power conversion efficiency under AM1.G illumination 72-fold ( $\mathrm{CaO}$ et al., 2012). The role of $\mathrm{Cu}_{2} \mathrm{O}$ is to improve the energy level alignment to extract electrons from $\mathrm{PZT}$ to $\mathrm{Cu}_{2} \mathrm{O}$, see Fig. 35 .

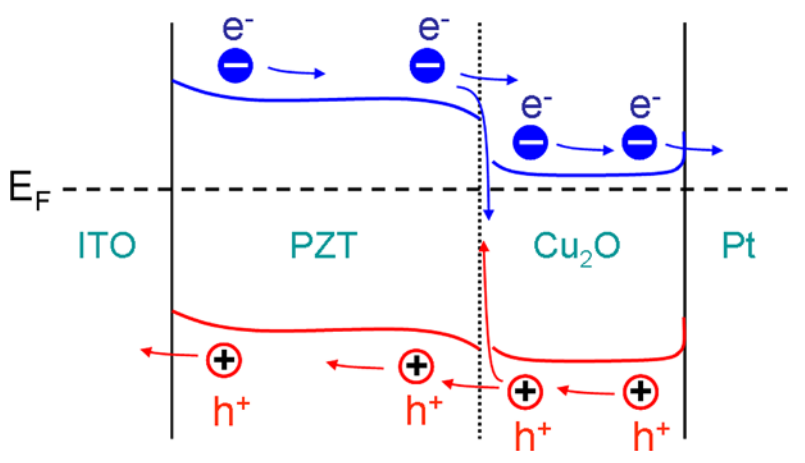


Fig. 35. Band diagram for ITO/PZT/Cu $\mathrm{Cu}_{2} \mathrm{O}$ Pt. Reprinted from (Cao et al., 2012).

Alternatively, in order to minimize the built-in fields due to Schottky barriers and favor the polarization induced electric field, a micron-thick La-doped PZT has been used to test different metal electrodes. Therefore, by tuning the photoelectric effect the PV effect can be enhanced. Best results are obtained using $\mathrm{Mg}$ with open circuit voltage and short circuit current density of $8.34 \mathrm{~V}$ and $3.25 \mathrm{nA} / \mathrm{cm}^{2}$, respectively, under AM1.5 illumination (Zhang et al., 2013).

Studies to design and engineering ferroelectric oxides with low band gap without jeopardizing ferroelectricity is at the core of current research. From first principles calculations it has been proposed several strategies ranging from polarization rotation to the incorporation of intermediate bands (Wang et al., 2015). The characteristics of the transition metal-oxygen chemical bond has a strong effect on the electronic properties of perovskite oxides giving rise to a broad assortment of band gaps within the ferroelectric perovskite family ranging from $5.7 \mathrm{eV}$ for $\mathrm{LaScO}_{3}$ to $2 \mathrm{eV}$ for $\mathrm{WO}_{3}$. In addition, for the same composition, rhombohedral phase gives larger band gap than the tetragonal phase . (Wang et al., 2014). Also, several polar oxides with the $\mathrm{LiNbO}_{3}$ structure have also emerged as potential candidates with strong bulk photovoltaic effect and low bandgaps. Compositions with $\mathrm{Pb}^{4+}$ and $\mathrm{Bi}^{5+}$ ensures the absence of $d$-states at the band edge and the conduction bands are formed by $s$-states hybridized with oxygen $p$-states (Young et al., 2015). Finally, nanolayered architectures have also been proposed as an emerging approach to tuning the absorption properties and bulk photovoltaic effect where an increase by a factor of 43 has been predicted in Ni-doped $\mathrm{PbTiO}_{3}$ system (Wang et al., 2016).

Recent experimental demonstration of visible-light absorption has been observed in layered ferroelectric bismuth titanate with $\mathrm{LaCoO}_{3}$ as alloying material where the bandgap has been reduced by $1 \mathrm{eV}$ (Choi et al., 2012) and ferroelectric perovskites $(\mathrm{K}, \mathrm{Ba})(\mathrm{Ni}, \mathrm{Nb}) \mathrm{O}_{3-\delta}$ solid solutions achieving a bandgap variation from 1.1 to $3.8 \mathrm{eV}$ (Grinberg et al., 2013). Therefore, these approaches should encourage further exploration of ferroelectric perovskite oxide materials (and also multiferroic materials described in the next section) for solar energy applications.

\subsection{Multiferroic materials}

Multiferroic materials are ferroelectric systems which also exhibit a magnetic order parameter. During the last decade or so, a number of such systems were successfully synthesized, with the aim of exploiting their multiple functionalities for device applications. Notable examples include $\mathrm{BiFeO}_{3}(\mathrm{BFO}), \mathrm{BiMnO}_{3}(\mathrm{BMO})$ and $\mathrm{Bi}_{2} \mathrm{FeCrO}_{6}$ (BFCO) (Nechache et al., 2006,Nechache et al., 2007). An interesting property of these materials is that they have a significantly reduced band gap with respect to conventional 
ferroelectrics, due to the pronounced electron-electron coupling. These materials therefore combine an effective separation of charges in the bulk with a small semiconductor bandgap. BFO is an example of an extensively studied multiferroic material, which is ferroelectric and antiferromagnetic; it exhibits a band gap of about 2.6-2.7 eV (Basu et al., 2008). In addition, a large photovoltage of about $15 \mathrm{~V}$ in epitaxial BFO thin films was recently reported (Yang et al., 2010). Following the theoretical predictions of (Baettig and Spaldin, 2005), thin films of the double perovskite BFCO were successfully synthesized by pulsed laser deposition (PLD). Epitaxial BFCO thin films were shown to have strong internal fields with a saturated polarization of about $95 \mu \mathrm{C} / \mathrm{cm}^{2}$ along the [111] pseudocubic direction (Nechache et al., 2009). BFCO is also a ferrimagnetic double perovskite with magnetic moment that depends on the degree of $\mathrm{Fe} / \mathrm{Cr}$ cation ordering in the films (Ichikawa et al., 2008). Optical to electric PCEs up to $6.5 \%$ were initially demonstrated under light wavelength $\lambda$ of $635 \mathrm{~nm}$ in epitaxial BFCO thin films grown by PLD (Nechache et al., 2011). These films showed a high short-circuit current density $\left(J_{\mathrm{sc}}\right)$ of $0.99 \mathrm{~mA} / \mathrm{cm}^{2}$, this magnitude being related to $\mathrm{Fe}-\mathrm{Cr}$ cationic ordering. The effect of cationic ordering on the PV properties was even more evident in $\mathrm{BFO} / \mathrm{BiCrO}_{3}(\mathrm{BCO})$ heterostructures (Chakrabartty et al., 2014), where photocurrent density and photovoltage can be tuned by varying the thickness and number of respective $\mathrm{BFO} / \mathrm{BCO}$ bilayers (i.e. $\mathrm{Fe}-\mathrm{O}-\mathrm{Cr}$ interfaces). On the other hand, BMO thin films exhibit the lowest bandgap, namely $1.1 \mathrm{eV}$, thanks to charge transfer excitations between mixed $\mathrm{O} 2 p$ and $\mathrm{Mn} 3 d$ states and have ferroelectric polarization of $25 \mu \mathrm{C} / \mathrm{cm}^{2}$. External solar power conversion efficiencies up to $0.1 \%$ in BMO thin films grown onto (111) oriented Niobium doped $\mathrm{SrTiO}_{3}$ substrates were recently reported (Chakrabartty et al., 2014). The results showed that the photocurrent and photovoltage strongly depend on the $\mathrm{Bi} / \mathrm{Mn}$ ratio in the film, with the best power conversion efficiency obtained for films with high $\mathrm{Bi} / \mathrm{Mn}$ ratio and thus a high $\mathrm{BMO}$ phase content.

The search for new interesting multiferroic materials for photovoltaics has led to the preparation of $\mathrm{KBiFe}_{2} \mathrm{O}_{5}$, a polar and layered compound made of transition metal $\mathrm{MO}_{4}$ tetrahedral network structures. Single crystal samples show high $\mathrm{T}_{\mathrm{c}}(780 \mathrm{~K})$ and small optical bandgap (1.6 eV) achieving high photovoltages, $8.8 \mathrm{~V}$, and photocurrents (15 $\mu \mathrm{A} / \mathrm{cm}^{2}$ ) (Zhang et al., 2013,Zhai et al., 2015). Such small bandgap has been attributed to the covalent character of $\mathrm{Fe}-\mathrm{O}$ bonds in $\mathrm{FeO}_{4}$ tetrahedra that alternates with $\left[(\mathrm{K}, \mathrm{Bi}) \mathrm{O}_{2}\right]$ blocks, along with the $\mathrm{t}_{2 \mathrm{~g}} / \mathrm{e}_{\mathrm{g}}$ orbitals inversion. Although being weakly ferroelectric, these findings open a new avenue to discover new absorber layers based on the brownmillerite $\left(\mathrm{A}_{2} \mathrm{~B}_{2} \mathrm{O}_{5}\right)$ structure.

A breakthrough in the field of ferroelectric photovoltaics (FePV) was reported in a recent study on BFCO, which exhibits both a small bandgap and a large photocurrent density $\left(11.7 \mathrm{~mA} \mathrm{~cm}^{-2}\right.$ ), yielding a PCE as high as $3.3 \%$ in single layer films (Nechache et al., 2015). By controlling the Fe/Cr cationic order and their distribution in the films, it was reported that the band gap of BFCO can be tuned in the range 2.1 to $1.4 \mathrm{eV}$. This 
led to the design of a multi-absorber system based on BFCO, obtained by stacking three layers with different bandgaps, leading to a breakthrough PCE of 8.1\%, see Fig. 17.

These investigations suggest that adequate combinations between optical and ferroelectric properties are critical to achieve high efficiency multiferroic PV devices. In such materials, low band gap and large ferroelectric polarization values promote an effective charge carrier generation and separation. Under certain conditions, BFCO with higher $\mathrm{Fe} / \mathrm{Cr}$ cation ordering showed a very low band gap (1.2-1.4 eV) accompanied with weak ferroelectricity, see Figs. 36 (a) and (b). Despite a larger absorption of sunlight by semiconducting BFCO films, the weak ferroelectric polarization induced internal electric field decreases the separation power of photogenerated carriers and increases losses due to recombination.
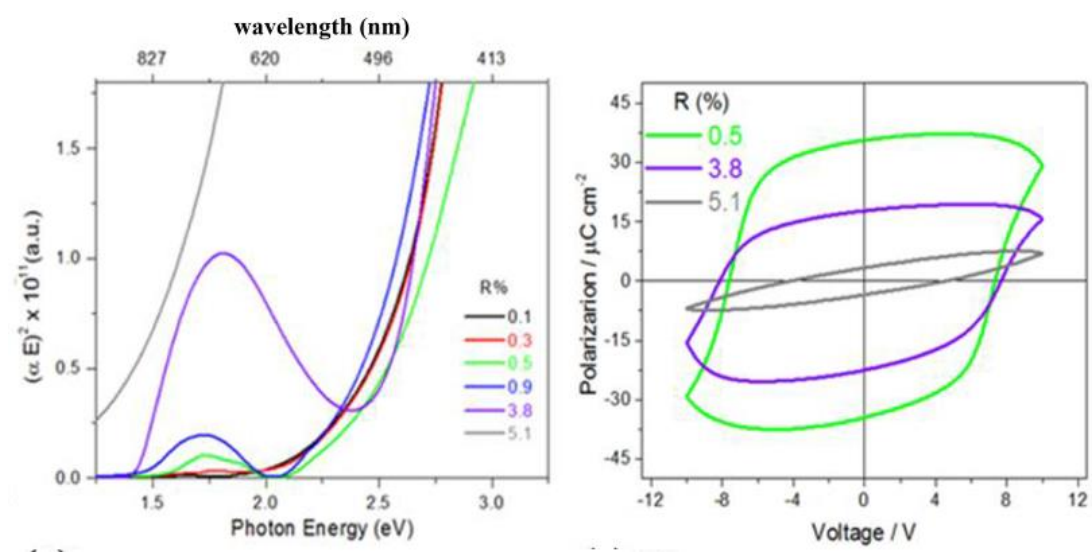

Fig. 36. (a) Direct band gap of BFCO thin films with various $\mathrm{Fe} / \mathrm{Cr}$ ordering ( $\mathrm{R} \%)$. (b) Polarization of BFCO thin films with various $\mathrm{R} \%$.

A promising future direction consists in forming a $\mathrm{p}-\mathrm{i}-\mathrm{n}$ heterojunction and multijunction solar cells where uniform thin-film processing and balanced electron and hole collection is achieved. The tunable optical and electrical properties of MFs can be simply modulated and optimized to implement them in PV cell architectures and promote the development of high efficiency and cost effective solar cells.

$p-i-n$ heterojunction. PV devices using semiconductor materials such as amorphous and microcrystalline silicon or germanium are made using the $p-i-n$ configuration. Here, an intrinsic semiconductor is sandwiched between heavily doped $p$ - and $n$-layers, as indicated in section 2. Semiconductor materials absorb a certain portion of the solar spectrum and adjacent layers (electrodes) are required to extract the photo-generated carriers to an external electrical load. Semiconducting BFCO has suitable optical and electrical properties and can be used as absorber in a $p-i-n$ heterostructure. To achieve high efficiency devices based on such materials, adjacent layers, namely electron and hole transporter materials (ETM and HTM) have to be chosen carefully in relation to their optical and electronic properties. In addition, since cationic ordering in BFCO is promoted only by epitaxial growth, these materials should have crystal properties closer 
to that of BFCO. This will ensure epitaxial growth of the overall heterostructure with high crystal, interface quality and low defect densities. The most promising candidates, including $\mathrm{NiO}_{\mathrm{x}}$ (Sato et al., 1993), $\mathrm{MoO}_{3}$ (Hamwi et al., 2009), indium doped $\mathrm{SrTiO}_{3}$ (Huang et al., 2016), and niobium doped $\mathrm{SrTiO}_{3}$ (Fukushima and Shibagaki, 1998), magnesium or aluminum doped $\mathrm{ZnO}$ (Karger and Schilling, 2005), as p- and n-type materials respectively are given in Fig. 37(a). In addition to the possibility of growing them epitaxially on perovskite materials, these oxides exhibit optical and electronic properties suitable for achieving high efficiency BFCO based $p-i-n$ heterojunction solar cells.

The device performance of BFCO solar cells will be highly dependent on their architectures, which will in turn impose the choice of materials, the deposition methods for the material and the compatibility between the different components in the device. Two major embodiments of BFCO solar cells may be developed, i.e. inverted or conventional planar architecture configurations, depending on the direction of the electrical current Fig. 37 (b) and (c). In such heterostructure, BFCO thin films can act as intrinsic absorber sandwiched between $p$ - and $n$-type semiconductor oxides as hole- and electron- collecting layer, respectively. The small bang gap of BFCO is expected to lead to PV devices with enhanced carrier collection efficiency.

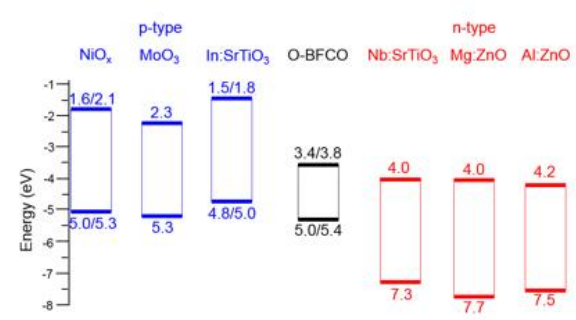

(a)

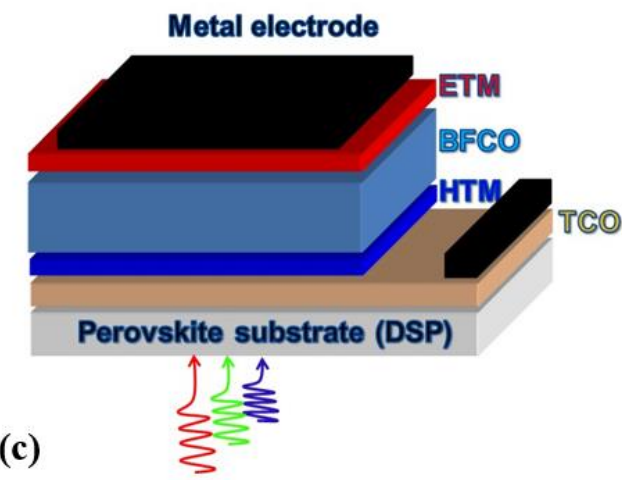

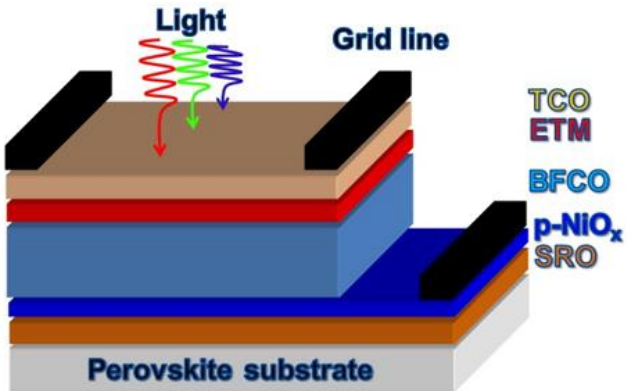

(b)

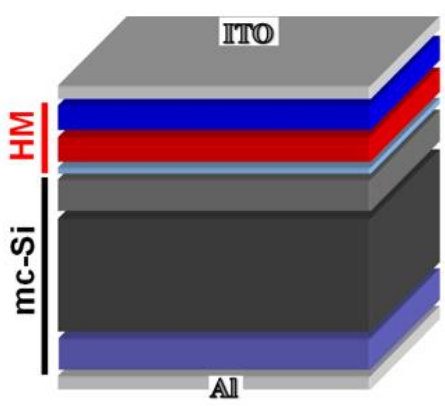

(d)

Fig. 37. (a) Schematic descriptions of the energy level diagram of $\left(\mathrm{NiO}, \mathrm{MoO}_{3}\right) \mathrm{In}-$ $\mathrm{STO} / \mathrm{BFCO} / \mathrm{Nb}-\mathrm{STO}$ (Al:ZnO, Mg:ZnO). Structure of (b) conventional and (c) inverted $p-i-n$ junction types of semiconducting BFCO PV devices. (d) Schematic representation 
of the mc-Si / HM tandem photovoltaic cell.

Multijunction Solar Cells. Another route to high efficiency PV devices consists in applying auxiliary layer to existing types of cells in order to enhance their PCE by forming tandem devices. The optical properties of BFCO thin films can be modulated and optimized to match those of crystalline silicon and other thin films used in devices such as n-CdS/p-CuInSe ${ }_{2}$ (CIS), n-CdS/p-CuIn ${ }_{1-x} \mathrm{Ga}_{\mathrm{x}} \mathrm{Se}_{2}$ (CIGS), and $\mathrm{Cu}_{2} \mathrm{ZnSnSe}_{4}$ (CZTSSe), when used as a top cell in a tandem junction (Beiley and McGehee, 2012,Nechache et al., 2012). Such materials could be implemented using a simple method such as sputtering or chemical vapor deposition, which allowed enhancing the PV conversion efficiency of single semiconductor solar cells by exploiting the multifunctional properties of low cost multiferroic oxides.

Due to Auger recombination, which mainly limits the photogenerated carriers lifetime (Green, 1984), a significant increase in efficiency for crystalline silicon cells is improbable. In addition, the development of wide-bandgap top cells for tandem junctions based on silicon and thin film PV cells still remains a challenge for future generation PV devices (Nechache et al., 2006,La et al., 2014). In fact, this device architecture requires a careful management of the absorbed light radiation, where shortwavelength light should be preferentially absorbed in the top cell and long-wavelength light must be transmitted unrestricted to the bottom cell. Thanks to the tunable optical and electrical properties of perovskite oxides, placing such a material on top of a silicon cell is expected to enhance the performance of existing PV technologies with simultaneous cost effectiveness (Basu et al., 2008).

The realization of this cell requires a material or a single multiferroic heterostructure, thus distinguishing it from the many complex structures (e.g., anti-reflection layer, grooves, buried contact and back surface field, etc..) that are currently available on the market. The film thicknesses of materials required for efficient light absorption can be reduced by at least one order of magnitude compared to currently available technology, thereby significantly reducing the cost of materials. In addition, the low optical gaps of MFs allow decreasing the likelihood of carrier recombination and lead to a significant increase in the photocurrent density, consequently increasing the PCE of the cell. These band gaps can be adjusted by changing the specific crystalline structure and chemical composition of the materials. The relative abundance of the required materials and their simple manufacturing processes may allow to achieve such substantial improvements at low cost. Bismuth, being a heavy metal, however, is considered non-toxic and this is also the case for the oxides of chromium and iron. The realization of devices based on this technology does not require installing specific sealing processes since MF oxides do not degrade in ambient atmosphere. This would help to stabilize cell performance over time and increase its current lifespan (10 years).

The promising potential of using perovskite $\mathrm{BFCO}$ as a complement to mature PV technologies like silicon has been recently demonstrated (Nechache et al, 2016). The 
semiconducting bandgap of the ordered BFCO phase combined with its ferroelectric properties, opens the possibility of a ferroelectric PV efficiency above $2 \%$ in BFCO thin films epitaxially gown on silicon through a buffer layer of $\mathrm{MgO}$. Due to the direct relationship between $\mathrm{Fe} / \mathrm{Cr}$ cation ordering and the $\mathrm{PV}$ effect in $\mathrm{BFCO}$ and since this ordering is only possible in epitaxial films, the epitaxial growth of highly ordered BFCO on silicon was a critical challenge to achieve high efficiency tandem junction device configurations. In addition, recent findings validated the use of BFCO as photocathode material and demonstrated that the manipulation of internal fields through polarization in ferroelectric materials is an alternative pathway for the design of improved photoelectrodes and smart devices for solar energy conversion (Li et al., 2015).

\section{Conclusion and outlook}

We have reviewed the physical aspects of ferroelectric semiconductors for PV solar energy conversion. We analyzed a variety of phenomena relevant to device operation such as the polarization-modulated Schottky-like barriers at metal-ferroelectric interfaces, depolarization fields, vacancy migration, above-bandgap photovoltage, and the switchable rectifying behavior of ferroelectric thin films. We have included ferroelectric effects in the physical model of a solar cell. Our work provides a general picture of the influence of ferroelectric effects on the actual PCE of the solar cell device. From numerical simulation, we concluded that symmetric Schottky MFM structures are necessary to obtain a noticeable switchable behavior or a large hysteresis loop in the $J-V$ curves under illumination. Both effects associated with the ferroelectric polarization surface polarization charges and spatial dependent polarization are necessary, to interpret the experimental $J-V$ curves obtained so far. This effect allows the switching performance in $J-V$ curves in symmetric Schottky solar cells. We have also discussed the bulk photovoltaic effect and how the energy barriers at ferroelectric-ferroelectric and metal-ferroelectric interfaces are modified by the ferroelectric effect. We have also examined the perovskite ferroelectric materials that have provided the best photovoltaic performance.

Ferroelectricity and photovoltaics are two disciplines that traditionally have been treated separately, although the coupling of light absorption with ferroelectricity has been known for years. The recent demonstration of ferroelectric oxide materials that exhibit visible light absorption along with high-efficiencies in emerging perovskite halide PV materials have radically boosted the field. There are three main approaches to build improved PV devices based on semiconductor ferroelectric materials. One direction is to increase the photocurrent, which can be obtained by bandgap engineering (manipulation of chemical composition and epitaxy including doping, gradient composition, strain, unit cell structure) as well as by improving geminate charge dissociation. Based on the metal-semiconductor-ferroelectric interface, the second method uses the enhancement of charge collection efficiency and the depolarization field. In this regard, phenomena such as: the formation of a depletion layer, electrochemical migration of defects and the associated change in electronic structure, 
the bulk photovoltaic effect itself, and the screening of the metal contact must be treated in the future. Yet another direction is represented by the combination of ferroelectric oxide perovskites with conventional (silicon) or emerging (organic, lead halide perovskite) PV technologies which could result in greater performance due to an improved light harvesting combined with a potentially higher generation yield afforded by the permanent dipole of the ferroelectric material. There is no theoretical estimate of the maximum efficiency of this type of cells, yet the lack of thermodynamic limits of abnormal PV effect could boost existing values, provided that new materials are found that take the bulk effect to high performance levels. In addition, ferroelectric perovskite oxides show effects that are not present in classical semiconductor based systems, such as switchable photo-currents or photostriction, stimulating huge interest for novel sensing devices with non-contact remote control or photo-ferroelectric memory. Therefore, ferroelectric semiconductors offer the possibility to develop new know-how and a plethora of new functionalities of man-made materials for sensing, large scale energy conversion, energy storage, data retention, autonomous energy harvesting at small scales. The field is still very young and we anticipate that new, rich physical insights will emerge as an ever growing community focuses on these fascinating phenomena.

\section{Acknowledgements}

The research leading to these results has received funding from the European Union Seventh Framework Program [FP7/2007-2013] under grant agreement 316494.

\section{References}

Akbashev, A. R.; Fridkin, V. M.; Spanier, J. E. in Nanoscale Ferroelectrics and Multiferroics; John Wiley \& Sons, Ltd, 2016; pp 830-850.

Alexe, M.; Harnagea, C.; Hesse, D.; Gösele, U. "Polarization imprint and size effects in mesoscopic ferroelectric structures". Applied Physics Letters 2001, 79, 242-244.

Anshul, A.; Kumar, A.; Gupta, B. K.; Kotnala, R. K.; Scott, J. F.; Katiyar, R. S. "Photoluminescence and time-resolved spectroscopy in multiferroic BiFeO3: Effects of electric fields and sample aging". Applied Physics Letters 2013, 102, 222901.

Asadi, K.; de Bruyn, P.; Blom, P. W. M.; de Leeuw, D. M. "Origin of the efficiency enhancement in ferroelectric functionalized organic solar cells". Applied Physics Letters 2011, 98, 183301.

Baettig, P.; Ederer, C.; Spaldin, N. A. "First principles study of the multiferroics $\mathrm{BiFeO} 3, \mathrm{Bi} 2 \mathrm{FeCrO} 6$, and $\mathrm{BiCrO} 3$ : Structure, polarization, and magnetic ordering temperature". Physical Review B 2005, 72.

Baettig, P.; Spaldin, N. A. "Ab initio prediction of a multiferroic with large polarization and magnetization". Applied Physics Letters 2005, 86, 012505. 
Basu, S. R.; Martin, L. W.; Y.-H. Chu; Gajek, M.; Ramesh, R.; Rai, R. C.; Xu, X.; Musfeldt, J. L. "Photoconductivity in $\mathrm{BiFeO}_{3}$ thin films". Applied Physics Letters 2008, 92, 091905.

Batra, I. P.; Wurfel, P.; Silverman, B. D. "Phase Transition, Stability, and Depolarization Field in Ferroelectric Thin Films". Physical Review B 1973, 8, 3257 3265 .

Beiley, Z. M.; McGehee, M. D. "Modeling low cost hybrid tandem photovoltaics with the potential for efficiencies exceeding 20\%". Energy \& Environmental Science 2012, 5, 9173-9179.

Belinicher, V. I. Sturman, B. I. "Photogalvanic Effect in Media Without Symmetry Centers". Uspekhi Fizicheskikh Nauk 1980, 130, 415-458.

Bhatnagar, A.; Roy Chaudhuri, A.; Heon Kim, Y.; Hesse, D.; Alexe, M. "Role of domain walls in the abnormal photovoltaic effect in BiFeO3". Nat Commun 2013, 4.

Bisquert, J. Nanostructured Energy Devices: Equilibrium Concepts and Kinetics; CRC Press: Boca Raton, 2014.

Bisquert, J.; Cahen, D.; Rühle, S.; Hodes, G.; Zaban, A. "Physical chemical principles of photovoltaic conversion with nanoparticulate, mesoporous dye-sensitized solar cells.". The Journal of Physical Chemistry B 2004, 108, 8106-8118.

Bisquert, J.; Garcia-Belmonte, G. "On Voltage, Photovoltage, and Photocurrent in Bulk Heterojunction Organic Solar Cells". Journal of Physical Chemistry Letters 2011, 2, 1950-1964.

Blom, P. W. M.; Wolf, R. M.; Cillessen, J. F. M.; Krijn, M. P. C. M. "Ferroelectric Schottky Diode". Physical Review Letters 1994, 73, 2107-2110.

Brody, P.; Crowne, F. "Mechanism for the high voltage photovoltaic effect in ceramic ferroelectrics". JEM 1975, 4, 955-971.

Brody, P. S. "Large polarization-dependent photovoltages in ceramic BaTiO3 + 5 wt.\% CaTiO3". Solid State Communications 1973, 12, 673-676.

Brody, P. S. "High voltage photovoltaic effect in barium titanate and lead titanatelead zirconate ceramics". Journal of Solid State Chemistry 1975, 12, 193-200.

Bube, R. H. Photoelectronic Properties of Semiconductors; Cambridge U. P.: Cambridge, 1992.

Butler, K. T.; Frost, J. M.; Walsh, A. "Ferroelectric materials for solar energy conversion: photoferroics revisited". Energy \& Environmental Science 2015, 8, 838848.

Cao, D.; Wang, Z.; Nasori; Wen, L.; Mi, Y.; Lei, Y. "Switchable Charge-Transfer in the Photoelectrochemical Energy-Conversion Process of Ferroelectric $\mathrm{BiFeO} 3$ Photoelectrodes". Angewandte Chemie 2014, 126, 11207-11211. 
Cao, D.; Zhang, H.; Fang, L.; Dong, W.; Zheng, F.; Shen, M. "Polarization effect on the photocurrent of Pt sandwiched multi-crystalline ferroelectric films". Materials Chemistry and Physics 2011, 129, 783-786.

Cao, D. W.; Wang, C. Y.; Zheng, F. G.; Dong, W.; Fang, L.; Shen, M. R. "HighEfficiency Ferroelectric-Film Solar Cells with an n-type Cu2O Cathode Buffer Layer". Nano Letters 2012, 12, 2803-2809.

Cao, Y.; Shen, J.; Randall, C. A.; Chen, L. Q. "Phase-field modeling of switchable diode-like current-voltage characteristics in ferroelectric BaTiO3". Applied Physics Letters 2014, 104, 182905.

Cardona, M. "Optical Properties and Band Structure of SrTO3 and BaTiO3". Physical Review 1965, 140, A651-A655.

Chakrabartty, J.; Nechache, R.; Nicklaus, M.; Li, S.; Ruediger, A.; Rosei, F. "Photovoltaic effect in multiphase Bi-Mn-O thin films". Optics Express 2014, 22, A80 A89.

Chakrabartty, J.; Nechache, R.; Nicklaus, M.; Li, S.; Ruediger, A.; Rosei, F. "Photovoltaic Properties of Multiferroic $\mathrm{BiFeO}_{3} / \mathrm{BiCrO}_{3}$ Heterostructures". Journal of American Ceramic Society 2014, 97, 1837-1840.

Chandra, P. L., P. B. A Landau Primer for Ferroelectrics. In eprint arXiv:condmat/0609347, 2006; Vol. 105; pp 69-116

Choi, W. S.; Chisholm, M. F.; Singh, D. J.; Choi, T.; Jellison, G. E.; Lee, H. N. "Wide bandgap tunability in complex transition metal oxides by site-specific substitution". Nature Communications 2012, 3.

Clark, S. J.; Robertson, J. "Band gap and Schottky barrier heights of multiferroic BiFeO3". Applied Physics Letters 2007, 90, 132903.

Coll, M.; Gomez, A.; Mas-Marza, E.; Almora, O.; Garcia-Belmonte, G.; CampoyQuiles, M.; Bisquert, J. "Polarization Switching and Light-Enhanced Piezoelectricity in Lead Halide Perovskites". The Journal of Physical Chemistry Letters 2015, 1408-1413.

Cross, E. "Materials science: Lead-free at last". Nature 2004, 432, 24-25.

Fan, Z.; Sun, K.; Wang, J. "Perovskites for photovoltaics: a combined review of organic-inorganic halide perovskites and ferroelectric oxide perovskites". Journal of Materials Chemistry A 2015, 3, 18809-18828.

Fan, Z.; Xiao, J.; Sun, K.; Chen, L.; Hu, Y.; Ouyang, J.; Ong, K. P.; Zeng, K.; Wang, J. "Ferroelectricity of $\mathrm{CH} 3 \mathrm{NH} 3 \mathrm{PbI} 3$ Perovskite". The Journal of Physical Chemistry Letters 2015, 6, 1155-1161.

Fang, L.; You, L.; Zhou, Y.; Ren, P.; Shiuh Lim, Z.; Wang, J. "Switchable photovoltaic response from polarization modulated interfaces in $\mathrm{BiFeO} 3$ thin films". Applied Physics Letters 2014, 104, 142903. 
Filippetti, A.; Delugas, P.; Saba, M. I.; Mattoni, A. "Entropy-Suppressed Ferroelectricity in Hybrid Lead-Iodide Perovskites". The Journal of Physical Chemistry Letters 2015, 6, 4909-4915.

Fridkin, V. M. Photoferroelectrics; Springer: Berlin, 1979.

Fukushima, K.; Shibagaki, S. "Nb doped $\mathrm{SrTiO}_{3}$ thin films deposited by pulsed laser ablation". Thin Solid Films 1998, 315, 238-243.

Gärtner, W. "Depletion-Layer Photoeffects in Semiconductors". Physical Review 1959, 116, 84-87.

Ge, C.; Jin, K.-J.; Wang, C.; Lu, H.-B.; Wang, C.; Yang, G.-Z. "Numerical investigation into the switchable diode effect in metal-ferroelectric-metal structures". Applied Physics Letters 2011, 99, 063509.

Gevaerts, V. S.; Koster, L. J. A.; Wienk, M. M.; Janssen, R. A. "Discriminating between bilayer and bulk heterojunction polymer: fullerene solar cells using the external quantum efficiency". ACS applied materials \& interfaces 2011, 3, 3252-3255.

Gimenez, S.; Bisquert, J. Photoelectrochemical Solar Fuel Production. From Basic Principles to Advanced Devices; Springer, 2016.

Glass, A. M.; von der Linde, D.; Negran, T. J. "High-voltage bulk photovoltaic effect and the photorefractive process in LiNbO3". Applied Physics Letters 1974, 25, 233-235.

Goldstein, B.; Pensak, L. "High-Voltage Photovoltaic Effect". Journal of Applied Physics 1959, 30, 155-161.

Green, M. A. "Limits on the Open-circuit Voltage and Efficiency of Silicon Solar Cells Imposed by Intrinsic Auger Processes". IEEE Trans. Electron. Devices 1984, 5, 671-678.

Grekov, A. A.; Malitskaya, M. A.; Spitsina, V. D.; Fridkin, V. M. "Photoelectric effects in A5B6C7-type ferroelectrics-semiconductors with low-temperature phase transitions". Kristallografiya 1970, 15, 500-509.

Grinberg, I.; West, D. V.; Torres, M.; Gou, G. Y.; Stein, D. M.; Wu, L. Y.; Chen, G. N.; Gallo, E. M.; Akbashev, A. R.; Davies, P. K.; Spanier, J. E.; Rappe, A. M. "Perovskite oxides for visible-light-absorbing ferroelectric and photovoltaic materials". Nature 2013, 503, 509-+.

Guerrero, A.; Garcia-Belmonte, G.; Mora-Sero, I.; Bisquert, J.; Kang, Y. S.; Jacobsson, T. J.; Correa-Baena, J.-P.; Hagfeldt, A. "Properties of Contact and Bulk Impedances in Hybrid Lead Halide Perovskite Solar Cells Including Inductive Loop Elements". The Journal of Physical Chemistry C 2016, 120, 8023-8032.

Guerrero, A.; Marchesi, L. F.; Boix, P. P.; Ruiz-Raga, S.; Ripolles-Sanchis, T.; Garcia-Belmonte, G.; Bisquert, J. "How the Charge-Neutrality Level of Interface States Controls Energy Level Alignment in Cathode Contacts of Organic Bulk-Heterojunction 
Solar Cells". ACS Nano 2012, 6, 3453-3460.

Gunter, P. "Photovoltages, Photocurrents and Photorefractive Effects in Knbo3-Fe". Ferroelectrics 1978, 22, 671-674.

Guo, E.-J.; Xing, J.; Jin, K.-J.; Lu, H.-B.; Wen, J.; Yang, G.-Z. "Photoelectric effects of ultraviolet fast response and high sensitivity in LiNbO3 single crystal". Journal of Applied Physics 2009, 106, 023114.

Guo, R.; You, L.; Zhou, Y.; Shiuh Lim, Z.; Zou, X.; Chen, L.; Ramesh, R.; Wang, J. "Non-volatile memory based on the ferroelectric photovoltaic effect". Nat Commun 2013, 4 .

Hamwi, S.; Meyer, J.; Winkler, T.; Riedl, T.; Kowalsky, W. "p-type doping efficiency of $\mathrm{MoO}_{3}$ in organic hole transport materials". Applied Physics Letters 2009, 94, 253307.

Heyszenau, H. "Electron transport in the bulk photovoltaic effect". Physical Review B 1978, 18, 1586-1592.

Hsiao, Y.-C.; Zang, H.; Ivanov, I.; Xu, T.; Lu, L.; Yu, L.; Hu, B. "Dielectric Interface Effects on Surface Charge Accumulation and Collection towards High-Efficiency Organic Solar Cells". Journal of Applied Physics 2014, 115, 154506.

Hu, Y.; Boudoire, F.; Hermann-Geppert, I.; Bogdanoff, P.; Tsekouras, G.; Mun, B. S.; Fortunato, G.; Graetzel, M.; Braun, A. "Molecular Origin and Electrochemical Influence of Capacitive Surface States on Iron Oxide Photoanodes". The Journal of Physical Chemistry C 2016.

Huang, W.; Nechache, R.; Li, S.; Chaker, M.; Rosei, F. "Electrical and Optical Properties of Transparent Conducting p-Type $\mathrm{SrTiO}_{3}$ Thin Films". Journal of American Ceramic Society 2016, 99, 226-233.

Ichikawa, N.; Arai, M.; Imai, Y.; Hagiwara, K.; Sakama, H.; Azuma, M.; Shimakawa, Y.; Takano, M.; Kotaka, Y.; Yonetani, M.; Fujisawa, H.; Shimizu, M.; Ishikawa, K.; Cho, Y. "Multiferroism at Room Temperature in $\mathrm{BiFeO}_{3} / \mathrm{BiCrO}_{3}(111)$ Artificial Superlattices". Applied Physics Express 2008, 1, 101302.

Ichiki, M.; Furue, H.; Kobayashi, T.; Maeda, R.; Morikawa, Y.; Nakada, T.; Nonaka, K. "Photovoltaic properties of $(\mathrm{Pb}, \mathrm{La})(\mathrm{Zr}, \mathrm{Ti}) \mathrm{O} 3$ films with different crystallographic orientations". Applied Physics Letters 2005, 87, 222903.

Inoue, R.; Takahashi, S.; Kitanaka, Y.; Oguchi, T.; Noguchi, Y.; Miyayama, M. "Enhanced photovoltaic currents in strained Fe-doped LiNbO3 films". physica status solidi (a) 2015, 212, 2968-2974.

Inoue, Y.; Yoshioka, I.; Sato, K. "Polarization effects upon adsorptive and catalytic properties. 1. Carbon monoxide oxidation over palladium deposited on lithium niobate (LiNbO3) ferroelectrics". The Journal of Physical Chemistry 1984, 88, 1148-1151. 
Jalaja, M. A.; Dutta, S. "Ferroelectrics and multiferroics for next generation photovoltaics". Advanced Materials Letter 2015, 6, 6.

Ji, W.; Yao, K.; Liang, Y. C. "Bulk Photovoltaic Effect at Visible Wavelength in Epitaxial Ferroelectric BiFeO3 Thin Films". Advanced Materials 2010, 22, 1763-1766.

Jo, J. Y.; Kim, Y. S.; Noh, T. W.; Yoon, J.-G.; Song, T. K. "Coercive fields in ultrathin BaTiO3 capacitors". Applied Physics Letters 2006, 89, 232909.

Jo, S. B.; Kim, M.; Sin, D. H.; Lee, J.; Kim, H. G.; Ko, H.; Cho, K. "CarrierSelectivity-Dependent Charge Recombination Dynamics in Organic Photovoltaic Cells with a Ferroelectric Blend Interlayer". Advanced Energy Materials 2015, 5, n/a-n/a.

Kakekhani, A.; Ismail-Beigi, S. "Ferroelectric-Based Catalysis: Switchable Surface Chemistry". ACS Catalysis 2015, 5, 4537-4545.

Käppler, W.; Arlt, G. "Photovoltaic effect in pure and modified barium titanate ceramics". physica status solidi (a) 1981, 63, 475-480.

Karger, M.; Schilling, M. "Epitaxial properties of Al-doped $\mathrm{ZnO}$ thin films grown by pulsed laser deposition on $\mathrm{SrTiO}_{3}(001) \ldots$... Physical Review B 2005, 71, 075304.

Khan, M. A.; Nadeem, M. A.; Idriss, H. "Ferroelectric polarization effect on surface chemistry and photo-catalytic activity: A review". Surface Science Reports 2016, 71, 131.

Kholkin, A.; Boiarkine, O.; Setter, N. "Transient photocurrents in lead zirconate titanate thin films". Applied Physics Letters 1998, 72, 130-132.

Kirchartz, T.; Bisquert, J.; Mora-Sero, I.; Garcia-Belmonte, G. "Classification of solar cells according to mechanisms of charge separation and charge collection". Physical Chemistry Chemical Physics 2015, 17, 4007-4014.

Kirchartz, T.; Rau, U. in Advanced Characterization Techniques for Thin Film Solar Cells; Abou-Ras, D., Kirchartz, T., Rau, U., Eds.; Wiley: Berlin, 2011; pp 14.

Kojima, A.; Teshima, K.; Shirai, Y.; Miyasaka, T. "Organometal halide perovskites as visible-light sensitizers for photovoltaic cells". Journal of the American Chemical Society 2009, 131, 6050-6051.

La, N. N.; White, T. P.; Catchpole, K. R. "Optics and Light Trapping for Tandem Solar Cells on Silicon". IEEE Journal of Photovoltaics 2014, 4, 6.

Landsberg, P. T.; Klimpke, C. "Theory of the Schottky barrier solar cell". Proceedings Royal Society (London) A 1977, 354, 101-118.

Lazarev, V. V.; Blinov, L. M.; Yudin, S. G.; Palto, S. P. "Photovoltaic effect in an organic semiconductor controlled by a polymer ferroelectric". Crystallogr. Rep. 2015, 60, 286-288.

Lee, D.; Baek, S. H.; Kim, T. H.; Yoon, J. G.; Folkman, C. M.; Eom, C. B.; Noh, T. W. "Polarity control of carrier injection at ferroelectric/metal interfaces for electrically 
switchable diode and photovoltaic effects". Physical Review B 2011, 84, 125305.

Lee, K. H.; Schwenn, P. E.; Smith, A. R.; Cavaye, H.; Shaw, P. E.; James, M.; Krueger, K. B.; Gentle, I. R.; Meredith, P.; Burn, P. L. "Morphology of All-Solution-Processed "Bilayer" Organic Solar Cells". Advanced Materials 2011, 23, 766-770.

Leguy, A. M. A.; Frost, J. M.; McMahon, A. P.; Sakai, V. G.; Kockelmann, W.; Law, C.; Li, X.; Foglia, F.; Walsh, A.; O/'Regan, B. C.; Nelson, J.; Cabral, J. T.; Barnes, P. R. F. "The dynamics of methylammonium ions in hybrid organic-inorganic perovskite solar cells". Nat Commun 2015, 6, 7124.

Li, G.; Shrotriya, V.; Huang, J.; Yao, Y.; Moriarty, T.; Emery, K.; Yang, Y. "Highefficiency solution processable polymer photovoltaic cells by self-organization of polymer blends". Nature materials 2005, 4, 864-868.

Li, L.; Zhang, Y.; Schultz, A. M.; Liu, X.; Salvador, P. A.; Rohrer, G. S. "Visible light photochemical activity of heterostructured $\mathrm{PbTiO} 3-\mathrm{TiO} 2$ core-shell particles". Catalysis Science \& Technology 2012, 2, 1945-1952.

Lines, M. E.; Glass, A. M. Principles and Applications of Ferroelectrics and Related Materials; Clarendon Press: Oxford, 2001.

Liu, H.; Chen, J.; Ren, Y.; Zhang, L.; Pan, Z.; Fan, L.; Xing, X. "Large Photovoltage and Controllable Photovoltaic Effect in PbTiO3-Bi(Ni2/3+xNb1/3-x)O3- $\delta$ Ferroelectrics". Advanced Electronic Materials 2015, 1, 10.1002/aelm.201400051.

Liu, J.; Zhang, S.; Chen, F.; Yang, C.; Zhou, X. "Measurements and simulation of hysteresis loops of donor-doped strontium bismuth tantalate ceramics". Physics Letters A 2004, 321, 199-204.

Liu, Q.; Khatri, I.; Ishikawa, R.; Fujimori, A.; Ueno, K.; Manabe, K.; Nishino, H.; Shirai, H. "Improved photovoltaic performance of crystalline-Si/organic Schottky junction solar cells using ferroelectric polymers". Applied Physics Letters 2013, 103, 163503.

Liu, X.; Wang, Y.; Burton, J. D.; Tsymbal, E. Y. "Polarization-controlled Ohmic to Schottky transition at a metal/ferroelectric interface". Physical Review B 2013, 88, 165139.

Mai, M.; Ke, S.; Lin, P.; Zeng, X. "Ferroelectric Polymer Thin Films for Organic Electronics". Journal of Nanomaterials 2015, 2015, 14.

Marchioro, A.; Teuscher, J.; Friedrich, D.; Kunst, M.; van de Krol, R.; Moehl, T.; Gratzel, M.; Moser, J.-E. "Unravelling the mechanism of photoinduced charge transfer processes in lead iodide perovskite solar cells". Nat Photon 2014, 8, 250-255.

Masaaki, I.; Yasushi, M.; Takeshi, N. "Electrical Properties of Ferroelectric Lead Lanthanum Zirconate Titanate as an Energy Transducer for Application to Electrostatic- 
Optical Motor". Japanese Journal of Applied Physics 2002, 41, 6993.

Mattoni, A.; Filippetti, A.; Saba, M. I.; Delugas, P. "Methylammonium Rotational Dynamics in Lead Halide Perovskite by Classical Molecular Dynamics: The Role of Temperature". The Journal of Physical Chemistry C 2015, 119, 17421-17428.

Mehta, R. R. "Some theoretical considerations relating to switching and remanence in ferroelectric/photoconductor memory devices". Ferroelectrics 1972, 4, 5-18.

Mehta, R. R.; Silverman, B. D.; Jacobs, J. T. "Depolarization fields in thin ferroelectric films". Journal of Applied Physics 1973, 44, 3379-3385.

Miller, S. L.; Nasby, R. D.; Schwank, J. R.; Rodgers, M. S.; Dressendorfer, P. V. "Device modeling of ferroelectric capacitors". Journal of Applied Physics 1990, 68, 6463-6471.

Miller, S. L.; Schwank, J. R.; Nasby, R. D.; Rodgers, M. S. "Modeling ferroelectric capacitor switching with asymmetric nonperiodic input signals and arbitrary initial conditions". Journal of Applied Physics 1991, 70, 2849-2860.

Misirlioglu, I. B.; Yildiz, M. "Carrier accumulation near electrodes in ferroelectric films due to polarization boundary conditions". Journal of Applied Physics 2014, 116, 024102 .

Mosconi, E.; Quarti, C.; Ivanovska, T.; Ruani, G.; De Angelis, F. "Structural and electronic properties of organo-halide lead perovskites: a combined IR-spectroscopy and ab initio molecular dynamics investigation". Physical Chemistry Chemical Physics 2014, 16, 16137-16144.

Naber, R. C. G.; Asadi, K.; Blom, P. W. M.; de Leeuw, D. M.; de Boer, B. "Organic Nonvolatile Memory Devices Based on Ferroelectricity". Advanced Materials 2010, 22, 933-945.

Nalwa, K. S.; Carr, J. A.; Mahadevapuram, R. C.; Kodali, H. K.; Bose, S.; Chen, Y.; Petrich, J. W.; Ganapathysubramanian, B.; Chaudhary, S. "Enhanced charge separation in organic photovoltaic films doped with ferroelectric dipoles". Energy \& Environmental Science 2012, 5, 7042-7049.

Neaton, J. B.; Ederer, C.; Waghmare, U. V.; Spaldin, N. A.; Rabe, K. M. "Firstprinciples study of spontaneous polarization in multiferroic BiFeO3". Physical Review B 2005, 71, 014113.

Nechache, R.; Harnagea, C.; Carignan, L.-P.; Gautreau, O.; Pintilie, L.; Singh, M. P.; Menard, D.; Fournier, P.; Alexe, M.; Pignolet, A. "Epitaxial thin films of the multiferroic double perovskite $\mathrm{Bi}_{2} \mathrm{FeCrO}_{6}$ grown on (100)-oriented $\mathrm{SrTiO}_{3}$ substrates: Growth, characterization, and optimization". Journal of Applied Physics 2009, 105, 061621.

Nechache, R.; Harnagea, C.; Carignan, L.-P.; Ménard, D.; Pignolet, A. "Epitaxial 
$\mathrm{Bi}_{2} \mathrm{FeCrO}_{6}$ multiferroic thin films". Philosophical Magazine Letters 2007, 87, 231-240.

Nechache, R.; Harnagea, C.; Li, S.; Cardenas, L.; Huang, W.; Chakrabartty, J.; Rosei, F. "Bandgap tuning of multiferroic oxide solar cells". Nature Photonics 2015, 9, 61-67.

Nechache, R.; Harnagea, C.; Licoccia, S.; Traversa, E.; Ruediger, A.; Pignolet, A.; Rosei, F. "Photovoltaic properties of $\mathrm{Bi}_{2} \mathrm{FeCrO}_{6}$ epitaxial thin films". Applied Physics Letters 2011, 98, 202902.

Nechache, R.; Harnagea, C.; Pignolet, A.; Normandin, F.; Veres, T.; Carignan, L.-P.; Ménard, D. "Growth, structure, and properties of epitaxial thin films of first-principles predicted multiferroic $\mathrm{Bi}_{2} \mathrm{FeCrO}_{6}$ ". Applied Physics Letters 2006, 89, 102902.

Nechache, R.; Ruediger, A.; Rosei, F. Combined PN junction and bulk photovolitaic device; patent, U., Ed.; Institut National de la Recherche Scientifique (INRS), Montreal (CA): United States, 2012

Neumark, G. F. "Theory of the Anomalous Photovoltaic Effect of ZnS". Physical Review 1962, 125, 838-845.

Onoda-Yamamuro, N.; Matsuo, T.; Suga, H. "Calorimetric and IR spectroscopic studies of phase transitions in methylammonium trihalogenoplumbates (II) $\dagger$ ". J. Phys. Chem. Solids 1990, 51, 1383-1395.

Paillard, C.; Bai, X.; Infante, I. C.; Guennou, M.; Geneste, G.; Alexe, M.; Kreisel, J.; Dkhil, B. "Photovoltaics with Ferroelectrics: Current Status and Beyond". Advanced Materials 2016, 28, 5153-5168.

Pantel, D.; Alexe, M. "Electroresistance effects in ferroelectric tunnel barriers". Physical Review B 2010, 82, 134105.

Pintilie, L. in Ferroelectrics - Physical Effects; Lallart, M., Ed.; InTech 2011.

Pintilie, L.; Alexe, M. "Metal-ferroelectric-metal heterostructures with Schottky contacts. I. Influence of the ferroelectric properties". Journal of Applied Physics 2005, 98, 124103.

Pintilie, L.; Alexe, M.; Pignolet, A.; Hesse, D. "Bi4Ti3O12 ferroelectric thin film ultraviolet detectors". Applied Physics Letters 1998, 73, 342-344.

Pintilie, L.; Vrejoiu, I.; Hesse, D.; LeRhun, G.; Alexe, M. "Extrinsic contributions to the apparent thickness dependence of the dielectric constant in epitaxial \$lmathrm $\{\mathrm{Pb}\}(\backslash \mathrm{mathrm}\{\mathrm{Zr}\}, \operatorname{Imathrm}\{\mathrm{Ti}\})\{\backslash \operatorname{mathrm}\{\mathrm{O}\}\}_{-}\{3\} \$$ thin films". Physical Review B 2007, 75, 224113.

Pintilie, L.; Vrejoiu, I.; Le Rhun, G.; Alexe, M. "Short-circuit photocurrent in epitaxial lead zirconate-titanate thin films". Journal of Applied Physics 2007, 101, 064109.

Poglitsch, A.; Weber, D. "Dynamic disorder in methylammoniumtrihalogenoplumbates (II) observed by millimeterâ€• wave 
spectroscopy". The Journal of Chemical Physics 1987, 87, 6373-6378.

Preisach, F. "Über die magnetische Nachwirkung. ". Zeitschrift für Physik 1935, 94, 277-302.

Qin, M.; Yao, K.; Liang, Y. C. "Photovoltaic mechanisms in ferroelectric thin films with the effects of the electrodes and interfaces". Applied Physics Letters 2009, 95, 022912.

Qin, M.; Yao, K.; Liang, Y. C.; Shannigrahi, S. "Thickness effects on photoinduced current in ferroelectric $(\mathrm{Pb} 0.97 \mathrm{La0.03})(\mathrm{Zr0.52Ti0.48)O3}$ thin films". Journal of Applied Physics 2007, 101, 014104.

Rastogi, A. C. "Interfacial polymer ferroelectric dipole induced electric field effect on the photovoltaic performance of organic solar cells". Journal of Vacuum Science \&amp; Technology B 2013, 31, 04D112.

Rau, W.; Kron, G.; Werner, J. H. "Rely to Comments on "Electronic transport in dyesensitized nanoporous $\mathrm{TiO} 2$ solar cells- Comparison of electrolyte and solid-state devices"". The Journal of Physical Chemistry B 2003, 107, 13547.

Reece, T. J.; Ducharme, S. "Modeling of metal-ferroelectric-insulator-semiconductor structures based on Langmuir-Blodgett copolymer films". Journal of Applied Physics 2009, 106, 124505.

Ruppel, W.; Von Baltz, R.; Würfel, P. "The Origin of the Photo-Emf in Ferroelectric and Non-Ferroelectric Materials". Ferroelectrics 1982, 43, 109-123.

Sato, H.; Minami, T.; Takata, S.; yamada, Y. Thin Solid Films 1993, 1-2.

Schiff, E. A. "Low-mobility solar cells: a device physics primer with applications to amorphous silicon". Solar Energy Materials and Solar Cells 2003, 78, 567-595.

Scott, J. F.; Kammerdiner, L.; Parris, M.; Traynor, S.; Ottenbacher, V.; Shawabkeh, A.; Oliver, W. F. "Switching Kinetics of Lead Zirconate Titanate Sub-Micron Thin-Film Memories". Journal of Applied Physics 1988, 64, 787-792.

Seidel, J.; Fu, D.; Yang, S.-Y.; Alarcon-Llado, E.; Wu, J.; Ramesh, R.; Ager, J. W. "Efficient Photovoltaic Current Generation at Ferroelectric Domain Walls". Physical Review Letters 2011, 107, 126805.

Sheu, Y. M.; Trugman, S. A.; Park, Y.-S.; Lee, S.; Yi, H. T.; Cheong, S.-W.; Jia, Q. X.; Taylor, A. J.; Prasankumar, R. P. "Ultrafast carrier dynamics and radiative recombination in multiferroic BiFeO3". Applied Physics Letters 2012, 100, 242904.

Shin, K.-S.; Kim, T. Y.; Yoon, G. C.; Gupta, M. K.; Kim, S. K.; Seung, W.; Kim, H.; Kim, S.; Kim, S.; Kim, S.-W. "Ferroelectric Coupling Effect on the Energy-Band Structure of Hybrid Heterojunctions with Self-Organized P(VDF-TrFE) Nanomatrices". Advanced Materials 2014, 26, 5619-5625.

Shockley, W.; Queisser, H. J. "Detailed balance limit of efficiency of p-n junction 
solar cells". Journal of Applied Physics 1961, 32, 510-520.

Smith, R. C. a. J. E. M. “A Unified, Methodology for Modeling Hysteresis in Ferroelectric, Ferromagnetic and Ferroelastic Materials," North Carolina State University,Center for Research in Scientific Computation,Raleigh,NC,27695-8205, 2001.

Starkiewicz, J.; Sosnowski, L.; Simpson, O. "Photovoltaic Effects Exhibited in HighResistance Semi-Conducting Films". Nature 1946, 158, 28-28.

Sturman, B. I.; Fridkin, V. M. Photovoltaic and Photo-refractive Effects in Noncentrosymmetric Materials; CRC Press, 1992.

Suryanarayana, P.; Bhattacharya, K. "Evolution of polarization and space charges in semiconducting ferroelectrics". Journal of Applied Physics 2012, 111, 034109.

Sze, S. M. Physics of Semiconductor Devizes, 2nd ed.; John Wiley and Sons: New York, 1981.

Thakoor, S. "High-Speed Optoelectronic Response from the Edges of LeadZirconate-Titanate Thin-Film Capacitors". Applied Physics Letters 1993, 63, 3233-3235.

Tian, B. B.; Liu, Y.; Chen, L. F.; Wang, J. L.; Sun, S.; Shen, H.; Sun, J. L.; Yuan, G. L.; Fusil, S.; Garcia, V.; Dkhil, B.; Meng, X. J.; Chu, J. H. "Space-charge Effect on Electroresistance in Metal-Ferroelectric-Metal capacitors". Scientific Reports 2015, 5, 18297.

Turrión, M.; Bisquert, J.; Salvador, P. "Flatband potential of F:SnO2 in a TiO2 dyesensitized solar cell: An interference reflection study.". The Journal of Physical Chemistry B 2003, 107, 9397-9403.

Von Baltz, R.; Kraut, W. "Theory of the Bulk Photo-Voltaic Effect in Pure-Crystals". Physical Review B 1981, 23, 5590-5596.

Wang, C.; Jin, K.-j.; Xu, Z.-t.; Wang, L.; Ge, C.; Lu, H.-b.; Guo, H.-z.; He, M.; Yang, G.-z. "Switchable diode effect and ferroelectric resistive switching in epitaxial BiFeO3 thin films". Applied Physics Letters 2011, 98, 192901.

Wang, F.; Rappe, A. M. "First-principles calculation of the bulk photovoltaic effect inKNbO3 and (K,Ba)(Ni,Nb)O3". Physical Review B 2015, 91, 165124.

Wang, F.; Young, S. M.; Zheng, F.; Grinberg, I.; Rappe, A. M. "Substantial bulk photovoltaic effect enhancement via nanolayering". Nat Commun 2016, 7, 10419.

Wang, F. G.; Grinberg, I.; Jiang, L.; Young, S. M.; Davies, P. K.; Rappe, A. M. "Materials Design of Visible-Light Ferroelectric Photovoltaics from First Principles". Ferroelectrics 2015, 483, 1-12.

Wang, F. G.; Grinberg, I.; Rappe, A. M. "Band gap engineering strategy via polarization rotation in perovskite ferroelectrics". Applied Physics Letters 2014, 104.

Weis, R. S.; Gaylord, T. K. "Lithium niobate: Summary of physical properties and 
crystal structure". Applied Physics A 1985, 37, 191-203.

Wong, F.; Perez, G.; Bonilla, M.; Colon-Santana, J. A.; Zhang, X.; Sharma, P.; Gruverman, A.; Dowben, P. A.; Rosa, L. G. "Changing molecular band offsets in polymer blends of (P3HT/P(VDF-TrFE)) poly(3-hexylthiophene) and poly(vinylidene fluoride with trifluoroethylene) due to ferroelectric poling". RSC Advances 2014, 4, 3020-3027.

Wu, W.; Guest, J. R.; Horibe, Y.; Park, S.; Choi, T.; Cheong, S. W.; Bode, M. "Polarization-Modulated Rectification at Ferroelectric Surfaces". Physical Review Letters 2010, 104, 217601.

Wu, Z.; Zhang, Y.; Ma, K.; Cao, Y.; Lin, H.; Jia, Y.; Chen, J.; Li, H. "Strong visiblelight photovoltaic effect in multiferroic $\mathrm{Pb}(\mathrm{Fe} 1 / 2 \mathrm{~V} 1 / 2) \mathrm{O} 3$ bulk ceramics". physica status solidi (RRL) - Rapid Research Letters 2014, 8, 36-39.

Würfel, P. Physics of Solar Cells. From Principles to New Concepts; Wiley: Weinheim, 2005.

Yang, B.; Yuan, Y.; Sharma, P.; Poddar, S.; Korlacki, R.; Ducharme, S.; Gruverman, A.; Saraf, R.; Huang, J. "Tuning the Energy Level Offset between Donor and Acceptor with Ferroelectric Dipole Layers for Increased Efficiency in Bilayer Organic Photovoltaic Cells". Advanced Materials 2012, 24, 1455-1460.

Yang, F.; Zhou, Y. C.; Tang, M. H.; Liu, F. "Simulation of electric displacement hysteresis and strain butterfly loops in perovskite ferroelectric films". Journal of Applied Physics 2009, 106, 014110.

Yang, S. Y.; Martin, L. W.; Byrnes, S. J.; Conry, T. E.; Basu, S. R.; Paran, D.; Reichertz, L.; Ihlefeld, J.; Adamo, C.; Melville, A.; Chu, Y.-H.; Yang, C.-H.; Musfeldt, J. L.; Schlom, D. G.; Ager, J. W.; Ramesh, R. "Photovoltaic effects in BiFeO3". Applied Physics Letters 2009, 95, 062909.

Yang, S. Y.; Seidel, J.; Byrnes, S. J.; Shafer, P.; Yang, C.-H.; Rossell, M. D.; Yu, P.; Chu, Y.-H.; Scott, J. F.; Ager, J. W.; Martin, L. W.; Ramesh, R. "Above-bandgap voltages from ferroelectric photovoltaic devices". Nature Nanotechnology 2010, 5, 143.

Yang, W.; Yu, Y.; Starr, M. B.; Yin, X.; Li, Z.; Kvit, A.; Wang, S.; Zhao, P.; Wang, $\mathrm{X}$. "Ferroelectric Polarization-Enhanced Photoelectrochemical Water Splitting in TiO2BaTiO3 Core-Shell Nanowire Photoanodes". Nano Letters 2015, 15, 7574-7580.

Yang, Y.; Xu, W.; Xu, X.; Wang, Y.; Yuan, G.; Wang, Y.; Liu, Z. "The enhanced photocurrent of epitaxial $\mathrm{BiFeO} 3$ film at $130{ }^{\circ} \mathrm{C} "$. Journal of Applied Physics 2016, 119 , 044102.

Yang, Y. S.; Lee, S. J.; Yi, S.; Chae, B. G.; Lee, S. H.; Joo, H. J.; Jang, M. S. "Schottky barrier effects in the photocurrent of sol-gel derived lead zirconate titanate thin film capacitors". Applied Physics Letters 2000, 76, 774-776. 
Yi, H. T.; Choi, T.; Choi, S. G.; Oh, Y. S.; Cheong, S. W. "Mechanism of the Switchable Photovoltaic Effect in Ferroelectric BiFeO3". Advanced Materials 2011, 23, 3403-3407.

Young, S. M.; Rappe, A. M. "First Principles Calculation of the Shift Current Photovoltaic Effect in Ferroelectrics". Physical Review Letters 2012, 109, 116601.

Young, S. M.; Zheng, F.; Rappe, A. M. "First-Principles Calculation of the Bulk Photovoltaic Effect in Bismuth Ferrite". Physical Review Letters 2012, 109.

Young, S. M.; Zheng, F.; Rappe, A. M. "First-Principles Materials Design of HighPerforming Bulk Photovoltaics with the LiNbO3 Structure". Physical Review Applied 2015, 4 .

Yuan, G.-L.; Wang, J. "Evidences for the depletion region induced by the polarization of ferroelectric semiconductors". Applied Physics Letters 2009, 95, 252904.

Yuan, Y.; Reece, T. J.; Sharma, P.; Poddar, S.; Ducharme, S.; Gruverman, A.; Yang, Y.; Huang, J. "Efficiency enhancement in organic solar cells with ferroelectric polymers". Nature materials 2011, 10, 296-302.

Yuan, Y.; Xiao, Z.; Yang, B.; Huang, J. "Arising applications of ferroelectric materials in photovoltaic devices". Journal of Materials Chemistry A 2014, 2, 60276041.

Yun, Y.; Altman, E. I. "Using Ferroelectric Poling to Change Adsorption on Oxide Surfaces". Journal of the American Chemical Society 2007, 129, 15684-15689.

Zenkevich, A.; Matveyev, Y.; Maksimova, K.; Gaynutdinov, R.; Tolstikhina, A.; Fridkin, V. "Giant bulk photovoltaic effect in thin ferroelectric BaTiO3 films". Physical Review B 2014, 90, 161409.

Zhai, X. Z.; Deng, H. M.; Zhou, W. L.; Yang, P. X.; Chu, J. H.; Zheng, Z. "Structural, optical and magnetic tunability in KBiFe2O5 multiferroics". RSC Advances 2015, 5, 82351-82356.

Zhang, G. H.; Wu, H.; Li, G. B.; Huang, Q. Z.; Yang, C. Y.; Huang, F. Q.; Liao, F. H.; Lin, J. H. "New high Tc multiferroics KBiFe2O5 with narrow band gap and promising photovoltaic effect (vol 3, 1265, 2013)". Scientific Reports 2013, 3.

Zhang, J.; Su, X.; Shen, M.; Dai, Z.; Zhang, L.; He, X.; Cheng, W.; Cao, M.; Zou, G. "Enlarging photovoltaic effect: combination of classic photoelectric and ferroelectric photovoltaic effects". Scientific Reports 2013, 3, 2109.

Zhang, Z.; González, R.; Díaz, G.; Rosa, L. G.; Ketsman, I.; Zhang, X.; Sharma, P.; Gruverman, A.; Dowben, P. A. "Polarization Mediated Chemistry on Ferroelectric Polymer Surfaces". The Journal of Physical Chemistry C 2011, 115, 13041-13046.

Zheng, F.; Takenaka, H.; Wang, F.; Koocher, N. Z.; Rappe, A. M. "First-Principles Calculation of the Bulk Photovoltaic Effect in $\mathrm{CH} 3 \mathrm{NH} 3 \mathrm{PbI} 3$ and $\mathrm{CH} 3 \mathrm{NH} 3 \mathrm{PbI}-\mathrm{xClx} "$. 
The Journal of Physical Chemistry Letters 2015, 6, 31-37.

Zheng, F.; Xu, J.; Fang, L.; Shen, M.; Wu, X. "Separation of the Schottky barrier and polarization effects on the photocurrent of $\mathrm{Pt}$ sandwiched $\mathrm{Pb}(\mathrm{Zr} 0.20 \mathrm{Ti0}$.80)O3 films". Applied Physics Letters 2008, 93, 172101.

Zhou, Y.; Fang, L.; You, L.; Ren, P.; Wang, L.; Wang, J. "Photovoltaic property of domain engineered epitaxial BiFeO3 films". Applied Physics Letters 2014, 105, 252903.

Zhuravlev, M. Y.; Sabirianov, R. F.; Jaswal, S. S.; Tsymbal, E. Y. "Giant Electroresistance in Ferroelectric Tunnel Junctions". Physical Review Letters 2005, 94, 246802. 